\title{
Phyllotaxis: Some progress, but a story far from over.
}

\author{
Matthew F. Pennybacker ${ }^{a, *}$, Patrick D. Shipman ${ }^{\mathrm{b}}$, Alan C. Newell ${ }^{\mathrm{c}}$ \\ ${ }^{a}$ University of New Mexico, Albuquerque, New Mexico 87131 USA \\ ${ }^{b}$ Colorado State University, Fort Collins, Colorado 80523 USA \\ ${ }^{c}$ University of Arizona, Tucson, Arizona 85721 USA
}

\begin{abstract}
This is a review article with a point of view. We summarize the long history of the subject and recent advances and suggest that almost all features of the architecture of shoot apical meristems can be captured by pattern-forming systems which model the biochemistry and biophysics of those regions on plants.
\end{abstract}

Keywords: Phyllotaxis, Pattern Formation

\section{Contents}

\begin{tabular}{ll|l}
1 & Introduction & 1
\end{tabular}

1.1 History of the study of phyllotaxis . . . . . . . . . . . . . . . . . . . . . . . 5

1.2 The phyllotactic lattice and dual lattice . . . . . . . . . . . . . . . . . . . . . . . . . . 7

1.3 Kinematics of phyllotactic pattern formation . . . . . . . . . . . . . . . . . . . . . 9

1.4 Phyllotactic transitions . . . . . . . . . . . . . . . . . . . . . . . . 10

2 Explanations for Phyllotactic Patterns 11

2.1 Categories of explanations . . . . . . . . . . . . . . . . . . . . . . . . 11

2.2 Teleological explanations . . . . . . . . . . . . . . . . . . . . . . . . . . . . . 12

2.3 Biochemical and biophysical mechanisms . . . . . . . . . . . . . . . . . . . . . 15

2.4 Mathematical models . . . . . . . . . . . . . . . . . . . . . . . . . . . 17

3 Analysis and Simulations 21

3.1 Simulations: What we do, what we measure . . . . . . . . . . . . . . . . . . . . . . . 23

3.2 The results of the simulations: Fibonacci patterns . . . . . . . . . . . . . . . . . . . . 25

3.3 Derivation of the amplitude or order parameter equations . . . . . . . . . . . . . . . . . . . 28

3.4 Results from the amplitude equation analysis . . . . . . . . . . . . . . . . . . . . . . 32

3.5 Transitions and whorls . . . . . . . . . . . . . . . . . . . . . . . 34

\begin{tabular}{|l|l|}
\hline Conclusions & 35 \\
\hline
\end{tabular}

\section{Introduction}

Observe the seeds of sunflowers, bracts on pine cones, spines on cacti, leaves on foliage plants, and analogous structures (leaf homologues) found in pictures throughout this article. In many of these examples, such as on the sunflower head of Figure 1 and the pine cone and cactus of Figures 2a and 2b the leaf homologues are arranged in spirals. Strikingly, the spiral pattern on the cactus of Figure $2 \mathrm{~b}$ is almost identical to that on the pine cone of Figure $2 \mathrm{a}$. In both cases, the homologues lie at the intersections of two families of spirals, and the numbers of spirals in these families (89 and 55 at the outer edges of the sunflower of Figure 1 and 13 and 8 on the pine cone and cactus of Figures 2a and 2b are successive members of the

\footnotetext{
${ }^{*}$ Corresponding author

Email address: pennybacker@math.unm.edu (Matthew F. Pennybacker)
} 
Fibonacci sequence. In contrast, on the cactus of Figure 2c, the succulent of Figure 2d, and many other plants, the spines or leaf homologues are arranged in opposite pairs that alternate in angle, the so-called decussate or 2-whorl arrangement. Three-whorl patterns, in which triplets of homologues separated by $120^{\circ}$ from each other, and for which the following and preceding triplets are rotated by $60^{\circ}$, are also common (see Figure 10a).

The arrangement (taxis) of leaves (singular: phyllo, plural: phylla) or their analogs on plants is referred to as phyllotaxis. Phyllotaxis has intrigued natural scientists for ages, served as a tool for identifying and classifying plants, motivated questions on optimal packing, and provided challenges and clues to understanding the biochemistry and biomechanics of plant growth. It is surprising that, despite much attention over the years, only recently have quantitative explanations emerged for the wonderful architecture seen near the shoot apical meristems (SAM's) of plants. The goal of this review is to tell the story to date and to provide substantial evidence for the idea that plants and other organisms can pursue optimal strategies by employing naturally occurring patterns driven by instabilities initiated by biochemical and biomechanical processes. Moreover, the length scales associated with the driving mechanisms are not microscopic and connected in any obvious way with genetic instructions but are macroscopic and of the same order as the phenomena observed.

While this article is a review, it adopts, over the telling of a story, a point of view: That all the mysteries and challenges of phyllotaxis will ultimately be explained by the behaviors of instability-generated patterns in auxin and stress fields in the neighborhood of plant SAM's. We provide substantial evidence that points to this conclusion.

To this end, it is good idea to provide the reader with a roadmap spelling out what it is we do in each section and why. The introductory Section 1.1 introduces the reader to some of the landmarks in the history of the subject. A more extensive history of the study of phyllotaxis may be found in [1]. This section also introduces important terminologies used as descriptive tools such as, for example, the notions of parastichies and the fact that the polar coordinates of the phylla lie on cylindrical lattices. These lattices can be characterized by one of two measures. The first arises from an appropriate and 'obvious to the eye' choice of basis vectors for the lattice from which two important further quantities, the rise and divergence angle, can be calculated. Due to plant growth, the rise and divergence angle evolve, the latter often tending to the golden angle in the outer reaches of the meristem. This evolution is captured by a diagram due to Van Iterson which is central to all attempts to explain the observed phyllotactic configurations. But there is also an equally important second set of measures, dual coordinates, associated with the normals to the preferred choice of basis vectors for the lattice. In Section 1.2, we introduce both sets of phyllotactic coordinates and outline why it is that the dual coordinates, which are basically Fourier modes, are more useful in explanations which are based on mechanistic rather than teleological models. In Section 1.3 , we discuss the kinematics of phyllotactic pattern formation, namely how the incipient phylla, called primordia, are initiated in a generative annulus in the neighborhood of the shoot apical meristems (SAM's) of plants. Here we learn that the radial position $R$ of the generative annulus changes as the plant grows. In many cases, it increases, but in some cases, such as during the seed formation stage of sunflowers, it decreases. The upshot is that the nature of the pattern, whether it is has a spiral or whorl structure, may change depending on the radius of the generative annulus at which primordia first form. This necessitates a discussion, which we begin in Section 1.4 between spiral patterns with different numbers of spirals in their spiral families or between spiral patterns and whorl patterns. Sometimes the transitions are smooth; sometimes they involve defect formation. The main ideas are discussed in Section 1.4 and we provide conclusions about transitions from our models in Section 3.5

Section 2 discusses in detail the main two approaches to date which purport to explain phyllotaxis. The first approach is teleological in that it posits rules manifested as cellular automaton algorithms which are based on the plant placing its phylla according to some optimization principle. Some rules have an observational footing. They are based on repeated and careful experiments by the noted botanist Hofmeister, who encoded the outcome of his observations in a set of rules, now named after him, which we list in Section 1.1. Some readers may prefer the use of the word "phenomenological" to the word "teleological" due to the observational motivation of the rules for these discrete models. We, like D'Arcy Thompson, prefer the latter because these rules, while consistent with observation, express the fact that primordia are located in positions designed to achieve some desirable outcome. We cover, in Sections 2.1 and 2.2, the original ideas of Van Iterson and discuss how they connect with the Hofmeister rules and the more modern pioneering 
works of Douady and Couder, and Atela, Golé and Hotton. The reason we spend some time including them in the review is the remarkable result, which may have ramifications for many biological organisms, that the teleological and mechanistic approaches, albeit starting from very different premises, have stunningly similar outcomes. In Section 2.3 , we describe the basis for our mathematical models which we detail in Section 2.4 The dominant physics and chemistry operable in plant meristems, recently reported in series of seminal experiments by the teams of Reinhardt, Kuhlemeier, Meyerowitz and Traas, and contained in earlier insights of Paul Green and colleagues, govern what the mathematical models should be. The key results stem from the observed facts that phyllotactic patterns are naturally produced by instabilities, connected to both the distribution of the growth hormone auxin and to the local stress-strain fields. Moreover, we show that the partial differential equations which naturally arise from modeling the actions of the fundamental players in the plant's meristem are familiar from general studies on pattern formation. If the auxin instability dominates, then the partial differential equation (pde) governing the auxin fluctuation field $u(x, y, t)$ is essentially of Swift-Hohenberg type

$$
u_{t}=-\left(\nabla^{2}+1\right)^{2} u+\mu u-\beta \nabla(u \nabla u)-u^{3} .
$$

The reader should think of $u(x, y, t)$ as representing the difference between the local auxin concentration and its spatial average. The solution $u=0$ then corresponds to a state of uniform auxin concentration in what we introduce as the generative annulus in Section 1.3. The generative annulus, made from newly formed material, lies between the the region in which a patterned state already exists and the central region of slowly growing cells. The uniform auxin concentration solution $u=0$ is unstable and will be invaded by a front traveling from the patterned region. In forming the developing pattern, the quadratic term in (1) plays a central role in choosing the pattern structure, similar to its role in planar geometries, which leads to the dominance of hexagonal (honeycomb) patterns in many situations. In order that the various Fourier modes which combine to shape this structure travel synchronously, so that patterns are locally preserved, it is important that the parameters are in a range where the invading front is pushed rather than pulled, concepts that we explain in Section 3.2.

In Section 3 , we analyze the patterns naturally produced by a traveling front of this pde and show how they answer many of the key questions and challenges which arise in modeling phyllotaxis. In Section 3.1, we outline the main ideas behind the method used for simulating the model pde (1), discuss what it is that we measure and indicate how the scheme can be readily adapted to more general surfaces of revolution. In Section 3.2, we present the main results. Key among them are the following: (i) If one Fourier analyzes the field $u(r, \theta)$ in angle, namely the solution of (1) when the pattern is complete, we find that the only modes significantly generated by nonlinear interactions are those whose circumferential wavenumbers belong to Fibonacci sequences. (ii) The amplitudes and wavevectors of the dominant modes at any generative radius $R$ follow universal curves. (iii) The patterns are self-similar so that the amplitudes and the squares of the radial wavenumbers of the dominant modes are the same at $R$ and $R \phi$, where $\phi$ is the golden number. (iv) The configuration of phylla resulting from the mechanistic models follow closely the van Iterson diagram resulting from the discrete models.

Because the simulations lead to fields $u(r, \theta)$ which are best understood by the behaviors of their Fourier modes, in Section 3.3 we develop a center manifold approach which recasts the pde (1) as a set of odes for the dominant mode amplitudes. The amplitude equations are extremely useful in helping to understand the behaviors observed in results (i)-(iv). They are also much richer than the amplitude equations used for describing hexagonal and roll patterns in planar geometries because the dominant modes in the phyllotactic context change with the generative radius $R$. In Section 3.2, we show how the amplitude equations reproduce the results of direct simulations. Finally, in Section 3.5. we discuss how transitions occur and relate our findings with the language of phase transitions used in condensed matter physics.

Our main conclusions from Section 3 are the following:

1. Almost all features of phyllotaxis can be reproduced by models which are based on auxin and stressstrain distributions in the vicinity of the shoot apical meristems of plants. The models lead to partial differential equations of pattern-forming type whose solutions consist of quasiperiodic shapes dominated by a preferred wavenumber $k_{0}$. As the patterns invade the unstable regions of uniform auxin distribution in the form of pushed fronts, they leave behind arrays of maxima at which new phylla are created. These arrangements in most cases consist of either Fibonacci progressions or whorls. A Fibonacci progression is a global pattern of spirals which smoothly evolves in radius so that the num- 
bers in the spiral families increase or decrease through a Fibonacci sequence. At certain radii $R$, the arrangements may be described as hexagonal tessellations; in between these radii, they are rhombic. The patterns are robust in the sense that they do not change qualitatively and do change smoothly under changes of the parameters $\mu$ and $\beta$ in some open set.

2. An unexpected, but welcome albeit surprising, outcome is that the phyllotactic arrangements agree with those arising as fixed points from dynamical system algorithms which reflect optimal packing and least crowded space criteria. Thus the mechanistic and teleological approaches to phyllotaxis are seen to be compatible. Of course, the former gives more information because it includes field rather than isolated point behaviors. Nevertheless, the compatibility of the two approaches, one based on plain old biochemistry and biophysics, the other on optimal packing ideas, opens up the exciting possibility that pattern-forming pdes may provide an additional avenue of attack for formidable packing challenges and also suggest that nature, in many contexts, may use pattern forming systems to pursue and achieve optimal outcomes. We repeat for emphasis: The surprising fact that the models based on physical and biochemical mechanisms produce results consistent with models based on principles of optimal packing may, and in fact should, have broad ramifications in many natural contexts. The advantages can be cut both ways. In some cases, it may be valuable to replace the complexity of the pattern evolution governed by a pde to a more simple set of discrete rules if one's principal aim is to map the configuration of field maxima.

3. While it is gratifying that the teleological and mechanistic approaches yield consistent results, there is a distinct advantage to the latter approach when it comes to comes to explaining the differences between the configurations of phylla and the surface morphologies of plants. Although the model which treats the coupled system (Equations $(22)$ of Section 2.4) is not analyzed in this review, the results have been previously detailed in 44. There, we show how it is possible that the auxin fluctuation distribution field which determines the location of the phylla can differ from the surface deformation field which determines surface shape. This difference is apparent, for example, in saguaro and barrel cacti, which are strongly ribbed. The mechanistic models also make testable predictions. For example, we predict that the pattern front laying down sunflower seeds will travel with a nearly constant average speed with small variations (see Fig. 27) such that the speed through the hexagonal regions is slightly faster than the speed through the rhombic regions, and that the front speed at each $R$ is the same as that at $R \phi$, where $\phi$ is the golden number. This can, in principle, be experimentally checked and lead to confidence in the model and help to estimate model parameters.

4. Fibonacci progressions are preferred because, as we explain in Sections 3.3 and 3.4, a. quadratic nonlinearities in the pattern forming equations mean that new modes and shapes are produced by the vector additions of wave vectors of the dominant Fourier modes, b. the patterns must continuously evolve as the magnitude of the radius $R$ of the generating annulus where new phylla are made changes, c. the only solutions which can change continuously are those dominated by a developing sequence of wavevectors created by binary combinations which obey the Fibonacci rule; the Fibonacci addition gives rise to wave vectors which are drawn towards the preferred wavenumber $k_{0}$ circle; all other binary combinations give rise to wave vectors which are pushed away and the result is their amplitudes are small compared to those of the modes created by Fibonacci addition.

5. In Section 3.5. we show that whorls exist for finite intervals in the generative annulus radius parameter $R$. For low $R$, these intervals may overlap, as between 2 and 3 whorls, but as $R$ increases they are separate. Therefore, it is not easy for a growing plant to follow a whorl progression. Nevertheless, decussates or 2-whorls and 3-whorls are widely observed. We suggest the two reasons for this are that (a) many plants have an ingrown bias (the cotyledon) which makes the decussate state easily accessible and (b) that at the SAM's of some plants, notably growth tips at the ends of twigs and branches, the generative annulus radius $R$ remains more-or-less constant over much of the plant's growth. In such instances, we might expect to see decussate structures, or $(2,3,5)$ spirals, or 3 -whorls repeated along the branch.

6. Fibonacci progressions are long-lived states which arise when pattern forming systems have preferred length scales and the symmetries of rotation and translation but do not have the field reversal symmetry (so that quadratic nonlinearities are important) and where the pattern forms as a pushed front annulus by annulus. Thus they should be observable in many contexts. 
In the final section, Section 4, we summarize the main results, list some open and exciting questions and challenges, and suggest other contexts in which Fibonacci patterns may be important.

This review contains both a compilation of our previous works [39, 40, 41, 42, 44, 46, 51, 52, 53, as well as new results $(e . g$. the nature of transitions, the remarkably broad self-similar properties of the field $u(r, \theta)$, and ramifications for center manifold theory). We hope that the reader will by the end be convinced of three things. The first is that optimal outcomes can be achieved by following the basic mechanistic rules of physics and chemistry. The second is that, while progress has been made, there are many fascinating challenges in phyllotaxis still open for the curious mind to explore. The story is far from over. The third is that while careful experiments are crucial to continued progress, it does not require elaborate experiments for ordinary folk to enjoy the wonderful architectures seen near the meristems of plants. All it takes is a mountain hike or a walk through a garden and a keen eye for observation of the plants in nature's finest laboratories.

\subsection{History of the study of phyllotaxis}

Phyllotactic patterns have practical utility to botanists: That leaves have regular arrangements on plants was noted by Theophrastus (371-287 BC) in his influential book Enquiry into Plants. The encyclopedia of Pliny the Elder, entitled Natural History and published circa 77-79 AD, categorizes plants using detailed descriptions of their phyllotactic patterns. Phyllotactic observation as a part of botanical classification was firmly established by the Swiss naturalist Charles Bonnet (1720-1793) in his book sur l'Usage des Feuilles dans les Plantes.

The patterns have also captivated natural scientists for their simple beauty and mathematical intrigue. Leonardo da Vinci expressed fascination with phyllotactic patterns. Kepler noted a curious mathematical property of spiral phyllotactic patterns, which the reader can discover too by counting the numbers of counterclockwise (13) or clockwise (8) spirals in the spiral families of the pine cone and cactus in Figures 2a and 2b and the sunflower of Figure 1. The numbers of spirals in these examples, and in most plants that exhibit spiral phyllotaxis are consecutive members of the regular Fibonacci sequence $F_{n}: 1,1,2,3,5,8,13,21, \ldots$ If the spiral counts do not belong to the regular Fibonacci sequence, they are most likely consecutive members of the double Fibonacci sequence $2,2,4,6,10,16,42, \ldots$ or the Lucas sequence $1,3,4,7,11,18,29, \ldots$ According to one account [27, of the plants with spiral phyllotaxis, $91 \%$ have counts in the regular Fibonacci sequence, $5 \%$ in the double Fibonacci sequence, and $2 \%$ in the Lucas sequence.

Schimper in 1830 introduced the term parastichy pair to refer to the numbers of spirals in the clockwise and counterclockwise spiral families of spiral phyllotaxis. Kepler's observation in this terminology is that the parastichy pair of a typical spiral phyllotactic pattern is $\left(F_{n}, F_{n+1}\right)$ for integers in the regular Fibonacci sequence $F_{n}$. The observation of the Fibonacci sequence in spiral phyllotaxis is complemented by another parameter introduced by Schimper: If one assigns polar coordinate values $(r, \theta)$ to the center of each leaf homologue in spiral phyllotaxis and orders the coordinate pairs in a sequence $\left(r_{n}, \theta_{n}\right)$ according to increasing values of the radial coordinate $r$, then the angle between consecutively ordered angles is constant; $\theta_{n+1}-\theta_{n}=$ $2 \pi d(\bmod 2 \pi)$ for some divergence angle $2 \pi d$. The sequence $F_{n}$ and the golden ratio $\phi=\frac{1}{2}(1+\sqrt{5})$ are intimately related in that the ratios $F_{n+1} / F_{n}$ approach $\phi$ for increasing $n$, and for regular Fibonacci phyllotaxis, $d$ is approximately $\frac{1}{2}(3-\sqrt{5})=1-1 / \phi$.

The radial coordinates also obey regularity in a manner that we describe in Section 1.2. A commonly observed notion of regularity is that of the plastochrone ratio which is the ratio $r_{n+1} / r_{n}$ for those plants for which this ratio is independent of $n$. The plastochrone ratio applies to those plants for which primordia of equal area are produced at shoot apical meristem, and subsequent growth of the stem moves the developing primordia exponentially away from the apex. In this case, primordia (developing into phylla) that are further from the shoot apical meristem will be larger in size. In contrast, the seeds of the sunflower head of Figure 1 are nearly constant in area except near the center of the seed head. The observations of constant divergence angle and radial regularity as described in Section 1.2 will mean that the $\left(r_{n}, \theta_{n}\right)$ coordinates lie, for large $r$, on a point lattice, namely all integer combinations of two basis vectors. A lattice means that the points, representing the centers of the leaf homologues, lie on straight lines in the chosen coordinate system. Such a lattice is also called a Bravais lattice, after Auguste and Louis Bravais, crystalographer and botanist siblings, who represented phyllotactic patterns using a point lattice on a cylinder and gave precise definitions to parastichy pairs, the divergence angle, and the plastochrone ratio.

If an image of a pattern of spiral phyllotaxis were to be projected to the $(r, \theta)$-plane and Fourier analyzed at each value of the radius, then the parastichy pattern numbers at any radius would correspond to the 
circumferential $(\theta)$ wavenumbers of the two dominant Fourier modes at that radius. All phyllotactic coordinates would be determined from this information. One of the main challenges of phyllotaxis is to explain why it is that instead of the Fourier transform being supported on the integers, it is supported mainly on subsets which are generated by the Fibonacci rule.

The phyllotactic patterns that we have described as Bravais lattices are the product of a process of formation of primordia, small bumps that develop into leaves or leaf homologues at the plant tip, and subsequent outward (in the reference frame of the apex) movement of the developing primordia as the shoot grows. After years of careful observation of plant apices, Hofmeister [23] proposed in the 1800's a set of simple geometric and kinematic rules governing this process. These rules, as quoted from the formulation of Douady and Couder [9], read

1. The stem apex is axisymmetric.

2. The primordia form at the periphery of the apex (Region 2 in Figure 12), and, due to the shoot's growth, they move away from the center with a radial velocity $V(r)$ which may depend on their radial location.

3. New primordia are formed at regular times intervals (the plastochrone time $T$ ).

4. The incipient primordium forms in the largest available space left by the previous ones; see Figure 3d.

5. Outside of a region of radius $R$ there is no further reorganization leading to changes in the angular position of the primordium.

Snow and Snow [55, 56 extended Hofmeister's rules to account for primordia that form simultaneously at the same radius. Under the Hofmeister rules, the only allowed phyllotactic configurations would be spiral. The relaxation to the Snow and Snow criterion allowed for whorl structures to compete with Fibonacci spiral structures.

The Hofmeister observation that primordia appear in the most open space available is a geometric one, but also one that has a reasonable botanical interpretation: such a rule would seem to lead to an optimal packing of leaves. Airy provided in 1873 an experimental apparatus that explained phyllotaxis in terms of economy of space. In this experiment, Airy glued spheres on alternating sides of a stretched elastic band and proceeded to relax the band. In the resulting configuration, the spheres pack into a arrangement such that their centers geometrically lay on a cylinder. In fact, the centers of the spheres lay on cylindrical Fibonacci-spiral phyllotactic lattices.

Motivated in part by Airy's work, the Dutch botanist Gerrit van Iterson (1878-1972) developed a mathematical approach to phyllotaxis. In his 1907 book Mathematische und mikroskopisch-anatomische Studien ueber Blattstellungen (Mathematical and Microscopic-Anatomical Studies of Phyllotaxis), Van Iterson represented a plant as a cylinder, and phyllotactic elements as circular disks when that cylinder is rolled onto the plane (see Figure 4). In Van Iterson's approach, the problem of phyllotaxis thus became the study of "regular arrays of circles on a cylindrical surface." These regular arrays consisted of nonoverlapping packings of circles with centers positioned on a Bravais lattice, and in which each disk is tangent to its four nearest neighbors. Varying the diameter of the disks relative to that of the cylinder (see Figure 5), Van Iterson found what divergence angles and parastichy pairs are possible for a given value of this parameter, as depicted in the Van Iterson diagram of Figure 6. We will work in the paper with a parameter which we call the rise $\rho$. This is the vertical distance between successive packing disk centers and is related to the ratio of the packing disk radius $r_{p}$ and the cylinder radius which is taken to be such that the cylinder circumference is unity. For small $r_{p}, \rho$ scales as $r_{p}^{2}$. If the disk circumference had been taken to be unity and the cylinder radius $R$ been allowed increase instead, $\rho$ would scale, for large $R$, as $1 / R^{2}$. In the packing diagram Figure $5, \rho$ is the vertical distance between the centers of the disks marked 1 and 2 or between those marked 2 and 3.

A hexagonal packing of disks only occurs at certain discrete ratios of disk radius to cylindrical circumference; two of these are marked on Figure 5. At all ratios in between these values, the geometric pattern is rhombic. The observation that one can only achieve hexagonal packing at certain discrete ratios of disk to cylinder radius is crucial since a hexagonal packing is optimal. Referring to Figure 5 at most locations, as the radius evolves between two hexagonal states, the packing is less hexagonal and more rhombic and thus less than optimal when seen from the planar viewpoint. It is this feature, the fact that hexagons will not fit at at all ratios of disk radius to cylindrical circumference, that leads to the composite hexagonal-rhombic or Fibonacci spiral patterns. Contrary to packing an infinite two-dimensional plane, a uniform pattern of 
hexagons is not preferred in the cylindrical geometry, although, as is illustrated by Figure 5, as the ratio decreases, the composite pattern is a continuous transition from one hexagonal pattern to another through rhombic structures. We note that the Van Iterson construction will allow either the 5 -disk to descend through $(2,3)$ or the 4 -disk to descend through $(1,3)$. This leads to the diagram in Figure 6 in which both branches from $(1,2,3)$ to $(1,3,4)$ and $(2,3,5)$ are equally allowed. We will find later, however, when the algorithms become slightly more complicated, that the former branch is less favored. Nevertheless, the Van Iterson diagram, modified, is a central pillar in understanding phyllotactic configurations, and we will be returning to it time and time again.

The notion that phyllotactic patterns, or at least those that display Fibonacci spirals, achieve an optimal packing suggests that such patterns may arise from minimization of some energy function. Indeed, Levitov [32, 33. showed in 1991 that phyllotactic patterns and Fibonacci sequences can arise in layered superconductors that minimize a global interaction energy.

Energy minimization also plays a central role in an ingenious experimental device built by Douady and Couder [8, 9] in 1992. In Douady and Couder's experiments, a small dome in the center of a plate represents the plant apex. The plate is placed in a vertical magnetic field that is stronger at the edges of the plate than in the middle, and ferromagnetic drops (representing primordia) are periodically dropped onto the center of the central dome. The drops fall to the bottom of the central dome and then move radially outward, following the gradient of increasing magnetic strength. The magnetic field is chosen so that the velocity $V(r)$ is an exponentially increasing function of the radius. After choosing a radial direction in which to fall from the top of the central dome, the drops do not change their angular coordinate. How the drops initially choose their angular coordinate is of interest, and here the central point is that the drops form repelling magnetic dipoles. A drop that falls on the central dome moves to the position on the boundary of the dome as determined by the repulsions of the drops that recently formed and are moving away from the center, and the experimental result is that there is a constant divergence angle between the angular coordinates of successively-dropped drops. Denoting the radius of the central dome by $L$, the initial speed of the drops after falling to the boundary of the dome by $V_{0}=V(L)$, and the time period in which drops are dropped onto the central dome by $T$, the plastochrone ratio of the resulting pattern is $G \doteq \frac{V_{0} T}{L}$. $G$ is a parameter chosen by the experimenter. As Douady and Couder decreased $G$, the resulting divergence angle approaches the golden angle, and the pattern appears as one with a Fibonacci parastichy pair. The suggestion of this experiment is that simple dynamical rules as proposed by Hofmeister are at the center of the phyllotactic process. In subsequent numerical simulations, Douady and Couder [10, 11, following a modification of Hofmeister's rules proposed by Snow and Snow [55, 56, replaced the $T$-periodic formation of primordia with a new parameter-the space needed for a new primoridium to form. The parameter $G=\frac{V_{0} T}{L}$ was replaced by a parameter $\Gamma=\frac{d_{0}}{L}$, where $d_{0}$ is the diameter of a newly formed primordium; Douady and Couder refer to $\Gamma$ as Van Iterson's parameter. It is an analog of the rise $\rho$ that we use in this paper. This approach allowed for the formation of whorled as well as spiral patterns.

As demonstrated by the experiments and related numerical simulations of Douady and Couder, simple dynamical rules may reproduce many phyllotactic patterns. In Section 2 , we describe a much simpler system designed by Atela, Gole, and Hotton [2, 24] which is also based on the ideas of Hofmeister and Douady and Couder. These results are not, however, connected to biochemical or biomechanical mechanisms or parameters.

\subsection{The phyllotactic lattice and dual lattice}

The three left illustrations of Figure 5 picture the packing formations along the $(1,2,3)$ and $(2,3,5)$ branches of the Van Iterson diagram. The domain is a cylinder of circumference $2 \pi R$. The upper left panel shows a hexagonal lattice with disk 0 touching disks 1,2 , and 3 . The middle left panel shows a rhombic packing with disk 0 touching disks 2 and 3 but not disk 1 . A lattice is obtained if we replace the disks by their centers, as shown in Figure 8 for the upper- and middle-left panels of Figure 5 . The vertical distance between successively numbered points we define to be $\lambda=2 \pi R \rho$, where $\rho$ is the rise in the van Iterson diagram (see Figure 6). In Section 1.1, we defined the plastochrone ratio for those plants for which the ratio $r_{n+1} / r_{n}$ of radial coordinates is constant. For such plants, if one interprets the vertical axis of Figure 8 as $s=\ln (r)$, then the plastochrone ratio is the vertical distance $\lambda=2 \pi R \rho$ between successively numbered points. The circumferential distance $\sigma$ between two points is $2 \pi R d$, where $2 \pi d$ is the divergence angle. Thus, if $s=r$, an increase in $R$ as from the top to the bottom panel of Figure 8 will result in a constant primordium 
area (which may be interpreted as the area of a Voronoi cell in a Voronoi tessellation corresponding to the lattice) only if $\rho$ scales like $1 / R^{2}$, or equivalently $\lambda$ scales like $1 / R$. It is this choice of $s=r$ and scaling of $\rho$ that lead to phyllotactic configurations of constant primordium area in the disk. The sunflower seed head of Figure 1 is a good example of such a planform, except near the center where the seeds are smaller.

The coordinates $(s, \sigma)$ of the points marked $0,1,2,3$ in Figure 8 are respectively $\boldsymbol{\omega}_{0}=(0,0), \boldsymbol{\omega}_{1}=$ $2 \pi R(\rho, d), \boldsymbol{\omega}_{2}=2 \pi R(2 \rho, 2 d-1), \boldsymbol{\omega}_{3}=2 \pi R(3 \rho, 3 d-1)$. One can readily see that all points $0,1,2,3,4,5,6,7,8, \ldots$ lie on a straight line in the extended picture or on the cylinder when the lines $\sigma= \pm \pi R$ are identified. Any position $\boldsymbol{\omega}_{j}$, may be written as an integer linear combination of the basis vectors $\boldsymbol{\omega}_{1}$ and $\boldsymbol{u}_{0}=(0,2 \pi R)$. For example, $\boldsymbol{\omega}_{2}=2 \boldsymbol{\omega}_{1}-\boldsymbol{u}_{0}, \boldsymbol{\omega}_{3}=3 \boldsymbol{\omega}_{1}-\boldsymbol{u}_{0}, \boldsymbol{\omega}_{4}=4 \boldsymbol{\omega}_{1}-2 \boldsymbol{u}_{0}, \ldots$

If one looks at the middle left panel of Figure 5 or the analogous lattice in the bottom panel of Figure 8 , we observe two things. First, the most natural basis is the one for which the lengths $\boldsymbol{\omega}_{j}$ are shortest, here $\boldsymbol{\omega}_{2}$ and $\boldsymbol{\omega}_{3}$. Secondly, the lengths $\left|\boldsymbol{\omega}_{2}\right|$ and $\left|\boldsymbol{\omega}_{3}\right|$ are equal and the positions of $\boldsymbol{\omega}_{2}$ and $\boldsymbol{\omega}_{3}$ are opposed-that is, they lie on opposite sides of the vertical line drawn through 0 . Both of these properties, nearest neighbors equidistant and opposed are also properties of the fixed-point lattices generated by the discrete algorithms of Atela, Gole, and Hotton as well as Douady and Couder which place each new point in a configuration according to some "most open space" principle. The equidistant property of the two nearest neighbors

$$
\boldsymbol{\omega}_{m}=2 \pi R(m \rho, m d-q), \boldsymbol{\omega}_{n}=2 \pi R(n \rho, n d-p),
$$

where $p$ and $q$ are integers chosen so that $0 \leq m d-q, n d-p<1$ (most often given by $q=2 m-n, p=n-m$, the two previous integers in the Fibonacci sequence) gives us the branch of the Van Iterson diagram joining the hexagonal configurations $n-m, m, n$ to $m, n, m+n$ for all $m, n$ in the coordinates $\rho, d$. The locus of these points is a circle

$$
\rho^{2}+\left(d-\frac{p n-q m}{n^{2}-m^{2}}\right)^{2}=\frac{(p n-q m)^{2}}{\left(n^{2}-m^{2}\right)^{2}}+\frac{q^{2}-p^{2}}{n^{2}-m^{2}}
$$

whose center is $\rho=0, d=\frac{p n-q m}{n^{2}-m^{2}} \rightarrow 1-1 / \phi=1 / \phi^{2}$ as $m, n \rightarrow \infty, \lim \frac{n}{m}=\phi$. The divergence angle converges to the golden divergence angle $2 \pi d=2 \pi(1-1 / \phi)$.

We have alluded to two sets of explanations for phyllotactic behaviors. One posits a set of rules and uses discrete algorithms or minimum energy principles to construct point configurations which are the suggested locations of emerging phylla. The second is mechanistic and involves understanding the behavior of certain fields, such as the concentration of the plant hormone auxin or the stress field in the plant's tunica. For the remainder of this section, we take $s=r$; analogous expressions result from replacing $r$ by functions $s(r)$. These fields $w(r, \theta)$ will be quasiperiodic functions of the position coordinates $r, \theta$ on the plant surface near its SAM and can be represented as linear combinations of Fourier modes $\sum_{m} a_{m}(r) e^{i\left(\phi_{m}(r)+m \theta\right)}+$ c.c., where the sum is over the positive integers $m$. The local radial wavenumber is the derivative $l_{m}=\phi_{m}^{\prime}(r)$. In many cases, it will turn out that the circumferential wavenumbers $m$ for which the real-valued mode amplitudes $a_{m}(r)$ are, at any given radius $r$, dominant, are subsets of the integers $\left\{m_{j}\right\}_{j=1}^{N}$ belonging to Fibonacci sequences. This, together with the assumption that the local radial wavenumber varies slowly with $r$, allows us to approximate the local field $w(r, \theta)$ (we use $w$ for a general field such as auxin concentration or stress, and reserve $u$ to connote auxin concentration fluctuation) as the combination

$$
w(r, \theta)=\sum_{j=1}^{N} a_{j} \cos \left(l_{j} r+m_{j} \theta-\phi_{j}\right) .
$$

The radial and circumferential dependence of the phases can be approximated as $l_{j} r-m_{j} \theta-\phi_{j}$ being constant. The sum (4) may be written as

$$
w(r, \theta)=\sum a_{j} \cos \left(l_{j} r+\frac{m_{j}}{r} \sigma(r)-\phi_{j}\right),
$$

where $\sigma(r)=r \theta$. In contrast of the wavevectors $\left(l_{j}, m_{j}\right)$ of $(4)$, the components of the wavevectors $\left(l_{j}, m_{j} / r\right)$ of (5) have the same dimensions (of inverse length). Therefore, we shall henceforth write the wavevector as $\left(l_{j}, m_{j} / r\right)$ and refer to $\left(l_{j}, m_{j}\right)$ as the wavenumber pair. The total wavenumber, referred to in the introduction, is $l_{j}^{2}+m_{j}^{2} / r^{2}$. 
The maxima (or minima) of fields (4) are point configurations which are defined by lattices constructed by the intersection of lines of constant phase $l_{j} r+m_{j} \theta-\phi_{j}=2 \pi p_{j}, p_{j}$ an integer, corresponding to those integers $p_{j}$ for which the amplitudes $a_{j}$ are dominant. They turn out to be lattices in $(r, \theta)$-coordinates. These lines of constant phase are precisely the lines defining the lattice discussed in the disk packing of the cylinder. The middle left panel in Figure 5 illustrates a lattice for which we can use the basis $\boldsymbol{\omega}_{2}, \boldsymbol{\omega}_{3}$. But we can also use a basis of lines; namely, we can describe the lattice not in terms of the positions $\boldsymbol{\omega}_{j}$ of the lattice points, but by the normals of the line joining $\boldsymbol{\omega}_{0}$ to each $\boldsymbol{\omega}_{j}$ or the wavenumber pair $\left(l_{j}, m_{j}\right)$. This basis is much more useful when we analyze the second class of explanations based on physical and biochemical mechanisms. The second basis is, of course, completely equivalent to the first and is called the dual basis. At the radius $r=R$, the lattice has parastichies $m, n$ with basis vectors (2) The dual basis, suitably normed, is

$$
\boldsymbol{k}_{m}=\frac{1}{R}\left(\frac{1}{\rho}(q-m d), m\right), \boldsymbol{k}_{n}=\frac{1}{R}\left(\frac{1}{\rho}(p-n d), n\right) .
$$

The radial wavenumber is $l_{m}=\frac{1}{R \rho}(q-m d)$. We use these relations in Section 3 to compare the results of simulations of the pde (1) to the Van Iterson diagram. For now, we note that, since $\left|\boldsymbol{\omega}_{m}\right|=\left|\boldsymbol{\omega}_{n}\right|$, the dominant wavevectors $\boldsymbol{k}_{m}$ and $\boldsymbol{k}_{n}$ also have equal lengths, namely $\sqrt{l_{m}^{2}+m^{2} / R^{2}}$ and $\sqrt{l_{n}^{2}+n^{2} / R^{2}}$. In Section 3. we shall also see that, just as $d$ and $\rho$ follow the branches in the Van Iterson diagram, so also will the dominant wave vectors follow universal paths in $(l, m / R)$-space.

In [51, 53, we give a detailed derivation of the expressions for the lattice basis vectors (2) and the dual basis vectors (6) in terms of the phyllotactic parameters. The derivation in these references includes more general lattices for which more than one point may appear at each radius.

\subsection{Kinematics of phyllotactic pattern formation}

New material added at regions of active cell growth and division called meristems lead to growth of a shoot or root or formation of flowers. A meristem at the tip (apex) of a plant shoot where new leaves or flowers form is called a shoot apical meristem (SAM). Those SAMs that lead to flowers are called floral meristems (or inflorescence meristems on the sunflower, since the capitulum is actually an inflorescence composed of many flowers). Meristems at root tips are called root meristems. Relevant for phyllotaxis are shoot apical meristems, including floral meristems and inflorescence meristems. Figure 9 shows the inflorescence meristem of a sunflower and shoot apical meristems of cacti.

The SAM at the tip of a cactus is illustrated in Figure 10. The outer layer of the plant, the tunica, is stiffer than the inner layers, the corpus. Both the tunica and the corpus grow during plant growth, but growth occurs differently in different regions of the growth tip. Hernández, et. al. [22] provide a description of the growth behavior of epidermal cells in the flower of Anagallis. Figure 11 shows electron microscope images of the forming flowers on which individual cells are outlined. The growth of the outlined cells was evaluated via a procedure developed by Goodall and Green [15. Figure 11 shows outlines of cells on the same developing flower, with Figure $11 \mathrm{~b}$ recorded at a slightly later time than Figure11a. Each cell is viewed as a polygon that changes shape and size slightly between recordings. The major directions of growth and the growth in these directions are measured and recorded as a "rate cross" placed in the middle of each polygon in the figures; the same cross is placed in the middle of the corresponding cells in the two figures. A longer line in a cross denotes a larger growth in the line's direction; a circle denotes a cell that has not really grown. Notice that there is little growth in the middle of the developing flower, and more, largely radial, growth at the outer edges.

These and similar studies [13] lead to thinking of the SAM as having three zones as depicted in the schematic representation of Figure 12 . The SAM consists of Regions 1, 2, and 4 in the diagram. The tunica region of the SAM (Regions 1 and 2) is distinguished from the corpus (Region 4) in that tunica cells divide primarily in a plane perpendicular to the plant, whereas corpus cells divide in any direction [22, 49. The tunica Regions 1 and 2 are distinguished from each other in that cells of Region 1 grow slowly and divide infrequently, whereas cells of Region 2 grow relatively quickly and divide more frequently.

It is in the Region 2 that phylla form as bumps (or localized regions of oriented cell division; see Section 2.3 called primordia; thus, we will call Region 2 the generative region. After their formation, these primordia develop further in shape so that they become, for example, leaves or florets; that is, they become phylla. As the plant grows, the newly formed phylla move out into what we label as Region 3. In Region 3, 
the phylla mature and move out radially but do not change their angular positions. Meanwhile, new material emanating from the inner part of the SAM becomes the new generative annulus, the new Region 2, and the new region for primordium formation.

The average radius $R$ of the annular generative region may change over time. In the case of the seedhead of the sunflower, $R$ decreases as the front, which is the generative annulus for the emerging seeds or florets, propagates from the outside to the inside. For cacti or pinecones, $R$ increases as the plant tip increases in width. In either case, as $R$ changes, the phyllotactic pattern that forms changes as well. The pattern undergoes transitions.

The reason that the pattern has to change when the radius of the generative annulus changes is that the average spacing of primordia is an intrinsically chosen length depending on plant characteristics. It is the preferred wavelength in our mechanistic models. Therefore, as $R$ changes, the number of bumps which will fit around the annulus must change as well.

\subsection{Phyllotactic transitions}

Bifurcations (phase changes) induced by parameter changes in pattern-forming systems can be of either discontinuous or continuous nature. First-order (Type I) phase changes are typical. In this case, the pattern makes a discontinuous jump from one state to another as some stress parameter $(e . g$. the vertical temperature gradient in a horizontal layer of convecting fluid, the applied stress to a beam, the generative radius $R$ in the present context) increases. Less common are second-order (Type II) phase changes, in which the pattern changes continuously with the parameter. Plants exhibit both Type I and Type II phase transitions as the parameter $R$ varies. Type I transitions are typically associated with whorl patterns, whereas Type II transitions occur in spiral patterns.

To illustrate a Type II transition, we depict in Figure 13 a central result of this review, a numerical simulation of (1) on a disk that models the formation of a sunflower seedhead. This and similar simulations are explained in detail in Section 3 , but we show the result here to illustrate the nature of a Type II transition. The initial condition for the simulation is an induced pattern only at the outer boundary of the disk. This induced pattern models the result of the phyllotactic pattern that has already been established in the leaves surrounding the inflorescence meristem and is a Fibonacci-spiral pattern with parastichy pair $(34,55)$. The pattern propagates inwards towards the center of the disk, and the result at a time for which the front has propagated nearly to the center of the disk is shown in Figure 13

Although there are no defects, the pattern does change with decreasing radius $r$. Inspect the regions labelled as regions A, B, and C, which respectively have mean radial coordinates $r=75 / \phi, r=75 / \sqrt{\phi}$, and $r=75$, where $\phi$ is the golden number. One notes that the lattice is nearly hexagonal in regions $\mathrm{A}$ and $\mathrm{C}$, but is rhombic in region $\mathrm{B}$. The hexagonal and rhombic structures become more apparent upon transforming the three regions as in panels A, B, and C of Figure 13 . Denoting the mean radius of a region by $\hat{r}$, the vertical axis in the transformed panels is $r-\hat{r}$, and the horizontal axis is $\theta$. Counting the numbers of counterclockwise and clockwise spirals, the parastichy pair of the pattern are observed to be $(34,55)$ in the annular region including region $\mathrm{C}$, whereas the parastichy pair is $(21,34)$ in the annular region that includes region $\mathrm{A}$.

The sequence illustrated in Figure 13 achieves a transition between parastichy pairs and from hexagons to squares back to hexagons, continuously without the introduction of defects. This is thus an example of a Type II transition. The functions $u(r, \theta)$ plotted in Figure 13 are the results of numerical simulations, and in Section 3 we find the decomposition into Fourier modes, so that, as described in Section 1.2,

$$
u(r, \theta)=\sum a_{j} \cos \left(l_{j} r+m_{j} \theta-\phi_{j}\right)
$$

As the pattern front propagates to the center of the disk, the radius at which the pattern is being formed decreases. That this is a Type II transition is evident in that the amplitudes $a_{j}$ in (7) are continuous functions of the radius $r$. In the simulation, the pattern is locally a hexagonal lattice at $r=75$, and the three largest amplitudes are those of three modes with wavevectors $\boldsymbol{k}_{21}, \boldsymbol{k}_{34}, \boldsymbol{k}_{55}$ of equal length. A hexagonal lattice also occurs at $r=75 / \phi$, and at this radius, the three largest amplitudes are those of the three modes with wavevectors $\boldsymbol{k}_{13}, \boldsymbol{k}_{21}, \boldsymbol{k}_{34}$. The pattern has transitioned in dominant wavevectors (those with the largest corresponding amplitudes) from $r=75$ to $r=75 / \phi$. However, the circumferential wavenumbers of two of the modes with the largest amplitudes are the same at these values of $r$, and the radial wavenumbers of these modes change continuously with $r$. We note this by classifying this as a (II,2) transition-a transition of Type II under which two of the dominant wavevectors are preserved. 
The (II,2) transition is one example of an array of transitions that are observed in plants. In general, we classify phyllotactic transitions by two indices, the first of which is I or II and denotes whether the transition is of Type I or II. For the second index, we note that, as illustrated in the discussion of Figure 13 (and described in more detail in 52]), the three Fourier modes of the pattern with the largest amplitudes have corresponding wavevectors $\boldsymbol{k}_{m}, \boldsymbol{k}_{n}, \boldsymbol{k}_{m+n}$ satisfying $\boldsymbol{k}_{m}+\boldsymbol{k}_{n}=\boldsymbol{k}_{m+n}$. We refer to the circumferential wavenumbers corresponding to these three dominant amplitudes as the parastichy triple $(m, n, m+n)$. We could introduce the second index in one of two ways. The first way (perhaps we might refer to this as the botanist's definition) is the number of circumferential wavenumbers shared by the before and after states. But, there is a possible subtle point. In making the definition this way, we would be assuming that the corresponding radial wavenumbers change continuously. Sometimes, as we show in Figure 38 of Section 3.5. the radial wavenumber of one of the shared indices may change sign. Thus, we have a preference for a revised definition which uses the second index to connote the number of shared wavenumber pairs, namely shared radial and circumferential wave numbers.

We conclude by illustrating examples of Type I (discontinuous) transitions. In contrast to the Type II transitions typical of sunflowers, Type I transitions are common in whorl phyllotaxis in which a group of $g$ phylla form at the same radius $r$ of the apex. The transition may occur directly, so that a whorl with parastichy triple $(m, n, m+n)=(g, g, 2 g)$ transitions to a whorl with parastichy triple $(m, n, m+n)=$ $(g+1, g+1,2 g+2)$ [30. Such a transition preserves none of the numbers in the parastichy triple, so we refer to it as a Type $(\mathrm{I}, 0)$ transition. Figure 14 shows transverse sections of a shoot apical meristem undergoing a Type $(\mathrm{I}, 0)$ transition.

Alternatively, an increase in whorl number may occur through two steps mediated by spiral phyllotaxis: the parastichy undergoes the transitions $(m, m, 2 m) \rightarrow(m, m+1,2 m+1) \rightarrow(m+1, m+1,2 m+2)$. Since one parastichy number is preserved in each transition, we refer to this as a Type (I,1) transition. An example of a Type $(\mathrm{I}, 1)$ transition in an Argentinian saguaro is shown in Figure 15.

A Type I transition may preserve two of the parastichy numbers in a parastichy triple. The sequence in Figure 16 shows stages in a transition from a $(3,5,8)$ pattern to a $(5,8,13)$ pattern. This contrasts with the continuous Type (II,2) transitions in that the transition is mediated by defects. We refer to such a transition as a transition of Type $(I, 2)$.

\section{Explanations for Phyllotactic Patterns}

\subsection{Categories of explanations}

There are two categories of explanations for phyllotaxis. The first we call teleological. It has the form "X is so in order that Y holds;" e.g., Tigers have stripes in order to achieve better camouflage. Such explanations are manifested in rules which express the idea that each new primordium is initially placed according to a least crowded, or optimal packing criterion, which would presumably give it the best access to light and nutrients. Now, the presence of stripes may indeed give the species of tiger which develops them a competitive advantage over rivals on evolutionary time scales, but that is not directly why they come to be. Tiger stripes arise because of competition between morphogens in the skin, which in some circumstances lead to the preference of one morphogen over another in neighboring regions. Likewise, the epidermal ridges on the tips of our fingers, on palms, and on soles, while giving fingers the advantage of better feel, are there because, very early on in the life of the fetus, shrinking volar pads give rise to compressive stresses and wrinking in the enveloping epidermal layer 29]. Plants do not have on-board computers to make such decisions on the positioning of their phylla. So, how is it that the plants achieve such outcomes?

To answer this question, we turn to the second category of explanations, the "hows," the mechanistic and self-organizational. Instead of positing a set of rules, we seek to understand the physics, mechanics, and biochemistry of the plant's tunica and corpus in the neighborhood of the shoot apical meristem (SAM) and the effects which result from biochemically and mechanically induced instabilities in that vicinity. In these explanations, the incipient phylla or primorida are sited at the maxima of certain fields, either those of the growth hormone auxin or of stress fields, and their positioning is determined by the configurations of field maxima of patterns generated by these instabilities.

And here is one of the remarkable conclusions of our recent work: It turns out that the configurations and outcomes achieved by both categories of explanations are the same. The outcome of the mechanistic approach is richer. It potentially gives us information about fields and addresses the connections between 
phyllotactic configurations and surface morphologies. But, the configuration of initiating sites turns out to be the same. This remarkable finding leads to an extraordinarily exciting possibility: that nature may use instability-generated patterns to produce optimal strategies in a variety of organisms.

It is worth ending this subsection with words illustrating the prescience of two great men with regards to the conclusion stated above. D'Arcy Thompson was a mathematician, classicist, and zoologist and pioneer in promoting the role of mechanism and self organization in nature. Rudyard Kipling was a poet and masterful story teller with vivid imagination. Both men had Irish connections.

But the use of the teleological principle is but one way, not the whole or the only way, by which we may seek to learn how things came to be, and to take their places in the harmonious complexity of the world. To seek not for the ends but for the antecedents is the way of the physicist, who finds 'causes' in what he has learned to recognize as fundamental properties, or inseparable concomitants, or unchanging laws, or matter and of energy. In Aristotle's parable, the builders have laid one stone upon another. It is as a mechanism, or a mechanical construction, that the physicist looks upon the world; and Democritis, first of physicists and one of the greats of the Greeks, chose to refer all natural phenomena to the mechanisms and set the final cause aside.

Still, all the while, like warp and woof, mechanics and teleology are interwoven together, and we must not cleave to the one nor despise the other; for their union is rooted in the very untrue of totality. We may grow shy or weary of looking to a final cause for an explanation of our phenomena; but after we have accounted for these on the plainest principles of mechanical causation, it may be useful and appropriate to see how the final cause would tally with the other, and lead towards the same conclusions. In our own day, the philosopher neither minimises nor unduly magnifies the mechanical aspect of the the Cosmos; nor need the naturalist either exaggerate or belittle the mechanical phenomenon which are profoundly associated with Life, and inseparable from our understanding of Growth and Form.

D'Arcy Thompson, On Growth and Form

For the Colonel's lady an' Judy O'Grady

Are sisters under their skins!

Rudyard Kipling, The Ladies

\subsection{Teleological explanations}

We divide the teleological explanation of phyllotaxis again into two categories: static models and dynamic models. In each category, phylla are represented as points on a cylinder or a disk. Static models consider the plant only after it is fully grown and predict the selected phyllotactic arrangement by optimizing a lattice of phylla with respect to some quantity, such as packing efficiency, contact pressure or entropy. Dynamic models capture the time evolution of the arrangement by specifying some rule for laying down phylla individually so as to locally optimize a similar quantity. Arrangements predicted by static models often manifest as long-time behavior of dynamic models in which the repeated application of a discrete rule leads to a fixed point configuration predicted by the static model. However, dynamic models tend to consider primarily the relaxation to these fixed points and only briefly consider how the pattern of points laid down changes continuously as some key parameter changes slowly in space-time.

The purpose of this section is to outline some of the key ideas in the static and dynamic models, to recount some of the history and to discuss their predictions. The discussion here is an extension (it now contains the argument as to why parts of certain branches in the Van Iterson diagram are not accessed) of that originally given in [46]. 


\subsubsection{Static models}

One of the first mathematical approaches to the problem of phyllotaxis was due to Van Iterson [58. We have already discussed some of his main ideas. Here we revisit and expand on that discussion. In particular, we will focus on the key difference in Figure 5 between the sequence of packings where disk 5 descends between disks 2 and 3 and that sequence where disk 4 descends between disks 1 and 3 . The difference is key to understanding the fact that, in the dynamic models we discuss in the next subsection, certain segments of the branches in the Van Iterson diagram have gaps. We will also reconsider the diagram from the point of view of the dual, or wavevector, coordinates, and thereby forge a connection with the results that emerge from the mechanistic explanations.

Recall that Van Iterson's idea was that leaves on a stem could be approximated by identical circular disks on the surface of a cylinder, with no overlapping, so that their centers form a regular lattice. The density of a particular lattice may be quantified by the packing efficiency $\eta$, defined to be the proportion of the surface of the cylinder covered by disks with the maximum possible radius. The lattice selected by the plant would be the one which gives the most efficient packing. He also observed that we need only to consider simple lattices, in which no two points lie at the same axial position. A cylindrical lattice that is not simple may be split into an integer number of identical lattices that are simple.

By 1911, it had been known for over a century that a close hexagonal packing is densest among regular arrangements on the plane, but Van Iterson encountered the following problem: simple hexagonal lattices are not always possible on the cylinder. Illustrating this fact is the middle left panel in Figure 5. If we take the vector separating the points labelled 0 and 1 , it is not difficult to see that the entire lattice is generated by integer multiples of this vector, wrapping around the cylinder as needed. This implies that each simple lattice must have a single generative spiral running through every lattice point, with consecutive lattice points separated by a constant axial distance $\rho$ and angle $2 \pi d$. Choosing either of these quantities arbitrarily, in general, results in a lattice that is not hexagonal. In fact, there are only countably many $\rho$ and $2 \pi d$ that yield hexagonal lattices. Furthermore, if we index the points consecutively along the generative spiral of a simple hexagonal lattice, for instance, the indices of the nearest neighbors must be related as $(m, n, m+n)$ with $\operatorname{gcd}(m, n)=1$. We choose $m<n$ by convention. The two lattices on the top and bottom left of Figure 5 are illustrations of simple hexagonal lattices. Notice that the hexagonal lattice is not perfectly aligned with the axis of the cylinder. The top left figure has a right-tilted hexagon. The bottom-left hexagon is left-tilted.

We define the term $n$-parastichy to be the family of spirals winding through every $n$-th point in a lattice. For instance, in either lattice of Figure 5, the 2-parastichy consists of a spiral through the centers of disks 0 and 2 and another parallel spiral through the centers of disks 1,3 , and 5 . It is possible to draw an $n$-parastichy for any $n$ by connecting lattice points whose indices differ by $n$. On the other hand, there are only two or three parastichies that are most visible. These are the ones formed by connecting each point with its nearest neighbors. As we have noted, it is customary to refer to a lattice by its two most visible parastichies, given by the two nearest neighbors or each point. In the top left lattice of Figure 5 the most visible parastichies are exactly the neighbors 1,2 and 3 , so we may refer to this as a $(1,2),(1,3),(2,3)$, or $(1,2,3)$ lattice. In general, when the lattice is not hexagonal, the parastichy numbers of the lattice will be uniquely defined.

Of course, our goal is to understand the variety of patterns observed on plants. The true value of $\rho$ is related to the growth rate of the plant and the time between the formation of new primordia, both of which vary between different plant species, different specimens of the same plant species, and even over the lifetime of a single specimen. It is certainly not restricted to values that guarantee the existence of a hexagonal lattice, so we must investigate how to maintain a dense packing between hexagonal states. Consider the situation illustrated in Figure 5. If we think of the transition from one hexagonal packing to the next as an axial compression, resulting from a decrease of $\rho$, then we can imagine the $(m, n)=(1,2)$ to $(m, n)=(2,3)$ transition as the disk centered at point 5 slipping between disks 2 and 3 to touch disk 0 . We could also imagine disk 4 slipping between disks 1 and 3 to form $(m, n)=(1,3)$ illustrated by the right panels in Figure 5 These correspond to transitions in parastichy numbers $(1,2) \rightarrow(2,3) \rightarrow(3,5)$ and $(1,2) \rightarrow(1,3) \rightarrow(3,4)$ respectively. In general, Van Iterson, who was much influenced by ideas of Schwendener [50], showed that any $(m, n)$ hexagonal lattice can compress symmetrically to either $(m, m+n)$ or $(n, m+n)$ and that during the transition the densest lattice is rhombic, in that the two nearest neighbors remain equidistant. The set of all possible rhombic cylindrical lattices is illustrated in Figure6 6ach bifurcation point in this tree diagram 
corresponds to a hexagonal lattice, for which each point is preceded by three equidistant nearest neighbors, and the two branches in decreasing $\rho$ correspond to the two transition possibilities described above. Each segment connecting the bifurcation points corresponds to a continuum of rhombic lattices, each having the same parastichy numbers. Note that the choice of divergence angle $2 \pi d$ for a fixed rise $\rho$ is not unique. Different Fibonacci-like sequences lead to different divergence angles, with different angular differences $2 \pi d$ between successive lattice points.

We note that in both cases, the $123 \rightarrow 235$ and $123 \rightarrow 134$ transitions, the lattices remain rhombic. Namely, since in the first transition, disks 0 and 2 and disks 0 and 3 remain in contact, the distances from 0 to 2 (0 to 3), which we denote $\left|\boldsymbol{\omega}_{2}\right|$ (respectively $\left|\boldsymbol{\omega}_{3}\right|$ ), remain equal. Indeed, if $\boldsymbol{\omega}_{2}, \boldsymbol{\omega}_{3}$, and $\boldsymbol{\omega}_{5}$ are the vectors joining the centers of disk 0 to the centers of disks 2,3 , and 5 , then $\boldsymbol{\omega}_{2}+\boldsymbol{\omega}_{3}=\boldsymbol{\omega}_{5}$. The corresponding relation in the dual wavevector space, where $\boldsymbol{k}_{2}=\left(l_{2}, \frac{m_{2}}{R}\right), \boldsymbol{k}_{3}=\left(l_{3}, \frac{m_{3}}{R}\right)$, and $\boldsymbol{k}_{5}=\left(l_{5}, \frac{m_{5}}{R}\right)$, are the perpendicular directions to the lines $\boldsymbol{\omega}_{2}, \boldsymbol{\omega}_{3}, \boldsymbol{\omega}_{5}$ if $\boldsymbol{k}_{2}+\boldsymbol{k}_{3}=\boldsymbol{k}_{5}$. As either $R$, the radius of the cylinder, increases, or $r$, the disk radius, decreases, the legs formed by $\boldsymbol{\omega}_{2}, \boldsymbol{\omega}_{3}$ spread, and center of disk 5 is drawn in until all four disks $0,2,3,5$ touch. The equidistance condition $\left|\boldsymbol{\omega}_{2}\right|=\left|\boldsymbol{\omega}_{3}\right|$ means that also, throughout the transitions, $\left|\boldsymbol{k}_{2}\right|=\left|\boldsymbol{k}_{3}\right|$. By inspection, one can see that, for the top left panel, $\boldsymbol{k}_{1}$ (the wave vector perpendicular to $\boldsymbol{\omega}_{1}$, the vector joining the centers of disk 0 and disk 1), $\boldsymbol{k}_{2}$ and $\boldsymbol{k}_{3}$ are equal, and $\boldsymbol{k}_{1}$ and $\boldsymbol{k}_{3}$ have negative values of $l_{1}$ and $l_{3}$, whereas $l_{2}$ is positive. The wave vector $\boldsymbol{k}_{3}$, the resolvent of $\boldsymbol{k}_{1}$ and $\boldsymbol{k}_{2}\left(\boldsymbol{k}_{1}+\boldsymbol{k}_{2}=\boldsymbol{k}_{3}\right)$, has equal length. As $R$ increases ( $r$ decreases), $\boldsymbol{k}_{2}$ and $\boldsymbol{k}_{3}$ are equal in length, their radial components become more positive (respectively negative), and $\boldsymbol{k}_{5}=\boldsymbol{k}_{2}+\boldsymbol{k}_{3}$ gets drawn towards the circle $\left|\boldsymbol{k}_{2}\right|=\left|\boldsymbol{k}_{3}\right|=l_{2}^{2}+\frac{m_{2}^{2}}{R^{2}}=l_{3}^{2}+\frac{m_{3}^{2}}{R^{2}}=$ constant until the bottom left panel is reached. Note that the new hexagon has a left tilt, whereas the starting hexagon had a right tilt.

To see the motion of the wavevectors, look at Figure 26, which is the result of direct simulation on a disk of radius $R \approx 233$. It shows the $(5,8,13)$ to $(8,13,21)$ transition between $R \approx 13$ and 21 but applies equally well to how the dual coordinates in the static model vary with the rise $\rho$. The parameter $R$ is then inversely proportional to $\rho$. Note as $R$ increases, both the wavevectors $\boldsymbol{k}_{8}$ and $\boldsymbol{k}_{13}$ move along the critical circle $\left|\boldsymbol{k}_{8}\right|=\left|\boldsymbol{k}_{13}\right|=$ constant, the former down and right, the latter down and left. The resolvent $\boldsymbol{k}_{21}=\boldsymbol{k}_{8}+\boldsymbol{k}_{13}$ is thereby drawn in. On the other hand, the wavevector created by the irregular Fibonacci addition, $\boldsymbol{k}_{5}+\boldsymbol{k}_{13}$ gets pushed away from the critical circle. The vector $\boldsymbol{k}_{5}=\boldsymbol{k}_{13}-\boldsymbol{k}_{8}$, which played a central role for smaller values of $R$, gets pushed away from the critical circle. As we shall see later, the critical circle represents the optimal choice of wavevector length to minimize a certain energy functional which arises in the biochemically driven model which describes the onset of phyllotaxis.

The situation in the $(1,2,3)$ to $(1,3,4)$ transition is significantly different. In this case, the vectors $\boldsymbol{\omega}_{1}$ and $\boldsymbol{\omega}_{3}$ of the parastichies both point to the right. Their corresponding wavevectors $\boldsymbol{k}_{1}$ and $\boldsymbol{k}_{3}$ point left, namely their radial wavenumbers $l_{1}$ and $l_{3}$ are both negative. In the transition, as $R$ decreases (or $r$ decreases), disk 3 must move left and cross the vertical line through 0 . The corresponding wave vector has to move from the left to the right side of the circle $\left|\boldsymbol{k}_{1}\right|=\left|\boldsymbol{k}_{3}\right|=$ constant. In other words, for a certain interval of $R$ (or $r$ ), disks 1 and 3 are not opposed, namely they are not on the opposite sides of the vertical line through the center of disk 0 . Once that value of $R$ at which the center of disk 3 moves to the left of this vertical line, the vertices $\boldsymbol{\omega}_{1}$ and $\boldsymbol{\omega}_{3}$ are opposed (point to the right and left of the vertical line). Once this happens, the wavevectors $\boldsymbol{k}_{1}$ and $\boldsymbol{k}_{3}$ are also opposed and the addition $\boldsymbol{k}_{1}+\boldsymbol{k}_{3}$, as $R$ increases, draws $\boldsymbol{k}_{4}$ out of the circle $\left|\boldsymbol{k}_{1}\right|=\left|\boldsymbol{k}_{3}\right|$ and forms a right-tilting hexagon 134. The Fibonacci scenario can follow if in the next $R$ interval, the 7 gets drawn between the 3 and the 4 until the hexagon $(3,4,7)$ is formed. On the other hand, the 5 being drawn between the 1 and the 4 will leave the 1 and the 4 not opposed for a small interval.

And now this is the connection with the dynamical approach. All the algorithms derived from the Hofmeister rule place the nearest phyllo, here 0 , between the two nearest and equidistant ones. In other words, the vectors 0 to $m$ and 0 to $n$, where $m$ and $n$ are parastichies, are equal in length and point to opposite sides of the vertical line through 0. Therefore, their corresponding Van Iterson diagrams will have gaps (no solution exists) in the non-Fibonacci addition $m, n, m+n$ to $n, m+n, 2 m+n$ branch. There will be no gaps in the $m, n, m+n$ to $n, m+n, m+2 n$ branch.

In Figure 36, we display the gap in the $(5,8,13)$-to- $(8,13,21)$ transition. On the Fibonacci branch, namely the one which starts with a $(5,8,13)$ hexagon and ends with a $(8,13,21)$ hexagon, the path is continuous. But, the irregular branch on which the $(5,8,13)$ goes to a $(5,13,18)$, there is no solution over a significant gap. 


\subsubsection{Dynamic models}

A major criticism of the static models of phyllotaxis is that the plant does not spring fully formed from the earth, but instead grows bit-by-bit from the shoot apical meristem (SAM). The SAM is already present at the tip of the plant embryo, which is contained within the seed and has many rapidly dividing cells. Primordia initiate at the edge of the meristem, and as the plant grows, the meristem moves upward and leaves behind previously formed phylla. The botanist Wilhelm Hofmeister published one of the earliest studies of meristems [23] and, from his careful observations, proposed the rules which we have listed in Section 1.1. From these rules, and modifications thereof of Snow and Snow [55, 56] so as to allow for whorls as well as Fibonacci spirals, Douady and Couder carried out a series of numerical simulations reported in [9, 10, 11].

More recently, a much simpler discrete dynamical system with similar outcomes was proposed by Atela, Golé, and Hotton in order to put the Hofmeister rules in a more geometrical framework 2]. As in the static models, the surface of the stem may be approximated by a cylinder. The phase space for the dynamical system is the set of sequences of points $\left\{\boldsymbol{z}_{m}=\left(z_{m}, \theta_{m}\right) \mid m \in \mathbb{N}\right\}$ on the cylinder, where $z_{m}=m \cdot \rho$ for a fixed positive value of the rise $\rho$. The points in any sequence represent the positions of primordia on the cylinder, each primordium residing at a unique level $z_{m}$. The primordium at $\boldsymbol{z}_{m}$ may be thought of as the $m$-th oldest primordium. The dynamical system, defined by its action on the elements of a sequence, takes $\left(z_{m}, \theta_{m}\right) \rightarrow\left(z_{m+1}, \theta_{m}\right)$. That is, each step moves every primordium up one level while having no effect on its angular coordinate. To complete the definition of the map, however, we need to determine the angular coordinate of the point $\boldsymbol{z}_{0}=\left(0, \theta_{0}\right)$. The idea is that $\theta_{0}$ should be chosen so that $\boldsymbol{z}_{0}$ appears in the "least crowded spot." The crowdedness of a spot can be quantified by the distance to the nearest primordium, so let $d\left(\boldsymbol{z}_{m}, \boldsymbol{z}_{n}\right)$ be the Euclidean distance on the cylinder between points $\boldsymbol{z}_{m}$ and $\boldsymbol{z}_{n}$. The distance from a new primordium with angular coordinate $\theta_{0}$ to its nearest neighbor is given by

$$
D\left(\theta_{0}\right)=\min _{m>0} d\left(\boldsymbol{z}_{0}, \boldsymbol{z}_{m}\right) .
$$

Hofmeister's least crowded spot is given by the maximum of $D$ over all angular coordinates $\theta_{0}$. The earlier works by Douady and Couder [9, 10, 11] and Levitov [32, 33, instead assigned to each primordium an inhibitory potential, and the least crowded spot was determined by minimizing the net potential over $\theta_{0}$. These models yield identical results for a potential of the form $d^{-s}$ in the limit $s \rightarrow \infty$.

A main result of $[2$ is that, neglecting arbitrary rotation, simple lattices are asymptotically stable fixed points of this dynamical system. Two criteria are primarily responsible for the selection of fixed point lattices. Due to the fact that $d$ is locally convex, the location of each new primordium is equidistant from two existing primordia. As a result, the fixed-point lattice is rhombic. Additionally, the location of each new primordium has an angular coordinate between the two nearest primordia. One says therefore that the lattice is opposed. It is therefore rhombic, opposed, simple lattices that are stable fixed points of the dynamical system. The rhombic condition implies a fixed point diagram identical to the optimal packing results of Van Iterson, and the opposedness condition breaks the symmetry of the bifurcation points as in the case of Levitov. In other words, the $(n, m+n)$ branch in the Van Iterson diagram of Figure 6 is preferred over the $(m, m+n)$ branch. For a given value of $\rho$, the fixed point could lie on one of many branches of the Van Iterson diagram, but once on a branch it remains there so long as $\rho$ varies adiabatically. So, in most cases, the long-time behavior of this model will result in lattice structures $(m, n)$ that fall along the sequence of successive Fibonacci numbers.

These dynamical algorithms demonstrate that a simple principle based on Hofmeister's rule can generate phyllotactic patterns. Indeed, Hotton, et al. 25] have directly compared the results of the discrete dynamical system of Atela, Gole and Hotton to primordia formation on floral meristms of a sunflower (Helianthus annuus) and shown that there is excellent agreement. Dynamical algorithms have a weakness, however, in that they are not well connected to the chemical or physical mechanisms that are involved in patterning.

\subsection{Biochemical and biophysical mechanisms}

As described in Section 1.3, formation of phyllotactic patterns occurs at the plant tip, called the shoot apical meristem (SAM). Leaves first appear as bulges called primordia on the surface of the SAM (the tunica), in an annular region known as the generative region surrounding the central tip of the SAM. Our goal of 
understanding phyllotactic patterns therefore rests on understanding the mechanisms behind the formation of primordia in the generative region.

As the first application of his theory of the chemical basis for morphogenesis, Alan Turing wrote a beautiful monograph on phyllotaxis [57] in which he proposed reaction-diffusion equations for the formation of the patterns. Turing's work is significant not only in that it was an early proposal of a model for phyllotaxis based on proposed botanical mechanisms, but also in that he introduced in his analysis Fourier wavevector duals to the phyllotactic lattices. In the present work, we make heavy use of this approach. However, morphogens that would correspond to those in Turing's model have not been identified in plants. Although the mechanisms for primordium formation are still not fully understood, the past decade has yielded major experimental advances which point to a rich interaction of biochemical and biomechanical mechanisms.

The cells of primordia grow more quickly than surrounding cells [31. The basic building block for understanding primordium formation is therefore an understanding of the integrated biochemical and physical mechanisms of growth of individual cells that make up the SAM. A plant cell is a pressure vessel [6]. The plant cell wall is a matrix of stiff cellulose microfibrils held together by proteins and polysaccharides. Upon the loosening of this matrix by activators such as expansin, the turgor pressure drives expansion of the cell wall. The relation of growth to turgor pressure for a single cell is given by the Lockhardt model [35: The expansion rate $R$ of a cell wall is proportional to the turgor pressure $P$ when this pressure is above a certain threshold value $Y ; R=m(P-Y)$. Chemicals that change the parameters $m, Y$, or $P$ therefore can give rise to growth. On the other hand, plant cells are tightly bound to each other, so growth may also be influenced by global stresses in the SAM. This sets up a situation in which both biochemical processes that modify cell wall properties, as well as mechanical stresses resulting from turgor pressure and incompatible growth (such as one region growing more quickly than a neighboring region) have the potential to be involved in primordium formation.

The combined turgor pressure of the individual cells that make up the SAM make the SAM itself a pressure vessel. In fact, the group of Kuhlemeier [47] has found that the rheology of the cells in the central region of the SAM (the very apex of the plant shoot tip) differs from that the cells in the surrounding generative region where primordia form. At the standard turgor pressure for plant cells, the composition of the cell walls in the central region make this material a strain-stiffening material, so that although the cells of the central region expand more than those of the cells in the generative region under small strain, under larger strains the cells of the generative region expand significantly more, potentially to the extent necessary for primordium formation.

A variety of chemicals that loosen the cell wall and therefore change the parameters $m$ and $Y$ and allow for growth are activated by the growth hormone auxin [3, 47, 48]. Indeed, studies find that auxin is more concentrated at primordia than in surrounding tissue [20] and show that application of auxin to shoots may induce organ formation [47, 48, 45]. What leads to an inhomogeneous auxin distribution that results in localized primordium formation? Experiments and models by the groups of Kuhlemeier and Meyerowitz implicate auxin transport proteins called PIN1 [28, 48]. In addition to normal diffusion, auxin redistribution may occur through active transport by these proteins. The orientation of PIN1 proteins in a plasma membrane just inside the cell wall determines the direction of transport. The key idea of Kuhlemeier is that a feedback mechanism between auxin concentration and PIN1 orientation results in PIN1 orientation towards cells with a higher concentration of auxin. If this effect is stronger than diffusion, a slight perturbation of a homogeneous auxin concentration is unstable as regions of lower auxin concentration become further depleted of auxin as PIN1 proteins transport the growth hormone to regions of higher concentration.

In the picture thus far, the role of mechanical stresses in primordium formation has been that of a passive response to changes in cell wall composition caused by inhomogeneous auxin concentration. In a myriad of ways in animal cells (e.g. [37]), stresses have been shown or posited to play a more active role in plant meristem growth. Stress-based mechanisms may i) interact with the auxin redistribution mechanism through PIN1 orientation, ii) determine anisotropy in cell growth by affecting the orientation of celluose microfibrils in the cell walls, and iii) directly impact primordium bulge formation through buckling of the tunica.

The feedback mechanism between auxin concentration and PIN1 orientation was not understood in the original proposals of Kuhlemeier and Meyerowitz, whose models simply posited that the cells have some mechanism for detecting the auxin gradient. Working with Arabidopsis, the group of Meyerowitz has, however, shown that mechanical stresses can be a signaling mechanism for PIN1 reorientation [19]. The idea 
is as follows: Suppose that two cells that share a cell wall have differing auxin concentrations. The cell with the higher auxin concentration expands at a larger rate than the cell with the lower auxin concentration. The resulting difference in tension on either side of the cell wall serves as a signal to reorient the PIN1 proteins in the plasma membrane so that they transport auxin to the cell with higher auxin concentration. This mechanism potentially also allows for the global stress state of the SAM resulting from growth and SAM geometry to also impact PIN1 orientation.

Mechanical stresses have also been shown to play a role in determining the degree of anisotropy in cell growth in the SAM. The cellulose microfibrils in the cell wall are laid down by microtubules. As microtubules orient parallel to the main stress direction, cellulose microfibrils end up being oriented in the direction of largest stress at the time of cell wall formation. This allows the cell to resist that stress, but also results in the cells' growing in the direction perpendicular to the main stress. Kwiatkowski and Dumais have shown that cells in the generative region, and particularly around primordia, do exhibit anisotropic growth [31]. Later work of Hamant, et al. showed that microtubules in plant cells align along principal stress directions [18.

Experiments of Paul Green preceding those of Kuhlemeier and Meyerowitz suggested a more direct role for mechanical stresses [16]. A review article of Dumais [12] offers further analysis of the potential role of mechanics in pattern formation in plants. Given the right SAM geometry, the combined effect of the turgor pressure and growth of the individual cells may result in a compressive stress in the generative region, as Green showed occurs in the sunflower floral meristem. Green suggested that primordia result from the buckling of the tunica under this compressive stress. Experiments in which a sunflower floral meristem was placed in a clamp, thus inducing a compressive stress as the meristem grew, did indeed result in bulges forming parallel to the clamp [21].

The experiments of Meyerowitz and Kuhlemeier have been primarily with Arabidopsis and tomato plants, whereas Green also worked with the sunflower. Furthermore, certain phyllotactic patterns not commonly observed on any of these plants are observed in cacti. Although the current dominant thought is that the Kuhlemeier-Meyerowiz instability is responsible for primordium formation, it is possible that different mechanisms dominate in different species. As we discuss in [42, understanding and measuring the time scales involved in the various processes may help in distinguishing between the various mechanisms.

In previous work, we formulated a mathematical model based on the mechanisms proposed by Paul Green [52, 53. Subsquently, by taking a continuum limit of the discrete cell-based auxin-PIN1 model of Jönsson, et al., we formed a pde for this biochemical mechanism and showed that this pde has a similar form to that of the earlier stress-based model [4]. As the auxin-PIN1 mechanism relies on stresses in either a passive or active role, we also formulated a combined model with feedback between the two mechanisms (although the feedback mechanism that we incorporated is different from the more recent experimental suggestion that mechanical stresses are involved in PIN1 reorientation). This combined model allows for the instability to arise from either mechanism alone or a combination of the two mechanisms.

Comparing the results of the analysis of our combined model with phyllotactic patterns, one outcome is particularly relevant to the question of if a combination of interacting mechanisms may be at play: Although studies of phyllotaxis have typically focused on the positions of primordia, Williams [60] points out that plants with the same primordial positions can have patterns dominated by different parastichy spiral numbers due to the shape of the primordia; see Figure 2. Similarly, cacti exhibit various degrees of ribbedness for the same underlying phyllotactic pattern. We have thus aimed to understand what we have called phyllotactic planforms rather than simply the positions of primordia and phylla. A simple model based on only one mechanism produces a simple variety of phyllotactic planforms, and fails to achieve the sequence of planforms with the same underlying phyllotactic lattice as shown in Figure2. In the case in which the natural wavelength of the two instabilities differs slightly, the model predicts a variety of phyllotactic planforms for the same phyllotactic lattice.

\subsection{Mathematical models}

In a series of papers, we analyzed an extension of a pde model of Green, Steele, and Rennich [17] based on Green's proposal for a biophysical origin [52, 53] and later coupled this model to the auxin-PIN1 mechanism [44, 43. In the coupled model, the pde governing the auxin concentration was derived via a continuum approximation of a discrete-cell model for the auxin-PIN1 mechanism developed by Jönsson et al. [28]. 
Here, we review these models and a more recent discrete-cell model of Heisler et al. [19] in which mechanical stresses play a role in determining PIN1 orientation and therefore the auxin field.

In [52, 53, we analyze a pde model for Green's mechanical hypothesis. We model the generative region at a plant apex (Figure 12 as a thin, curved, compressed shell (the plant's outer skin, its tunica) attached to an elastic foundation (the plant's inner corpus of cells) and study how the shell buckles under the influences of compressive stresses that arise from growth. The elastic energy that is minimized by this buckling is a functional $\mathfrak{E}\left(w, \Sigma_{i j}\right)$ of the shell deformation $w$ and an in-plane stress tensor $\Sigma_{i j}$. We employ the Föppl-von Kármán-Donnell (FvKD) equations, which are the Euler-Lagrange equations for the energy $\mathfrak{E}\left(w, \Sigma_{i j}\right)$ and show how a variety of patterns is possible, depending on the anisotropies in the systems and the geometry of the pre-buckling surface. This analysis, however, assumes a uniform pre-buckling stress state and does not take into account the role of auxin suggested by more recent experiments.

In [44, we augment our model to a set of equations for the surface deformation $w$, the Airy potential $F$ (a potential for the stresses $\Sigma_{i j}$ ) and a growth function $g$ related to auxin concentration. The FvKD equations

$$
\begin{aligned}
& \zeta_{m} w_{t}+\Delta^{2} w+P \Delta w+\kappa w+\gamma w^{3}+C \Delta F-[F, w]=0, \\
& \Delta^{2} F+\Delta g-C \Delta w+\frac{1}{2}[w, w]=0,
\end{aligned}
$$

are completed with an equation for the growth function $g$ described below. In (8), $P$ is a uniform stress induced by differential growth, $C$ is the Gaussian curvature of the middle surface of the original, unbuckled tunica shell, $\Delta$ is the Laplacian $\frac{\partial^{2}}{\partial x^{2}}+\frac{\partial^{2}}{\partial y^{2}}$, and the bracket is defined by $[F, w]=F_{x x} w_{y y}+F_{y y} w_{x x}-2 F_{x y} w_{x y}$ (subscripts denote derivatives). The first equation is the force-equilibrium equation of an overdamped shell model as we have neglected a second-order time derivative of $w$, and the second equation is a compatibility equation expressing Gauss's Theorema Egregium. Indeed, in FvKD theory, stresses are linearly related to strains (changes that buckling induces on the metric of the shell's middle surface) so that (8b) relates a potential for changes in the metric $\left(\Delta^{2} F+\Delta g\right)$ to change in Gaussian curvature $\left(-C \Delta w+\frac{1}{2}[w, w]\right)$.

To obtain a pde governing the growth function $g$, we derived a continuum approximation of a discrete-cell model of Jönsson et al. 28] for primordium formation based on the hypothesis of auxin transport by PIN1 proteins. Similar models were developed by Smith et al. [54] and Barbier de Reuille et al. [7]. Here, we review a system of ordinary differential equations proposed by Jönsson et al. [28] and a modification of this model by Heisler et al. [19] which included stress-induced PIN1 orientation. In the model of [28], a planar generative region (representing Region 2 of Figure 12 in the tunica is taken to be made up of a network of cells, which we picture as a simplified hexagonal lattice in Figure 17. The auxin concentration $a_{i}$ in cell $i$ is governed by the following system of ordinary differential equations:

$$
\frac{d a_{i}}{d t}=c_{a}-d_{a} a_{i}+\sum_{k \in N_{i}} D\left(a_{k}-a_{i}\right)+\sum_{k \in N_{i}}\left(P_{k i} h\left(a_{k}\right)-P_{i k} h\left(a_{i}\right)\right)
$$

where the summations are taken over the set $N_{i}$ of cells that share a wall with cell $i$. In the system (9), the term $c_{a}$ represents the constant production of auxin, and the term $-d_{a} a_{i}$ represents auxin degradation. The term $\sum_{k \in N_{i}} D\left(a_{k}-a_{i}\right)$ represents normal diffusion with diffusion coefficient $D$. The final term, $\sum_{k \in N_{i}}\left(P_{k i} h\left(a_{k}\right)-P_{i k} h\left(a_{i}\right)\right)$, is the so-called PIN1 up-regularization term, since the protein PIN1 in the plasma membrane can polarize so that auxin may be transported from cells with lower to cells with higher auxin concentrations, a reverse diffusion. For any $(i, k), P_{i k}$ is the PIN1 concentration near the cell wall of cell $i$ that is adjacent to cell $k$. The terms $P_{k i} h\left(a_{k}\right)$ give the rate of auxin flux into the cell due to up-regularization, and the terms $P_{i k} h\left(a_{i}\right)$ give the corresponding rate of auxin flow out of the cell $i$. In [28], $h\left(a_{i}\right)$ is taken to be $h\left(a_{i}\right)=a_{i}$, whereas in [19], $h\left(a_{i}\right)$ is taken to have the Michaelis-Menten form $h\left(a_{i}\right)=a_{i} /\left(\kappa+a_{i}\right)$.

In order to close the system (9p), a relation between the concentrations $P_{i j}$ of PIN1 on the plasma membranes and the auxin concentrations $a_{i}$ is required. In a process called PIN1 cycling, PIN1 protein molecules cycle between being bound to the plasma membrane or being in the interior of the cell. Consider two neighboring cells, cell $i$ and cell $j$, with a concentration of PIN1 interior to the cell and PIN1 on each plasma membrane. Denoting the interior PIN1 concentration by $P_{i}$ and the PIN1 concentration in cell $i$ 
adjacent to cell $j$ by $P_{i j}$, the time responses of $P_{i j}$ and $P_{i}$ are taken to be

$$
\begin{aligned}
\frac{d P_{i j}}{d t} & =f\left(a_{i}, a_{j}\right) P_{i}-k_{2} P_{i j}, \\
\frac{d P_{i}}{d t} & =\sum_{k \in N_{i}}\left(k_{2} P_{i k}-f\left(a_{i}, a_{k}\right) P_{i}\right) .
\end{aligned}
$$

The key property of the function $f\left(a_{i}, a_{j}\right)$ is that it be an increasing function of $a_{j}$, so that a higher concentration of auxin in the neighboring cell $j$ will cause an increase in the growth rate of $P_{i j}$ through the term $f\left(a_{i}, a_{j}\right) P_{i}$. Simultaneously, an internalization process $-k_{2} P_{i j}$ takes PIN1 back to the cell interior with rate constant $k_{2}$. Note that (10a) and (10b) together imply that the total PIN1 concentration in cell $i$, $P=P_{i}+\sum_{k \in N_{i}} P_{i k}$ is constant, which we assume to be the same in all cells.

The models of Jönsson et al. [28] and Heisler et al. [19] differ in the choice of the function $f\left(a_{i}, a_{j}\right)$. In [28, the authors assume a linear model, namely

$$
f\left(a_{i}, a_{j}\right)=f\left(a_{j}\right)=k_{1} a_{j} .
$$

Later evidence of the effect of mechanical stress on PIN1 cycling [18, 19] led Hamant, et al. in [19] to write $\left.f\left(a_{i}, a_{j}\right)=f\left(s_{i j}\left(a_{i}, a_{j}\right)\right)\right)$ as a function of the stress $s_{i j}$ in the wall of cell $i$ adjacent to cell $j$. This stress, in turn, is a function $s_{i j}\left(a_{i}, a_{j}\right)$ of the auxin concentrations in the cells $i$ and $j$ since the auxin concentrations affect the stiffness of the cell walls. In particular, $s_{i j}$ is taken to have the Hookean form

$$
s_{i j}\left(a_{i}, a_{j}\right)=E\left(a_{i}\right) \epsilon_{i j}\left(a_{i}, a_{j}\right),
$$

where the elastic coefficient is a decreasing function of $a_{i}$;

$$
E\left(a_{i}\right)=E_{\min }+\frac{\left(E_{\max }-E_{\min }\right) k_{3}^{m}}{a_{i}^{m}+k_{3}^{m}},
$$

where $m$ is a small integer. The strain $\epsilon_{i j}$ depends on the force $T / A_{0}$ exerted by internal turgor pressure $T$ and the stiffnesses of the two sides of the cell walls;

$$
\epsilon_{i j}=\frac{T / A_{0}}{E\left(a_{i}\right)+E\left(a_{j}\right)} .
$$

Relations (11) and (13) yield

$$
s_{i j}=\frac{T / A_{0}}{1+\frac{E\left(a_{j}\right)}{E\left(a_{i}\right)}} .
$$

Finally, Heisler et al. [19] take $\left.f\left(s_{i j}\left(a_{i}, a_{j}\right)\right)\right)$ to be an integer power of $s_{i j}$;

$$
\left.f\left(s_{i j}\left(a_{i}, a_{j}\right)\right)\right)=s_{i j}^{n} .
$$

In simulations, $n$ is taken to be 2. Since $E\left(a_{j}\right)$ is a decreasing function of $a_{j}, f\left(s_{i j}\left(a_{i}, a_{j}\right)\right)$ is an increasing function of the auxin concentration $a_{j}$ in the cell $j$ neighboring cell $i$. According to (10a), an increase in $a_{j}$ leads to an increase in the rate at which PIN1 incorporates into the wall of cell $i$ adjacent to cell $j$, and consequently, according to the system (9), an increase in the rate at which auxin is transported by these proteins from cell $i$ to cell $j$. The net result is that an increase in $a_{j}$ results in an increased transport of auxin from cell $i$ to cell $j$, acting like reverse diffusion.

Equations $(9)$ and 10 form a closed system for the variables $a_{i}, P_{i}$ and $P_{i j}$. A simplification may be made by assuming, consistent with data given in Table S2 of Jönnson, et al. [28, that the PIN1 relaxation time $k_{2}^{-1}$ is much smaller than the auxin concentration relaxation time $d^{-1}$ and that the PIN1 concentration $P_{i j}$ responds adiabatically to changes in $a_{i}$. Equation 10 then implies that

$$
P_{i j}=\frac{f\left(a_{i}, a_{j}\right)}{k_{2}} P_{i}
$$


which together with the observation that $P=P_{i}+\sum_{k \in N_{i}} P_{i k}$ is constant implies that

$$
P_{i j}=P \frac{f\left(a_{i}, a_{j}\right)}{k_{2}+\sum_{k \in N_{i}} f\left(a_{i}, a_{k}\right)}
$$

a expression for the PIN1 concentrations $P_{i j}$ in terms of the auxin concentrations. Substituting this expression back into $(9)$ gives a closed system of equations for the concentrations $a_{i}$ :

$$
\frac{d a_{i}}{d t}=c_{a}-d_{a} a_{i}+D \sum_{k \in N_{i}}\left(a_{k}-a_{i}\right)+P \sum_{k \in N_{i}}\left(h\left(a_{k}\right) \frac{f\left(a_{k}, a_{i}\right)}{k_{2}+\sum_{j \in N_{i}} f\left(a_{k}, a_{j}\right)}-h\left(a_{i}\right) \frac{f\left(a_{i}, a_{k}\right)}{k_{2}+\sum_{j \in N_{i}} f\left(a_{i}, a_{j}\right)}\right)
$$

In [44, we take advantage of the fact that the auxin concentration fluctuations found in 28, occur over many cell diameters. This allows us to recast the discrete system (16) of 28] in a continuum pde approximation by replacing the set of concentrations $a_{i}$ by a continuous field $a(x, y, t)$. In [44], we derive the continuum approximation using a square lattice of cells, but the hexagonal lattice that we use here results in more rotational symmetry in the resulting system of pde's. If $\left(i_{1}, i_{2}\right)$ are the coordinates of the center of cell $i$, so that $a_{i}=a\left(i_{1}, i_{2}, t\right)$, we write $a\left(x=i_{1} h_{0}, y=i_{2} h_{0}, t\right)=a\left(i_{1}, i_{2}, t\right)$, where $h_{0}$ is the cell diameter (that is, the distance from the center of a hexagonal cell to the nearest side). For a hexagonal lattice, the difference term $D \sum_{k \in N_{i}}\left(a_{k}-a_{i}\right)$, for example, becomes

$$
\begin{aligned}
& D\left(a\left(i_{1}+h_{0} \frac{1}{2}, i_{2}+h_{0} \frac{\sqrt{3}}{2}\right)+a\left(i_{1}-h_{0} \frac{1}{2}, i_{2}+h_{0} \frac{\sqrt{3}}{2}\right)+a\left(i_{1}+h_{0} \frac{1}{2}, i_{2}-h_{0} \frac{\sqrt{3}}{2}\right)\right. \\
& \quad+a\left(i_{1}-h_{0} \frac{1}{2}, i_{2}-h_{0} \frac{\sqrt{3}}{2}\right)+a\left(i_{1}+h_{0}, i_{2}\right)+a\left(i_{1}-h_{0}, i_{2}\right)-6 a\left(i_{1}, i_{2}\right)
\end{aligned}
$$

Expressed as a Taylor series up to fourth order, this is

$$
3 \frac{h_{0}^{2}}{2 !} \nabla^{2} a+9 \frac{h_{0}^{4}}{4 !} \nabla^{4} a .
$$

The hexagonal lattice allows us preserve rotational symmetry up to $O\left(h_{0}^{4}\right)$. A square lattice would only preserve this invariance to $O\left(h_{0}^{2}\right)$. To expand the terms related to PIN1 in (16), it is convenient to introduce the fractional PIN1 concentration field $G(x, y, t)$, where

$$
G(x, y, t)=G\left(i_{1} h_{0}, i_{2} h_{0} ; j_{1} h_{0}, j_{2} h_{0} ; t\right)=\frac{f\left(a_{i}, a_{j}\right)}{k_{2}+\sum_{k \in N_{i}} f\left(a_{i}, a_{k}\right)} .
$$

Summing up over the six cells adjacent to the $i$ th cell, labelled $\left(i_{1}, i_{2}\right)$ and expanding to forth order gives

$$
P h_{0}^{2} \nabla\left(a^{2} \nabla\left(\frac{G}{a}\right)\right)+P \frac{h_{0}^{4}}{12}\left(a \nabla^{4} G-G \nabla^{4} a\right) .
$$

The fact that the first term in $(19)$ is gradient so that the balance between the time derivative of $a(x, y, t)$ and this quadratic term is conservative is a reflection of the fact that, in the absence of the production and degradation terms in (7), the discrete model conserves $\sum a_{i}$. To $O\left(h_{0}^{2}\right)$, the first term in $(9)$ becomes a Laplacian because it involves nearest neighbor interactions; the second term involves next to nearest neighbor interactions and thus leads to a single gradient.

Next, we expand about the spatially uniform constant state $a_{0}=\frac{c_{a}}{d_{a}}$ by writing

$$
a(x, y, t)=a_{0}\left(1+f_{0} g(x, y, t)\right),
$$

where $f_{0}$ is a coefficient of expansion, and the field $g$ is a measure of auxin fluctuation. We emphasize that, in this analysis, we are dealing with fluctuations in the auxin concentration which are small with respect to the overall auxin concentration level. While the local auxin concentration field $a(x, y, t)$ is never negative, negative values of $g(x, y, t)$ are possible and just mean that there is an auxin depletion at that point.

Substituting (18) to 20 into (16) and expanding in powers of $f_{0} g$ gives

$$
\zeta_{g} g_{t}+D_{g} \Delta^{2} g+H \Delta g+d g+\delta g^{3}+\kappa_{1} \nabla(g \nabla g)+\kappa_{2} \nabla\left(\nabla g \nabla^{2} g\right)=0,
$$


where $H \sim h_{0}^{2}\left(T-D_{g}\right)$ measures the strength of auxin transport $(T)$ versus diffusion $\left(D_{g}\right)$. Equation 21 . has a form that describes pattern formation in many systems. In fact, it closely mimics the stress-equilibrium equation 8a), the differences lying in the nonlinear terms and the interpretation of the parameters in the linear terms.

In (21), we have neglected cubic terms that include gradients of $g$ as modeling them with $g^{3}$ makes no qualitative difference. We will see in Section 3 that retaining gradient terms in the quadratic coefficients is important, as otherwise it is difficult to achieve "pushed" fronts. (21):

The final set of model equations that we derive in [44] consists of the FvKD system (8) together with

$$
\begin{aligned}
& \zeta_{m} w_{t}+\Delta^{2} w+P \Delta w+\kappa w+\gamma w^{3}+C \Delta F-[F, w]=0, \\
& \Delta^{2} F+\Delta g-C \Delta w+\frac{1}{2}[w, w]=0, \\
& \zeta_{g} g_{t}+D_{g} \Delta^{2} g+H \Delta g+d g+\delta g^{3}+\kappa_{1} \nabla(g \nabla g)+\kappa_{2} \nabla\left(\nabla g \nabla^{2} g\right)-b \Delta F=0,
\end{aligned}
$$

where equation (22c) contains an added term $-b \Delta F$. It says that an increase in the trace of the stress matrix $\Delta F$, a measure of how cells may be pulled apart, provides an additional contribution to the growth rate $g$.

Either (22a) and (22b) or (22c) may produce an instability of the homogeneous state to a pattern. If both the elastic instabilities of (22a) and (22b) and the auxin instability of (22c) are active, the possibly different natural wavelengths of the patterns that would result from either instability alone allow for differences in phyllotactic configurations (the underlying lattice) and the surface deformation. The result is phyllotactic planforms in which the phylla are diamonds rather than squares. In 44] we analyze a variety of scenarios in which the elastic and auxin instabilities may cooperate or compete.

For the remainder of this review, however, we concentrate of the case for which the auxin instability and therefore the auxin field dominates. This arises when the differential growth induced compressive stresses at the generating annulus are strongly subcritical so that, for all intents and purposes, the Airy potential $F$ and the corresponding surface normal deformation field $w$ of the tunica are slaved to the auxin fluctuation induced strain field $g$. If, in addition, we assume that $\kappa_{2} k_{0}^{2}$ is small compared to $\kappa_{1}$, where $k_{0}$ is the preferred wavenumber of the auxin instability, we can adiabatically eliminate the $w, F$ fields and deal solely with the equation for the $g$ field. A little rescaling puts this into the canonical form

$$
u_{t}=-\left(\nabla^{2}+1\right)^{2} u+\mu u-\beta \nabla(u \nabla u)-u^{3} .
$$

Because previous work 44 indicates that, except for the case when the two instabilities coexist, there is not much qualitative difference in the outcomes, it is 23 which we analyze in depth in Section 3 . The reason for this choice is that it is essential that we conduct careful simulations over large regions and long times in order to verify that the patterns suggested by asymptotic methods described in Section 3.3 are in fact realized. The conservation nature of the PIN1 term in (7) means that the linear reverse diffusion and quadratic terms enter as divergences. The results we show in the next section are relatively robust over an open set of the coefficients $\mu, \beta$, or indeed over changes in the equation itself as long as the quadratic terms enter with derivatives. If one were to include a quadratic term (and nowhere in the model derivation is there any reason to do so), say proportional to $u^{2}$, then 23. would have additional spatially constant solutions which would compete with hexagonal configurations.

\section{Analysis and Simulations}

The goal of this section is to gain as much understanding as possible from the model we have formulated in Section 2.4. To this end, we will use the reduction (23). The geometrical picture we have in mind is motivated by the seed formation in a sunflower (see Figure 9a). The pattern is initiated at some outer boundary by the floret configuration that has recently been made there. It propagates inward. In the language of Section 1.3 , the outer boundary of the region of already developing florets is Region 3 . The inner region of slowly growing cells is Region 1. At the boundary, the generative annulus, Region 2, consisting of faster growing cells, forms with uniform auxin concentration and, as explained in Section 2.3, the rheology of the cells in this generative annulus differs from that of the cells in Region 1. The uniform auxin concentration 
in this newly formed annulus corresponds to the solution $u=0$ of 23 and is unstable. A new pattern will form in this annulus, and its structure will be influenced by the pattern that already exists on its outer boundary. The process repeats. New auxin field maxima are thus created created annulus-by-annulus along a circular front until all of Region 1 has been converted first to Region 2 and then to the patterned Region 3. It is relatively easy to modify the model to handle other similar scenarios such as an outward propagating pattern with or without underlying plant growth which would be captured by the addition of an advection term in (23).

We use two investigative tools, direct numerical simulation and analysis based on the ideas of center manifold theory. Each has its strengths and weaknesses, but understanding gained from one informs the other.

In Section 3.1, we outline the method that we use for simulating (23), define the information that we extract from the completed solution $u(r, \theta)$, and indicate how the method can be easily adapted for use not only on disks, but for any surface of revolution. For reasons that we explain both in Sections 3.1 and 3.3, it turns out to be useful to introduce a companion equation to 23 which, near onset, namely for small $\mu$, has virtually the same dynamics, but is a gradient flow. In Section 3.2, we present the main outcomes of our simulations. In the order that they are presented, the key results are the following: (i) Equation $\sqrt{23}$ and its companion gradient flow lead to sunflower patterns which are not only visually identical but which, under Fourier analysis, have the same properties. (ii) At each radius $r$, the field $u(r, \theta)$ is dominated by Fourier amplitudes $A_{m}(r)$ corresponding to either three modes with wavevectors $\boldsymbol{k}_{m}=\left(l_{m}, m / r\right), \boldsymbol{k}_{n}=\left(l_{n}, n / r\right)$, $\boldsymbol{k}_{m+n}=\boldsymbol{k}_{m}+\boldsymbol{k}_{n}$, or four modes $\boldsymbol{k}_{n}-\boldsymbol{k}_{m}, \boldsymbol{k}_{m}, \boldsymbol{k}_{n}, \boldsymbol{k}_{m+n}$ consisting of two overlapping triads. (iii) The set $\{m\}$ of dominant modes is a subset of the integers defined by the Fibonacci rule. (iv) The radial interval $8<r<233$ is split up into intervals $R_{j}, R_{j+1}=R_{j} \phi$, where $\phi$ is the golden number. Let $R_{m}, R_{n}$ be such successive radii. At $R_{m}$ and $R_{n}$, the pattern is hexagonal, dominated by three modes, $\boldsymbol{k}_{n-m}, \boldsymbol{k}_{m}, \boldsymbol{k}_{n}$ at $R_{m}$ and $\boldsymbol{k}_{m}, \boldsymbol{k}_{n}, \boldsymbol{k}_{m+n}$ at $R_{n}$, with equal amplitudes, and wavenumbers equal to a preferred wavenumber $k_{0}$. Between $R_{m}$ and $R_{n}$, the modes with wavevectors $\boldsymbol{k}_{n-m}$ and $\boldsymbol{k}_{m+n}$ have smaller amplitudes and wavenumbers far from $k_{0}$. (v) The total pattern is self-similar. The amplitudes and wavevectors of both the active Fibonacci modes (and, it turns out, of all other passive integer modes) in any one interval can be mapped into those in the neighboring interval by $A_{n}(r \phi)=A_{m}(r), l_{n}(r \phi)=-l_{m}(r)$, where $m$ and $n$ are successive Fibonacci numbers. As $m$ and $n$ increase, $n / m$ approaches $\phi$. In the regions in which they are dominant, all of the wavevectors paths $\left\{l_{m}(r), m / r\right\}_{m}$ lie on top of each other. (vi) The reasons that the dominant modes in each interval are selected via the Fibonacci rule become clear when one sees how the wavevectors $\boldsymbol{k}_{n-m}=\boldsymbol{k}_{n}-\boldsymbol{k}_{m}, \boldsymbol{k}_{m}, \boldsymbol{k}_{n}, \boldsymbol{k}_{m+n}=\boldsymbol{k}_{m}+\boldsymbol{k}_{n}$ generated by Fibonacci addition and the wavevectors of all other nonlinearly generated modes such as $\boldsymbol{k}_{2 n-m}=\boldsymbol{k}_{n-m}+\boldsymbol{k}_{n}$ move as functions of $r$. The wavenumbers of the former all approach, for some value of $r$, the preferred wavenumber $k_{0}$. The latter never do.

Because our understanding of the patterns is greatly facilitated by a Fourier analysis, it is natural to introduce, as we do in Section 3.3, a center manifold approach familiar from traditional means of pattern analysis. Indeed, in many of our earlier works, this was the primary investigative tool [52, 53]. The idea is to replace the pde 23) (or its gradient flow companion) with a set of amplitude equations for the amplitudes $A_{m}$ of the Fourier modes. Near the onset of instability, one can identify a set of shapes and configurations to which the uniform solution, here $u=0$, is most unstable. In systems of large aspect ratio (systems for which the domain size is much larger than the length scales involved in the most unstable modes) with rotational and translational invariance, the unstable shapes are Fourier modes $e^{i \boldsymbol{k} \cdot \boldsymbol{x}}$, where the the wavenumber $k=|\boldsymbol{k}|$ is close to some preferred value $k_{L}$ (for $(23), k_{L}=1$ ) determined by linear stability analysis. For $\mu>0$, we would designate all modes whose wavevectors $\boldsymbol{k}$ have wavenumbers equal to or close to $k_{L}$ as part of an active set $A$ and designate all others as part of the passive set. We indicate in Section 3.3 what the resulting equations for the amplitudes of the active modes would be if the geometry is planar and the orientation of the planform is determined by some external bias (such as boundary conditions). The quadratic terms in (23) and its companion gradient flow preferentially choose hexagon shapes because the quadratic interaction of two modes $e^{i \boldsymbol{k}_{j} \cdot \boldsymbol{x}},\left|\boldsymbol{k}_{j}\right|=k_{L}, j=1,2$ can give rise to a third mode $e^{i \boldsymbol{k}_{3} \cdot \boldsymbol{x}}$ with $\boldsymbol{k}_{1}+\boldsymbol{k}_{2}+\boldsymbol{k}_{3}=0$ and $\left|\boldsymbol{k}_{3}\right|=k_{L}$ if $\boldsymbol{k}_{1}$ and $\boldsymbol{k}_{2}$ are $120^{\circ}$ apart. In such cases, it turns out that the coupling coefficient $\tau\left(\boldsymbol{k}_{1}, \boldsymbol{k}_{2}, \boldsymbol{k}_{3}\right)$ measuring the strength of the quadratic interaction is symmetric in all indices so that the resulting amplitude equations for the amplitudes $A_{1}, A_{2}, A_{3}$ are gradient even if the original pde is not gradient. A prime example of this is convection patterns. The governing Oberbeck-Boussinesq equations are not gradient, but, near onset, for large Prandtl numbers, the amplitude equations are. In other words, in planar geometries with hexagonal 
patterns, the amplitude equations for 23) and its gradient flow companion are both the same and are gradient. Consequently, there is a natural free energy, $E\left(A_{1}, A_{2}, A_{3}\right)$, and the winning configurations are those that minimize $E$.

But, phyllotactic patterns in disk geometries which are laid down annulus-by-annulus do not quite conform to that prescription. The main reason for this is that, as the pattern advances from annulus of one radius to another, the active modes change. So, the object that we define in Section 3.1 as the center manifold also evolves. Moreover, whereas at all the radii $R_{j}$ at which hexagonal patterns dominate, the resulting amplitude equations are gradient, in between these radii they are not. We have therefore to try to understand why it is that the whole pattern does indeed behave as if it is nearly gradient. This we do both in Section 3.3, where we derive the appropriate set of amplitude equations, and in Section 3.4, where we show that the results obtained from an amplitude equation analysis are completely consistent with those that we obtain from simulations.

But, while the amplitude equations can give the right answers, the simulations are absolutely necessary to delineate the circumstance for which the continuum of solutions parameterized by the generative radius $R$ is actually realized. Bias (Koiter coined the phrase geometric imperfection, in mathematics it became known as imperfect bifurcation) plays an important role. The new pattern at $R$ is heavily influenced by the existing pattern at the neighboring annulus $R+d R$. In gradient systems, the energy minimum of the latter lies in the basin of attraction of the energy minimum of the former so it is natural for the solution to follow the continuum. But the energy landscape also has other minima (see Result 6, Section 3.2 and Result 4, Section 3.4) which may be accessed if perturbations are large enough. Moreover, as we show in Section 3.3, there are other modes which may not have been included in an amplitude equation analysis which can become linearly unstable. If their growth times are sufficiently fast, they too can interrupt the continuum of amplitude equations solutions. Thus, we need the simulations to be sure that the amplitude equations do indeed describe the evolving patterns.

We also raise the more general question, using Fibonacci patterns as the example, of the need to adapt center manifold analyses to cover those situations for which the center manifold itself changes slowly. In this regard, we present a novel and exciting conjecture as to how to view the center manifold.

\subsection{Simulations: What we do, what we measure}

The choice of geometry and boundary conditions for our simulation of 23 is motivated by the seed formation stage of the sunflower (Figure 9 $\mathrm{a}$ ). As we have recounted, sunflowers are formed in two stages. In the first stage, flowers are initiated at the generative annulus, which increases in radius. As a result, the older flowers with the lowest parastichy numbers are on the outside. The newly formed higher parastichy numbers are on the inside. At a certain point, however, the center region consisting of mushy undifferentiated cells undergoes a phase transition and, from the outside in, the seeds or florets are laid down by a front which moves inward. The parastichy numbers decrease with decreasing radius. Because this process occurs rapidly, on a time scale of days, the pattern which is laid down at a certain radius remains at that radius. The diameter of the plant at this stage is on the order of millimeters. Subsequent growth to the state we picture as a fully developed sunflower with a size of 10-15 centimeters takes place over many weeks. Thus at the seed-making stage, the optimal packing property which the pattern manifests when first laid down remains visible.

To simulate this situation, we initiate a hexagonal pattern at a radius of approximately 233 units with dominant parastichy numbers $(89,144)$ and allow it to invade the interior of the disk, on which the initial state is $u=0$. To show that it is the Fibonacci rule rather than the actual starting numbers which influence the parastichy number progression, we run concurrently a computation with starting parastichy numbers $(76,123)$ beginning at a radius of 199 units. Such an arrangement would arise if the starting parastichies at the flower-making stage were $(1,3)$ rather than $(1,2)$. The starting pattern is determined from a hexagonal $(89,144,233)$ shape as calculated from the amplitude equations (which we discuss in Section 3.3). The front is allowed to evolve by the pde (23) on a cylinder with the outer radius until it reaches a steady state. The amplitude equation analysis is so accurate that the hexagonal pattern hardly changes at all. The idea behind the choice of initial conditions is that the seed pattern is most heavily influenced by the flower pattern most recently laid down before the seed-making phase begins.

We then propagate the solution inwards until it reaches a radius of approximately 13 units. The evolving front chooses its own speed. It also appears to choose a wavenumber $k_{0}$ which is close to the wavenumber 
$k=1$ chosen by a linear stability analysis of the $u=0$ solution in 23 . As the pattern behind the front evolves, we observe that it has the following character. At a countable set of radii $\{233,144,89, \ldots\}$, it is hexagonal. Between these radii, it is basically rhombic. For an open set of parameters in the $(\mu, \beta)$-plane, the front is also pushed. We will have more to say about this later.

Once the pattern has reached the inner radius, the total pattern in the disk reaches a steady state. We analyze it by writing

$$
u(r, \theta)=\sum_{m} a_{m}(r) \exp \left(i\left(\phi_{m}(r)+m \theta\right)\right)+(*)
$$

over non-negative integers $\{m\}$ where $a_{m}(r)$ and $\phi_{m}(r)$ are real. The sequence $\left\{a_{m}\right\}$ is the sequence of amplitudes. The radial derivatives of the real phase $\ell_{m}=\phi_{m}^{\prime}(r)$ give us the local radial wavenumbers. From the wavenumber pairs $\left(\ell_{m}, m\right),\left(\ell_{n}, n\right)$ with the largest amplitudes at any radius $r$, namely the parastichies, we can determine the local rise and divergence angle from the formulae

$$
\begin{gathered}
2 \pi d=2 \pi \frac{\ell_{m} p-\ell_{n} q}{\ell_{m} n-\ell_{n} m} \\
\rho=\frac{1}{r} \frac{q n-p m}{\ell_{m} n-\ell_{n} m}
\end{gathered}
$$

(see 39 for derivation). Because $m, n$ are coprime, we can always find a $p$ and a $q$ such that $q n-p m= \pm 1$. We make the unique choice of $p$ and $q$ which guarantees the angle $2 \pi d$ is between 0 and $2 \pi$. At radii where the pattern is hexagonal, there will be three dominant modes with wave vectors $\boldsymbol{k}_{m}, \boldsymbol{k}_{n}$, and $\boldsymbol{k}_{m+n}=\boldsymbol{k}_{m}+\boldsymbol{k}_{n}$. Any pair of the three wave vectors may be used to calculate $d$ and $\rho$.

Solving (23) numerically is challenging, especially since our computation will cover an area containing on the order of $10^{4}$ maxima of the field $u$. The plant's choice of geometry makes it difficult to use a spectral or pseudo-spectral method, and so we must fall back on finite difference methods to approximate the spatial derivatives. On the other hand, this leads to significant stiffness in the difference operators since the pde is fourth-order in space. Furthermore, the spatial derivatives in the nonlinear terms make it difficult to obtain stability even with modest time-steps. In order to address these difficulties, we try to reorganize (23) into a form which is as close to a gradient flow as possible. We rewrite 23 as

$$
\frac{\partial u}{\partial t}=\left(\mu-\left(\nabla^{2}+1\right)^{2}\right) u-\frac{\beta}{3}\left(1+\frac{\gamma}{2}\right)\left(2 u \nabla^{2} u+|\nabla u|^{2}\right)-\frac{\beta \gamma}{2}|\nabla u|^{2}-u^{3} .
$$

Taking $\gamma=1$, we obtain the original equation. Taking $\gamma=0$, we obtain the gradient flow

$$
\begin{gathered}
\frac{\partial u}{\partial t}=\left(\mu-\left(\nabla^{2}+1\right)^{2}\right) u-\frac{\beta}{3}\left(2 u \nabla^{2} u+|\nabla u|^{2}\right)-u^{3}=-\frac{\delta E}{\delta u} . \\
E=\int\left(-\frac{1}{2} \mu u^{2}+\frac{1}{2}\left(\left(\nabla^{2}+1\right) u\right)^{2}-\frac{\beta}{3} u|\nabla u|^{2}+\frac{1}{4} u^{4}\right) .
\end{gathered}
$$

Our simulations of 23 will take advantage of this decomposition by using the gradient part of (27) with $\gamma=1$ to obtain our first approximation to the solution $u$ and then adopting an iterative procedure to calculate the correction due to the remainder $-\beta|\nabla u|^{2}$. It turns out that there is very little difference in the solutions of (27) and (23), a result we will illustrate by the first graphs in the following Section 3.2 For reasons that we explain later, for small values of $\mu$, namely near the onset of instability of the unstable solution $u=0,23$ behaves very much as if it were a gradient flow. We now describe how we solve (27).

First, in a manner similar to [14, we construct a discrete approximation to the energy (29) using quadrature for the integration and finite differences for the derivatives. By taking a difference of sums and manipulating the resulting expression using summation by parts, we can transform this approximation into a form reminiscent of a variational derivative. In this way, we determine a discrete variational derivative of the energy, which we can use as an approximation for the gradient term in our numerical method. The benefit of this approach is that the numerical method inherits any continuous variational properties of the pde, in particular, that the solution is bounded a priori by norms of the initial condition. Thus we can explicitly avoid numerical instability. More details are contained in [46]. Having taken a time step of the gradient 
part of 27) with $\gamma=1$, we can implicitly compute the correction to the step using the term $-(\beta / 2)|\nabla u|^{2}$ if necessary.

It is also possible to extend the whole calculation to arbitrary axisymmetric surfaces, which we exploit in later sections. This captures the fact that the radius of the generative annulus may change arbitrarily during the growth of the plant, and the radius in part determines the pattern being laid down. Any axisymmetric surface can be described as a surface of revolution, which can in turn be describe by its cross-section. Let $z$ be the arc length axially along the cross-section and $r(z)$ be the distance to the axis of the surface. Also, let $\theta$ be the angle about the axis. This is a convenient choice of coordinates since the line element becomes $d s^{2}=d z^{2}+r^{2}(z) d \theta^{2}$, which gives the gradient

$$
\nabla=\left(\frac{\partial}{\partial z}, \frac{1}{r(z)} \frac{\partial}{\partial \theta}\right)
$$

and the Laplacian

$$
\nabla^{2}=\frac{\partial^{2}}{\partial z^{2}}+\frac{r^{\prime}(z)}{r(z)} \frac{\partial}{\partial z}+\frac{1}{r^{2}(z)} \frac{\partial^{2}}{\partial \theta^{2}} .
$$

In the case $r(z)= \pm z$, these give the corresponding operators on the disk in polar coordinates. For instance, we use $r(z)=233-z$ to compute on the disk illustrated in Figure 18 . In the case $r(z)=r_{0}$, for some constant $r_{0}>0$, these give the corresponding operators on the cylinder of radius $r_{0}$. In the case $r^{\prime}(z)=c$, for $0<c<1$, the surface forms part of a cone. We use such a surface in Section 3.5 to simulate a slowly varying radius of the generative annulus. Fortunately, it is straightforward to modify our numerical methods to compute solutions of $(23)$ for arbitrary $r(z)$.

In addition, for solutions to both (23) and (27), we measure $\epsilon(r), \eta(r)$ and $\nu(r)$, the local energy, local packing density and front speed respectively, which all change with $r$. The front speed $\nu$ is the instantaneous front speed at a particular radius. To find this speed, we locate the front by computing the mean of $u^{2}$ around the circumference. This gives a function of $r$ which has a near-constant positive value in the patterned region and is zero in the unpatterned region. By finding the location of the half-maximum value, it is possible to locate the front. The velocity is then calculated using a finite difference applied to the position. The local energy $\epsilon$ is the energy density of the local lattice, computed using a discrete approximation to (29). The local packing efficiency $\eta$ is the largest fraction of the local cylinder, representing the pattern in a narrow annulus at radius $r$, that can be covered by identical circles centered at maxima of the local lattice. This quantity is proportional to $1 /\left|\boldsymbol{k}_{m} \times \boldsymbol{k}_{n}\right|$, where $m, n$ are the parastichy numbers at that radius. A complete derivation can be found in 39 .

Finally, in order to determine what kind of fixed point the apparently stationary pattern of the pde (27) is, we continue the calculation for up to five times the time it takes the front to go from the outer to the inner radius. Because the pattern is so large at this stage compared with the local wavelength, the geometry will look locally planar, and for planes the energy minimizing planform is hexagonal. On a very long time scale, defects begin to appear which we see by temporary but dramatic sudden decreases in the free energy. These defects are part of an effort to reorganize the pattern into hexagonal patches each with their local orientations. Thus we conclude that Fibonacci patterns are not global attractors but are actually saddle points with strongly attracting stable manifolds and weakly repelling unstable ones. They are long-lived and therefore potentially observable in many contexts.

\subsection{The results of the simulations: Fibonacci patterns}

We now present our findings from direct numerical simulation of our model (27) in the cases $\gamma=0$ and $\gamma=1$, which we will call gradient and nongradient, respectively.

Result 1. Figures 18 to 20. A remarkable outcome, which is not at all clear a priori, is that the pattern laid down by the front is nearly identical for the gradient and the nongradient cases. In Figure 18, we present the results of two simulations on the disc $r<233$ initiated at the boundary $r=233$ with a pattern having parastichy numbers $(89,144)$. The two simulations are nearly indistinguishable qualitatively. The similarity, however, does not stop there.

In Figure 19, we present amplitude data gathered from both these simulations. In addition to being again visually indistinguishable, quantitatively we observe the following. By scaling the radius as $r / \varphi^{i}$, where $i$ is the index of the circumferential wavenumber $m_{i}$ in the regular Fibonacci sequence and $\varphi=(1+\sqrt{5}) / 2$ is 
the golden ratio, we find that both the gradient and nongradient cases exhibit the same self-similar property $a_{m_{i}}(r)=a_{m_{i+1}}(r \varphi)$. Furthermore, in Figure 20 we present the trajectories of wavevectors for the active set of modes. Not only are they nearly identical in both cases, they also exhibit a self-similar property in that each of the active modes follows the same path. The points at which the wavevectors cross the $k_{0}$ (dashed) circle correspond to hexagonal configurations. We return to these properties in more detail later.

These findings establish, if not a formal equivalence, at least an empirical equivalence between the gradient and nongradient forms of (27).

Result 2. Figures 21 to 23 In Figure 22, we show that the dominant amplitudes over the range $13<$ $r<89$ for the first two simulations belong to those modes generated by quadratic nonlinear interactions, starting from the initial parastichy wavevectors $\left(\boldsymbol{k}_{89}, \boldsymbol{k}_{144}\right)$ and $\left(\boldsymbol{k}_{76}, \boldsymbol{k}_{133}\right)$ respectively, which follow from the Fibonacci rule $\boldsymbol{k}_{144}-\boldsymbol{k}_{89}=\boldsymbol{k}_{55}$ and thereafter $\boldsymbol{k}_{34}, \boldsymbol{k}_{21}, \boldsymbol{k}_{13}$ (resp. $\boldsymbol{k}_{47}, \boldsymbol{k}_{29}, \boldsymbol{k}_{18}$ ). The amplitudes of modes generated by other binary combinations (e.g. $\boldsymbol{k}_{29}=\boldsymbol{k}_{8}+\boldsymbol{k}_{21}, \boldsymbol{k}_{42}=2 \boldsymbol{k}_{21}$ ) are much smaller. In Figure 23 . we plot the amplitude of each mode generated by the Fibonacci rule as a function of $r$. To illustrate the self-similar property, we plot $a_{m_{j}}$ as a function of $r / \varphi^{j}$. All amplitudes lie on the same curve. Moreover, the amplitudes of all the modes generated by the Fibonacci rule of the second simulation, $a_{123}, a_{76}, a_{47}, a_{29}, a_{18}$, $a_{11}, a_{7}, a_{4}, a_{3}, a_{1}$ lie on the same curve except it is phase shifted by a calculable amount. If $\left(m_{1}, m_{2}\right)$ and $\left(n_{1}, n_{2}\right)$, here $(1,2)$ and $(1,3)$, are the first two members in two different Fibonacci sequences, it is easy to show [49] that the ratio of corresponding numbers $n_{j} / m_{j}$ tends to $\varphi_{m n}=\frac{n_{1}+\varphi n_{2}}{m_{1}+\varphi m_{2}}$ which here is $\frac{1+3 \varphi}{1+2 \varphi} \approx 1.4$. Thus $a_{m_{j}}\left(r / \varphi^{j}\right)=a_{n_{j}}\left(r \varphi_{m n} / \varphi^{j}\right)$.

What is very clear from this analysis is that the Fibonacci rule dominates the dynamics of mode selection in the evolution of solutions of (27).

Result 3. Figure 24. In Figure 24 we show the paths of the radial wavenumbers by graphing $\mp \ell_{m_{j+1}}(r), \pm \ell_{m_{j}}(r)$ in the $\mp \ell_{m_{j+1}}, \pm \ell_{m_{j}}$ plane. All radial wavenumbers follow the same path. They each have the self-similar property that $-\ell_{m_{j+1}}(r \varphi)=\ell_{m_{j}}(r)$.

Result 4. Figure 25. By using the transformations $(25)$ and $(26)$ to convert the wavevectors with locally dominant amplitudes into the local rise and divergence angle coordinates, we plot for both the $(1,2)$ and $(1,3)$ initiated Fibonacci sequences the corresponding curves overlaid on the appropriate section of Figure 6 . We note that we obtain nearly identical results in the gradient case and the nongradient case.

Result 5. Figure 26. Why Fibonacci? We begin with Figure 26 which plots the motion in the $(\ell, m / r)$ plane of the wavevectors of the dominant modes corresponding to the Fibonacci branch joining the hexagons $(5,8,13)$ and $(8,13,21)$ in the gradient case. The wavenumber locus $\ell^{2}+m^{2} / r^{2}=k_{0}^{2}$ is drawn with a dashed semicircle. The preferred wavenumber $k_{0}$ is close to, but a nonlinear correction of, the linear preferred wavenumber unity. A formula is given in Section 3.3 . At $r=11.820$, the wavevectors lie on the circle and are separated by $60^{\circ}$ angles. Clearly the resolvent $\boldsymbol{k}_{5}+\boldsymbol{k}_{8}=\boldsymbol{k}_{13}$ lies on $\left|\boldsymbol{k}_{13}\right|=k_{0}$. As $r$ increases, the wavevectors $\boldsymbol{k}_{13}$ and $\boldsymbol{k}_{8}$, the parastichies in the rhombic region, continue to lie on the circle but move down, to the left and right respectively. The wavevector $\boldsymbol{k}_{5}$ arising from the binary composition $\boldsymbol{k}_{5}=\boldsymbol{k}_{13}-\boldsymbol{k}_{8}$ is pushed away from the preferred circle and its amplitude diminishes. The irregular Fibonacci addition $\boldsymbol{k}_{5}+\boldsymbol{k}_{13}=\boldsymbol{k}_{18}$, not shown here, can be seen by observation to be very far from the preferred circle because the angle between the $\boldsymbol{k}_{5}$ and $\boldsymbol{k}_{13}$ legs decreases and the length of $\boldsymbol{k}_{5}$ increases. But the Fibonacci addition wavevector, $\boldsymbol{k}_{21}, \boldsymbol{k}_{8}+\boldsymbol{k}_{13}=\boldsymbol{k}_{21}$, is drawn in towards the circle. Its amplitude increases. When we reach $r=19.105=11.820 \varphi$, the $\boldsymbol{k}_{21}$ mode has reached the circle and again the three modes $\boldsymbol{k}_{8}, \boldsymbol{k}_{13}$ and $\boldsymbol{k}_{21}$ have length $k_{0}$ and are $60^{\circ}$ apart in angle, a new hexagonal configuration. One can see that the "opposed" property is very important. When alternate wavevectors have radial wavenumbers with opposite signs $\left(\ell_{5}, \ell_{13}\right.$ are negative, $\ell_{8}$ positive), then, as $r$ increases, the legs of sequential wavevectors (here $\boldsymbol{k}_{8}$ and $\boldsymbol{k}_{13}$ ) open, drawing in their resolvent $\boldsymbol{k}_{21}=\boldsymbol{k}_{8}+\boldsymbol{k}_{13}$ towards the preferred circle whereas the resolvent corresponding to the irregular addition of every other mode (here $\boldsymbol{k}_{18}=\boldsymbol{k}_{5}+\boldsymbol{k}_{13}$ ) gets pushed further away. We obtain nearly identical results in the gradient case and the nongradient case.

Result 6 . One may think of the simulation of the solution starting with the parastichy numbers $(1,3)$ at the inner boundary as being analogous to the irregular Fibonacci addition branch of the simulation beginning with $(1,2)$. If we begin from the $(1,2,3)$ hexagonal configuration, the continuum of energy minima will follow branches joining successive triads in the regular Fibonacci sequence $1,2,3,5,8,13, \ldots$ On the other hand, for a radius value slightly greater than the radius value at which the $1,2,3$ hexagon dominates, a new continuous locus of minima $1,3,4,7,11,18,29, \ldots$ will emerge. They will arise initially from the irregular Fibonacci addition $1+3=4$ but thereafter the pattern will follow a continuous path determined by regular 
Fibonacci addition.

Result 7. Figures 27 to 29. The local front speed $\nu(r)$, packing efficiency $\eta(r)$, and energy $\epsilon(r)$ are drawn as a function of $r$. Each satisfies the $\log$ periodic property that $\nu(r \varphi)=\nu(r), \eta(r \varphi)=\eta(r), \epsilon(r \varphi)=\epsilon(r)$. The front speed is maximum at the hexagon regions and minimum in the rhombic regions. In contrast, the annular energy $\epsilon(r)$ is minimum in the hexagonal region and maximum in the rhombic regions. The packing efficiency $\eta(r)$, as one might expect from the result on planes, is maximum in the hexagonal region and minimum in the rhombic region.

Result 8. Figure 30. In Figure 30, we plot contours of the front speed $\nu$ as a function of the model parameters, $\mu$ the amount by which reverse diffusion in the auxin model overcomes regular diffusion and $\beta$ essentially measuring a product of PIN1 transport and average auxin concentration. The front speed is measured by initiating a $(1,2,3)$ hexagonal pattern on a cylinder which has a radius for which this pattern fits, taking into account the nonlinear correction to the energetically preferred wavenumber $k_{0}$. The front is allowed to propagate under 23) for 2000 time units, and the average front speed is computed. From the contour plot, it is straightforward to determine the values of $\mu$ and $\beta$ for which the front is pushed or pulled. For pulled fronts, the speed should not depend on the nonlinear coupling $\beta$, as pulled fronts are organized by conditions ahead of the front where the field amplitudes are small. We observe this in the figure for $\mu>0.1$ since the contour lines are vertical. In this region, we observe that $\nu \propto \sqrt{\mu}$. For smaller values of $\mu$, the lines are vertical for small $\beta$, but eventually, as $\beta$ increases, the contour lines deviate. This indicates that the nonlinearity is influencing the speed and other characteristics of the pattern, which are determined by conditions behind the front. Such fronts are pushed. Only in the pushed region can the three modes of the hexagonal region (or in the case of the rhombic region, combinations of sequential triads of modes) propagate in synchrony, a phase locking induced by nonlinear coupling.

For a discussion of front propagation in patterns, we refer the reader to the review article of van Saarloos [59] and references therein. Here we briefly indicate why it is that we must be in the pushed front regime. Pulled fronts have the property that their speeds are proportional to $\sqrt{\mu l^{2}}$, where $l$ is the radial wavenumber. Thus, in the pulled front regime of the $\mu, \beta$ plane, the different modes in the pattern will propagate at different speeds. Indeed, in the pulled front region, we can clearly see this mode separation in simulations. However, in the pushed front regime, the different modes participating in the Fibonacci pattern synchronize and travel together.

Result 9. Figure 31. In the gradient case, Fibonacci patterns can be thought of as long lived saddle points of (27) with strongly attracting stable manifolds and weakly repelling unstable ones. The reason is that on large disks, the local geometry looks planar and the rhombic regions cannot be held in place by their hexagonal neighbors but slowly develop defects in order to realize locally hexagonal patches. To investigate this behavior and in particular the time scales of the various dynamical behaviors we excise the $34<r<55$ annulus and study its dissipation rate

$$
-\frac{d \mathcal{E}}{d t}=-\int \frac{\delta \mathcal{E}}{\delta u} \frac{\partial u}{\partial t}=\int\left(\frac{\delta \mathcal{E}}{\delta u}\right)^{2}
$$

for 1000 time units over this annulus. The features of Figure 31 that we note are that the dissipation rate is large for approximately 100 units, thereafter followed by a sharp decrease to a level of one thousand times less at which level it stays almost constant for a further 600 time units, after which there are a few rapid spikes across each of which the energy drops by a series of discrete events. Specifically, for $t<50$, the pushed front is propagating through the annular domain, so the energy gradient is large and is concentrated about the transition region. Note that the dissipation rate fluctuates due to the difference in energy between hexagonal and rhombic regions; the maximum occurs when the front is propagating through the most hexagonal region. Once the front has left the domain, the dissipation rate falls by more than three orders of magnitude. For $50<t<300$, the dynamics are dominated by a very weakly unstable trajectory that does not change the overall planform. For $t>300$, global reorganization of the planform begins to occur. Rhombic regions develop into hexagonal patches separated by phase grain boundaries. The reorganization also opens up gaps in the planform which eventually become defects after the formation of a new maximum. These events appear as sharp peaks in the dissipation rate starting at $t \approx 700$. The role of defects is to convert the rhombic regions into local hexagonal patches with different orientations separated by line and point defects. Subsequent coarsening of these patches occurs on very, very long time scales. 
We make two observations regarding these results. First, the time scale of global reorganization is much longer than the time that it takes for the pattern to be fully laid down by the front. The Fibonacci pattern is therefore a saddle point in the energy landscape more closely resembling a canyon. The front provides a mechanism for the system to rapidly reduce its energy, with the price being a bias imposed by the pattern already laid down in the vicinity of the transition region. Second, it is not unreasonable to expect that there is a change in the mechanism of morphogenesis once a primordium has been initiated. In particular, there may be a way for young primordia to fix their position on the plant. Our results indicate that this process would not need to occur immediately after formation. It would only need to be faster than the global reorganization time scale in order to preserve the planform.

\subsection{Derivation of the amplitude or order parameter equations}

Recall that $\mu$ in $(23)$, when the original equations were rescaled, is the amount by which reverse diffusion of the auxin fluctuation field $u(r, \theta, t)$ overcomes normal diffusion. For $\mu<0$, the solution $u=0$ is linearly (not necessarily nonlinearly) stable. For $\mu>0$, certain shapes and configurations

$$
H_{m}^{ \pm}\left(k_{m} r\right) e^{i m \theta}
$$

will grow in time. Here $H_{m}^{ \pm}\left(k_{m} r\right)$ is a solution of the Hankel equation $y^{\prime \prime}+\frac{1}{x} y^{\prime}-\frac{m^{2}}{x^{2}} y=-y, x=k_{m} r$. The growth rate $\sigma_{m}$ is given by

$$
\sigma_{m}=\mu-\left(k_{m}^{2}-1\right)^{2} .
$$

The parameter $k_{m}$ arises from the action of the Laplacian $\nabla^{2}$ on $H^{ \pm}\left(k_{m} r\right) e^{i m \theta}$ to give $-k_{m}^{2} H_{m}^{ \pm}\left(k_{m} r\right) e^{i m \theta}$. It is what we call the wavenumber. For $k_{m}^{2}=1$, we see the shapes 30 begin to grow exponentially. They will then interact with each other via the nonlinear terms, both quadratic and cubic, in (23) and certain combinations of the modes will outcompete others. The winner of this competition will be the chosen planform.

We start with an appropriate and soon-to-be-defined linear combination of the modes (30). We call this $u_{0}$. Then we expand the full solution $u(r, \theta, t)$ as an asymptotic expansion

$$
u(r, \theta, t)=u_{0}(r, \theta, t)+u_{1}(r, \theta, t)+\cdots
$$

in such a way that the ratio of $u_{1}$ to $u_{0}$ remains (uniformly in $r, t$ ) small as the size of the parameter $\mu$ becomes small. The iterates in $32, u_{1}, \ldots$, will satisfy equations of the form

$$
\left(\nabla^{2}+1\right)^{2} u_{1}=\text { RHS }
$$

where the RHS of 33 will contain all terms generated by the nonlinear interactions of the shapes $\left\{H_{m}^{ \pm}\left(k_{m} r\right) e^{i m \theta}\right\}_{m}$ (we call these modes) contained in $u_{0}$. In most cases, the solution of 33 will be bounded; namely for most combinations of quadratic and cubic products of the modes in $u_{0}$, the wavenumbers of modes generated in this way will not be close to unity. However, in some cases, namely when the wavenumbers of terms arising on the RHS of (33) are close to unity, the corresponding solution $u_{1}$ will have a small or zero denominator because the operator $\nabla^{2}+1$ will be small. It is unacceptable to leave these in $u_{1}$ because then the validity of the asymptotic expansion (32) is compromised. We remove these terms by allowing the amplitudes which multiply the modes contained in $u_{0}$ to be slowly varying in time and choose that time variation so that we eliminate all terms in $u_{1}$ which have small or zero denominators. This choice leads to what we call the amplitude equations.

The first task and the first choice we have to make is to decide which modes to include in $u_{0}(r, \theta, t)$. To make things slightly easier, with almost no compromise of accuracy, we will approximate $H_{m}^{ \pm}\left(k_{m} r\right)$ by its asymptotic shape $\exp \pm i \int \ell_{m} d r$ where $\ell_{m}^{2}=k_{m}^{2}-\frac{m^{2}}{r^{2}}$. This is valid when both $m$ and $r$ are large and of the same order, and the radius $r$ of the disk is large compared with the pattern wavenumber. In this approximation, $\frac{\partial^{2}}{\partial r^{2}} u_{0} \gg \frac{1}{r} \frac{\partial u_{0}}{\partial r}$, the Laplacian $\nabla^{2}$ is $\frac{\partial^{2}}{\partial r^{2}}+\frac{1}{r^{2}} \frac{\partial^{2}}{\partial \theta^{2}}$ and the wavenumber $k_{m}^{2}=\ell_{m}^{2}+\frac{m^{2}}{r^{2}}$. Then,

$$
u_{0}(r, \theta, t)=\sum_{m \in S} A_{m}(r) \exp \left(i \int \ell_{m}(r) d r+i m \theta\right)+(*)
$$


where we will allow $\ell_{m}(r)= \pm \sqrt{k_{m}^{2}-m^{2} / r^{2}}$ to have either sign. We suppress the $t$ dependence in writing (34). The set $S$ will contain all modes with wavevector $\boldsymbol{k}_{m}=\left(\ell_{m}= \pm \sqrt{k_{m}^{2}-m^{2} / r^{2}}, \frac{m}{r}\right)$ whose wavenumber $k_{m}$ is close to unity because it is these modes (see (30)) which will either grow, remain neutral or be very weakly damped in the linear approximation. They therefore live long enough to interact nonlinearly. It will turn out that the preferred mode wavenumber $k_{0}$ is slightly different to unity because of a nonlinear correction. Therefore the set $S$ contains all those modes whose wavenumbers $k_{m}$ are within a certain distance of $k_{0}$, namely all those wavevectors which lie in the annulus

$$
k_{0}-K<\left|\boldsymbol{k}_{m}\right|<k_{0}+K
$$

at any radius $r$ that the pattern occupies.

Our results are independent of the choice of $K$ for sufficiently large $K$. Because we numerically solve the amplitude equations, in practice we take $K$ large and include in $S$ many more modes than is necessary. The set of amplitudes $A_{m}$ contained in $S$ are called order parameters. They are the amplitudes of what are called the active modes, namely the set $\mathbf{A}$ of those modes which dominate the pattern field at some $r$.

The second task is to examine the terms produced by quadratic and cubic products of the active modes. Let us first look at the quadratic product of two modes in $u_{0}$, which we write as shorthand $e^{i \boldsymbol{k}_{r} \cdot \boldsymbol{x}}$ and $e^{i \boldsymbol{k}_{s} \cdot \boldsymbol{x}}$ where $\boldsymbol{k}_{j} \cdot \boldsymbol{x}$ stands for $\int \ell_{j} d r+m_{j} \theta, j=r, s$. The product will give the term $e^{i\left(\boldsymbol{k}_{r}+\boldsymbol{k}_{s}\right) \cdot \boldsymbol{x}}$. For most binary pairings of modes $\boldsymbol{k}_{j}$ which belong to $\mathbf{A}$ and whose wavenumbers lie close to $k_{0}$, the vector sum resolvent $\boldsymbol{k}_{r}+\boldsymbol{k}_{s}$ will have a wavenumber far different from $k_{0}$. Therefore when we solve $\left(\nabla^{2}+1\right)^{2} u_{1}=e^{i\left(\boldsymbol{k}_{r}+\boldsymbol{k}_{s}\right) \cdot \boldsymbol{x}}$, the denominator of the solution is not small. Such a mode (call its amplitude $A_{r, s}$ ) will depend algebraically on the product $A_{r} A_{s}$ and will be small in amplitude. We include all such modes in what we call the passive set $\mathbf{P}$. The amplitudes of all modes in the passive set are simple algebraic functions of the modes in the active set given by the graph $\mathbf{P}=\mathbf{P}(\mathbf{A})$, called the center manifold. The phase point representing the state of the system, coordinatized by the amplitudes of all modes, very quickly relaxes onto the center manifold $\mathbf{P}=\mathbf{P}(\mathbf{A})$. Once on the center manifold, the phase point relaxes slowly towards any fixed points or, in some cases, other attractors on this manifold. The slow motion on the center manifold is given by the amplitude equations which we now derive.

For pairings where the resolvent wavevector $\boldsymbol{k}_{r}+\boldsymbol{k}_{s}$ lies within the annulus (35), the denominator in $u_{1}$ can be small and the result is that the asymptotic expansion $(32)$ is no longer valid. We remove these terms by including that combination $\boldsymbol{k}_{r}+\boldsymbol{k}_{s}$ in $u_{0}$ (let us call it $\boldsymbol{k}_{m}$ ) and allowing its corresponding amplitude to be a slowly varying function of time. This adds to the RHS of 33 an extra term $-\frac{\partial}{\partial t} A_{m} e^{i\left(\boldsymbol{k}_{r}+\boldsymbol{k}_{s}\right) \cdot \boldsymbol{x}}=$ $-\frac{\partial A_{m}}{\partial t} e^{i \boldsymbol{k}_{m} \cdot \boldsymbol{x}}$, and we choose $\frac{\partial A_{m}}{\partial t}$ to eliminate the small denominator (secular) term in $u_{1}$.

For example, if we choose the two modes $A_{r} e^{i \boldsymbol{k}_{r} \cdot \boldsymbol{x}}, A_{s} e^{i \boldsymbol{k}_{s} \cdot \boldsymbol{x}}$ and compute $-\beta \nabla\left(u_{0} \nabla u_{0}\right)$ where $\boldsymbol{k}_{m}=$ $\boldsymbol{k}_{r}+\boldsymbol{k}_{s}$, we will obtain $\beta k_{m}^{2} A_{r} A_{s} e^{i \boldsymbol{k}_{m} \cdot \boldsymbol{x}}$. From the cubic product, we will obtain the coefficient of $e^{i \boldsymbol{k}_{m} \cdot \boldsymbol{x}}$ to be $3 A_{m}\left(\left|A_{m}\right|^{2}+2 \sum_{r \neq m}\left|A_{r}\right|^{2}\right)$. Then the choice of $\frac{\partial A_{m}}{\partial t}$ is

$$
\frac{\partial A_{m}}{\partial t}=\left(\mu-\left(k_{m}^{2}-1\right)^{2}\right) A_{m}+\sum_{\boldsymbol{k}_{m}=\boldsymbol{k}_{r}+\boldsymbol{k}_{s}} \tau_{-m r s} A_{r} A_{s}-3 A_{m}\left(\left|A_{m}\right|^{2}+2 \sum\left|A_{r}\right|^{2}\right)
$$

where $\tau_{-m r s}=\beta k_{m}^{2}$. The first sum is taken over all $r, s$ modes for which $\boldsymbol{k}_{r}+\boldsymbol{k}_{s}=\boldsymbol{k}_{m}$. The second sum is over all active modes except for $\boldsymbol{k}_{m}$. The $\left(k_{m}^{2}-1\right)^{2}$ linear term arises from the fact that $\left(\nabla^{2}+1\right)^{2} u_{0}$ will have a small contribution. Equations (36), one for each $\boldsymbol{k}_{m}$ in $S$, are the amplitude equations. If all wavevectors $\boldsymbol{k}_{m}$ in the active set were nearly equal, $\tau_{-m r s}$ would be symmetric over all indices $-m, r, s$. Then, (36) could be written as a gradient flow

$$
\frac{\partial A_{m}}{\partial t}=-\frac{\partial E}{\partial A_{m}^{*}}
$$

where

$$
\begin{gathered}
E=-\sum\left(\mu-\left(k_{m}^{2}-1\right)^{2}\right) A_{m} A_{m}^{*}-\sum_{m, r, s}\left(\tau_{-m r s} A_{m}^{*} A_{r} A_{s}+\right. \\
\left.\tau_{m-r-s} A_{m} A_{s}^{*} A_{s}^{*}\right)+\frac{3}{2}\left(\sum_{m} A_{m}^{*} A_{m}^{* 2}+4 \sum_{r \neq m} A_{m} A_{m}^{*} A_{r} A_{s}^{*}\right)
\end{gathered}
$$


But, in our case, things are not that simple. In a planar geometry, where the active modes have wavevectors $\boldsymbol{k}_{m}$ close to unity and independent of spatial coordinates, the quadratic coupling coefficients $\tau_{-m r s}$, namely $\beta k_{m}^{2}$, would be all nearly equal, and the amplitude equations for 23), near onset, would be all nearly equal even though the pde 223 is not. For us here, however, the mode with wavevector $\boldsymbol{k}_{m}$ will only have wavenumber close to $k_{0}$ or unity in a certain range of radius, namely that interval in $r$ where $m$ is one of the parastichies. Consider the interval $R_{m}<r<R_{n}=R_{m} \phi$, where the modes with wavevectors $\boldsymbol{k}_{n-m}, \boldsymbol{k}_{m}, \boldsymbol{k}_{n}$ have almost equal wavenumbers and amplitudes at $R_{m}$, and the pattern is hexagonal, and the modes with wavevectors $\boldsymbol{k}_{m}, \boldsymbol{k}_{n}, \boldsymbol{k}_{m+n}$ have the same property at $R_{n}$ and again the pattern is hexagonal. At $r=R_{m}, R_{n}$, the amplitude equations for the active modes are gradient. For values in between, they are not because, as $r$ moves between $R_{m}$ and $R_{n}$, the wavenumber $k_{n-m}\left(k_{m+n}\right)$ goes from $k_{0}\left(\sqrt{3} k_{0}\right)$ to $\sqrt{3} k_{0}\left(k_{0}\right)$. Moreover, in the next interval $R_{n}=R_{m} \phi$ to $R_{m+n}=R_{n} \phi$, the wavevector $\boldsymbol{k}_{n-m}$ will have moved away from the circle $|\boldsymbol{k}|=k_{0}$ altogether, and the quartet $\boldsymbol{k}_{m}, \boldsymbol{k}_{n}, \boldsymbol{k}_{m+n}, \boldsymbol{k}_{m+2 n}$ will be the next set of most active modes. Therefore for phyllotactic patterns, and indeed for any similar system where the basic geometry is, or is similar to, a disk on which the pattern forms annulus-by-annulus, the set of active modes is not fixed, but rather changes with radius. The active modes, to borrow a line from Shakespeare, have their exits and their entrances. They are active at some range of $r$ values, but passive (namely, their amplitudes are determined by algebraic dependencies on the current active modes) at all others. What we have already seen, however, from our simulation results in Section 3.2, is that all modes with wavevectors $\boldsymbol{k}_{m}$ which are active at some stage have subscript integers determined by the Fibonacci rule. Modes with wavevectors such as $\boldsymbol{k}_{2 m+n}=\boldsymbol{k}_{m}+\boldsymbol{k}_{m+n}$ which are generated by the quadratic nonlinear terms not consistent with the Fibonacci rule are always passive and never active.

In phyllotactic geometries, where there are at most three active modes at each $r$ in the interhexagon range, the center manifold $P(A)$ is the surface in the space coordinated by all amplitudes for which the passive amplitudes are expressed as algebraic functions of the amplitudes which happen to be active in that range. Because of the self-similar properties of the developing pattern, the graph $P=P(A)$ in one range is equivalent, under the self-similar property enjoyed by the sets $\left\{A_{m}, l_{m}\right\}$, to its counterpart in the neighboring range. Instead of the center manifold's being constant, it is constant up to some scaling equivalence.

We conclude this section with a discussion of four significant issues: (i) Why it is, even though $\tau_{-m r s}$ is not symmetric in all indices at each $r$, that the overall pattern behaves as if (36) is, to a good approximation a gradient flow; (ii) How the preferred wavenumber $k_{0}$, which is close to unity, might be chosen precisely; (iii) How modes not generated by the Fibonacci rule can arise as instabilities and interrupt the development of a Fibonacci pattern; (iv) The role of whorls and their interactions with Fibonacci patterns.

In Section 3.1, we introduced equation (28) which we designated as companion to (23) and which was gradient with a free energy given by $(29)$. It originated by writing the quadratic nonlinearity $-\beta \nabla(u \nabla u)$ in (23) as

$$
-\beta \nabla(u \nabla u)=-\frac{\beta}{3}\left(2 u \nabla^{2} u+(\nabla u)^{2}\right)-\frac{\beta}{3}\left(u \nabla^{2} u+2(\nabla u)^{2}\right) .
$$

The first term is gradient with energy given by (38) with $\gamma=0$ and with coupling coefficient $\tau_{-m r s}=$ $\frac{2 \beta}{3}\left(\boldsymbol{k}_{r} \cdot \boldsymbol{k}_{s}-\boldsymbol{k}_{m} \cdot \boldsymbol{k}_{s}-\boldsymbol{k}_{m} \cdot \boldsymbol{k}_{r}\right)$ which is symmetric under the interchange of any of the indices $-m, r, s$. At values of $r$ where the pattern is hexagonal, it is equal to $\beta k_{0}^{2}$. The second term has quadratic coefficient $\frac{\beta}{3}\left(k_{r}^{2}+k_{s}^{2}+\boldsymbol{k}_{r} \cdot \boldsymbol{k}_{s}\right), \boldsymbol{k}_{m}=\boldsymbol{k}_{r}+\boldsymbol{k}_{s}$. It is not symmetric, but is zero at hexagonal radii. However, the simulations in Section 3.2 display very little difference between the patterns produced by (23) and its companion gradient flow (28). Why is it that in the rhombic regions in between the hexagonal radii, the difference does not seem to matter? Consider a value of $r$ in the middle of the interval $\left(R_{m}, R_{n}\right)$. The two wavenumbers $k_{n-m}$ and $k_{m+n}$ are now far from the preferred values $k_{0}$ although the parastichy numbers remain close (see Figure 20). Therefore, the coefficients of the linear terms in the amplitude equations for $A_{n-m}$ and $A_{m+n}$ are large, and the resulting small amplitudes $A_{n-m} A_{m+n}$ are given, to a good approximation, by a small coefficient times the product $A_{m}^{*} A_{n}$, respectively $A_{m} A_{n}$. Resubstituting these expressions into the equations for parastichy amplitudes $A_{m}, A_{n}$ leads to equations which contain only linear and cubic terms. The quadratic terms have been eliminated. The equations are again gradient, with free energy $E\left(A_{m}, A_{n} ; \boldsymbol{k}_{m}, \boldsymbol{k}_{n}\right)$ containing only quadratic and quartic terms. This argument is, however, only an indicator as to why the gradient character of 23) obtains. It does not address what happens for $r$ approaching $R_{m}, R_{n}$, where $k_{n-m}$ and $k_{m+n}$ are close to $k_{0}$, although we know that at $R_{m}$ and $R_{n}$, the amplitude equations are gradient.

We now address question (ii). What chooses the preferred wavenumber? For the companion gradient 
flow (28), with free energy $E$ given by $(29)$, the answer is straightforward. We simply minimize $E$ with respect to both amplitudes $\left\{A_{m}\right\}$ and the two wavenumbers $k_{m}=\sqrt{l_{m}^{2}+m^{2} / r^{2}}, k_{n}=\sqrt{l_{n}^{2}+n^{2} / r^{2}}$, whose wavevectors $\boldsymbol{k}_{m}, \boldsymbol{k}_{n}$ generate all modes arising from nonlinear interactions. In Figure 20, we show the path of the parastichy wavevectors in $(l, m / r)$-space. In the interval $\left(R_{m}, R_{m+n}\right)$, the universal path has three intersections with $|\boldsymbol{k}|=k_{0}$ which correspond to those values of $r$ at which the pattern is dominated by hexagons with wavevectors $\left(\boldsymbol{k}_{n-m}, \boldsymbol{k}_{m}, \boldsymbol{k}_{n}\right),\left(\boldsymbol{k}_{m}, \boldsymbol{k}_{n}, \boldsymbol{k}_{m+n}\right)$, and $\left(\boldsymbol{k}_{n}, \boldsymbol{k}_{m+n}, \boldsymbol{k}_{m+2 n}\right)$. Using Mathematica, the value of $k_{0}$ is found to be $\sqrt{\frac{270-\beta^{2}+\beta \sqrt{\beta^{2}(9-8 \mu)+540 \mu}}{270-4 \beta^{2}}}$. For values of $r$ in between $R_{m}, R_{n}$ and $R_{n}, R_{m+n}$, the energetically chosen wavenumber $k_{p}(r)$ for the parastichy modes $\boldsymbol{k}_{m}, \boldsymbol{k}_{n}$ and $\boldsymbol{k}_{n}, \boldsymbol{k}_{m+n}$ respectively, is close to, but slightly less than $k_{0}$, and closer to unity, the linear value.

For the nongradient system, the amplitude equations for (23), there is no obvious preferred wavenumber selection rule. Experience with other pattern-forming systems teaches us that the wavenumbers selected by curved rolls $k_{c}$, by stationary dislocations $k_{d}$, by boundaries $k_{b}$, are all equal for gradient flows (and equal to $k_{0}$ ) but not equal for non gradient flows. Near onset, they are close to the linear preferred wavenumber. What we have done, therefore, is to choose the wavenumber $k_{p}(r)$ determined by the companion gradient flow (28) to (23) as the preferred wavenumber when solving the stationary amplitude equations of (23) for the amplitudes $\left\{A_{m}\right\}$. Our guess is that the selection is made by a condition connected with the front propagation whose velocity $\nu$ depends on this choice. Note that we have shown that the velocity $\nu(r)$ does change with $r$. It is slightly faster at hexagons (where the preferred wavenumber is $k_{0}$ ) than it is in the rhombic regions (where $k_{p}(r)$ is close to, but slightly less than, $k_{0}$ ).

We next address question (iii) as to how modes not generated by the Fibonacci addition rule can arise via instabilities and interrupt Fibonacci patterns. Let us take a concrete example which we illustrate again in Section 3.5 using direct simulation. We follow the evolution of the Fibonacci pattern from a hexagonal structure with parastichies $3,5,8, \boldsymbol{k}_{3}+\boldsymbol{k}_{5}=\boldsymbol{k}_{8}$ to a hexagonal structure $2,3,5, \boldsymbol{k}_{2}+\boldsymbol{k}_{3}=\boldsymbol{k}_{5}$. The role of the common circumferential wavenumber 3 is key. We ask: Given that we can also have the quadratic interaction $\boldsymbol{k}_{3}+\boldsymbol{k}_{3}^{\prime}=\boldsymbol{k}_{6}$, when is it possible for the modes $3^{\prime}$ and 6 to emerge from an instability. Here, we denote by $3^{\prime}$ a second mode with the circumferential wavenumber 3 but a radial wavenumber of opposite sign. Consider the amplitude equations for $A_{3}^{\prime}$ and $A_{6}$.

$$
\begin{aligned}
\frac{d A_{3}^{\prime}}{d t} & =\widetilde{\sigma}_{3}^{\prime} A_{3}^{\prime}+3 \beta\left(k_{3}^{\prime}\right)^{2} A_{6} A_{3}^{*} \\
\frac{d A_{6}}{d t} & =\widetilde{\sigma}_{6} A_{6}+3 \beta\left(k_{6}\right)^{2} A_{3} A_{3}^{\prime}
\end{aligned}
$$

where we have included the cubic terms in $\widetilde{\sigma}_{3}^{\prime}$ and $\widetilde{\sigma}_{6}$; namely $\widetilde{\sigma}_{3}^{\prime}=\sigma_{3}^{\prime}-\left(\left|A_{2}\right|^{2}+\left|A_{3}\right|^{2}+\left|A_{5}\right|^{2}+\left|A_{8}\right|^{2}\right)$. We check the growth rate of $A_{3}^{\prime}, A_{6}$ by writing

$$
\left(\frac{d}{d t}-\tilde{\sigma}_{3}^{\prime}\right)\left(\frac{d}{d t}-\widetilde{\sigma}_{6}\right) A_{6}=9 \beta^{2}\left(k_{3}^{\prime}\right)^{2}\left(k_{6}\right)^{2}\left|A_{3}\right|^{2} A_{6} .
$$

The growth rate $\sigma$ of $A_{6}$ is given by

$$
\left(\sigma-\widetilde{\sigma}_{3}^{\prime}\right)\left(\sigma-\widetilde{\sigma}_{6}\right)=9 \beta^{2}\left(k_{3}^{\prime}\right)^{2}\left(k_{6}\right)^{2}\left|A_{3}\right|^{2}
$$

and can be positive. The question is then if this growth rate $\sigma$ is large enough so that the instability can grow in the time it takes for the front leaving the pattern $3,5,8$ to reach the pattern $2,3,5$. Let $r_{0}$ be the radius at which the $2,3,5$ pattern is exactly hexagonal. Then $r_{0} \varphi, \varphi=\frac{\sqrt{5}+1}{2}$, is the radius at which the pattern $3,5,8$ is exactly hexagonal. Let the average front speed be $c$. Then the transition time is $\frac{c}{r_{0}(\varphi-1)}$. If this is small compared to $1 / \sigma$, then the Fibonacci pattern will continue uninterrupted. If it is large or comparable, then the Fibonacci pattern will be interrupted and a whole new set of modes will enter the dynamics.

We illustrate both scenarios in Section 3.5 where we mimic the transition by the length of the intermediate region between two cylinder of radii $r_{0} \varphi$ and $r_{0}$. For quick transitions, the pattern remains Fibonacci. For slow ones, it does not.

Such instabilities are analogous to the Eckhaus and zig-zag instabilities of roll patterns in planar geometries. For example, the zig-zag instability occurs when the wavenumber $k_{0}-\kappa$ of a roll pattern is less than 
the preferred wavenumber $k_{0}$. It is unstable to modes with wavevectors $\boldsymbol{k}_{ \pm}=\left(k_{0}-\kappa, \pm \sqrt{2 k_{0} \kappa-\kappa^{2}}\right.$, each of whose wavenumbers is $k_{0}$ via the cubic interaction $\boldsymbol{k}_{+}+\boldsymbol{k}_{-}=2\left(k_{0}-\kappa, 0\right)$. The coupled equations for the respective amplitudes $A_{+}$and $A_{-}$will contain terms proportional to $A_{-}^{*} A_{0}^{2}$ and $A_{+}^{*} A_{0}^{2}$ respectively. $A_{+}$ and $A_{-}$will grow exponentially if $\kappa>0$. For the analogous instability in hexagonal patterns, the quadratic interactions play a similar role.

Finally, we address question (iv), namely the role of whorls and their interactions with Fibonacci patterns. In the development of our amplitude analysis in this review, we have not included whorl solutions. An $m$ whorl will have the modal representation

$$
\operatorname{Re}\left(A_{+} \exp \left(i \int \ell(r)+i m \theta\right)+A_{-} \exp \left(-i \int \ell(r)+i m \theta\right)+A_{0} \exp (2 i m \theta)\right)
$$

namely the superposition of identical spirals with opposite chiralities and a purely circumferential mode. When they are included, what we find is:

(a) For the intervals in $r$ for which they exist, they give rise to the lowest of all possible energy minima (see [53]).

(b) Outside these intervals, they either fail to exist or destabilize to a mode with circumferential wavenumbers $m, m+1,2 m+1$ which breaks the chiral symmetry. In some instances, the transition can be directly to the whorl $m+1, m+1,2 m+2$. We will include examples of these transitions in direct simulation case studies given in Section 3.5. In particular, we note these are plants which interpolate their phyllotactic configuration between whorl and Fibonacci patterns.

(c) While Fibonacci patterns can exist at all values of the generative annulus radius, whorls only exist in finite ranges. When the radius is fairly small, $3<r<8$, the ranges of existence tend to overlap so that whorl-to-whorl transitions can happen although they often do so via an intermediate spiral pattern (See case study 4 and Figure $40 \mathrm{~b}$ in Section 3.5).

(d) 2-Whorls can initiate many plant patterns because of a built in bias (cotyledon) in the meristem structure. A transition to a $(2,3,5)$ Fibonacci pattern then gives the sequence $1,2,3,5,8, \ldots$ an advantage over other sequences generated by the Fibonacci rule.

(e) Observations of leaf structures growing on the twigs of bushes and trees most often demonstrate low parastichy spiral patterns or low level 2 and 3 whorls. Since the parastichy number is principally determined by the radius of the generative annulus on which the phyllum is first formed, this means that in these situations the radius of the generative annulus remains more or less constant even though the plant itself continues to grow.

\subsection{Results from the amplitude equation analysis}

The striking feature of the results presented in this section is that they match precisely the results obtained from the simulation of the full pde when those patterns are Fourier analyzed. Namely, when the pattern is a Fibonacci progression, a smooth continuum of hexagons with circumferential wavenumbers $(n-m, m, n)$ with equal amplitudes $A_{n-m}, A_{m}, A_{n}$ and wavenumbers $k_{n-m}, k_{m}, k_{n}$, to hexagons with circumferential wavenumbers $m, n, m+n$ and amplitudes $A_{m}, A_{n}, A_{m+n}$, connected by regions of rhombic patterns in which there is a smooth transition between circumferential wavenumbers $n-m, m, n$ and $m, n, n+m$ with $A_{m+n}$ decreasing and $A_{n-m}$ increasing as $r$ decreases from $n$ to $m$, the amplitude equations give the same results as the pde simulation. In our calculations of solutions of the amplitude equations, we start at the outer boundary $r \simeq 233=89+144$ and end at $r \simeq 5=2+3$.

The amplitude analysis not only complements the simulations but adds additional understanding in three main aspects. First, the Fourier coordinates are best adapted for understanding phyllotactic patterns using the language of center manifold theory. Second, as we have said, this center manifold theory has new features very different from its manifestation in planar patterns. The amplitude equation approach suggests a new and exciting idea about the nature of that manifold. Third, in the gradient case, it reveals the presence of other free energy minima which have relevance for understanding the role of other branches in the Van Iterson diagram. 
We work with the sets of amplitude equations obtained for (27) with both $\gamma=0$ and $\gamma=1$. We solve the former set by minimizing the free energy $E$ obtained from (27) with $\gamma=0$ and written in (38) as a function of all the amplitudes $\left\{A_{m}\right\}$ and two radial wavenumbers. Recall the wavevectors of all participating modes are generated by starting with two (e.g. the dominant parastichies at either the outer or inner boundary) and taking all binary integer combinations. For example, if we start with $\boldsymbol{k}_{3}=\left(\ell_{3}, 3 / r\right)$ and $\boldsymbol{k}_{5}=\left(\ell_{5}, 5 / r\right)$, this set is $\left\{i \boldsymbol{k}_{3}+j \boldsymbol{k}_{5}\right\}$ where $i$ and $j$ are integers. We minimize $E$ with respect to all amplitudes as well as $\ell_{3}$ and $\ell_{5}$ (or any other pair of quadratically generated radial wavenumbers). Given $\ell_{3}, \ell_{5}$ and therefore all other radial wavenumbers $\ell_{m}$ (and therefore their corresponding wavenumbers $k_{m}$ ) minimizing $E$ with respect to the amplitudes is equivalent to solving the stationary amplitude equations for (27) in the case $\gamma=0$ given by (36). The amplitude equations for the nongradient case $\gamma=1$ are gradient only at that discrete set of radii where hexagons dominate. At other values of $r$, they are not. Therefore, since we do not have a free energy to minimize, we must have some other way of selecting the preferred wavenumber $k_{m}(r)$ in the nongradient case. In all likelihood it is selected by the properties of the front which ties the unstable $u=0$ state to the patterned state in its wake. Lloyd et al. 34] have studied stationary fronts which separate hexagonal and uniform states at the Maxwell point $\mu(\beta)<0$, but we do not know of any work which calculates the pushed front velocity as a function of the preferred wavenumber in the wake pattern or selects the wavenumbers in the case where $\mu>0$ or even in cases where $\mu<0$ when one state invades the other. What we do therefore is follow the results suggested by our simulations which show that the preferred wavenumbers of (27) in both the gradient and nongradient cases are the same at all $r$ (see Figure 20) and choose, when solving the stationary amplitude equations (36), the wavenumbers $k_{m}(r)$ given by the gradient flow. It is known that gradient flows select that wavenumber which is the same as that selected by other mechanisms.

In both cases, we end up with solutions $\left\{A_{m}(r), \ell_{m}(r), k_{m}(r)=\sqrt{\ell_{m}^{2}+m^{2} / r^{2}}\right\}_{m}$ for all $m$ participating modes. Since we solve the amplitude equations (36) and find the energy minima (38) numerically, we choose the $K$ in (35) to be large and include many more modes in the calculation than is necessary. Most of the modes are passive at all radii and, because their wavenumbers are far from the preferred wavenumber, their amplitudes will be very small. We express our findings in a series of results.

Result 1. We plot in Figure 32 the invariant amplitude curves for those modes in the Fibonacci progression which are active for some radius $r$ between 8 and 233. The results for the amplitude equations for both the gradient and nongradient flows are almost identical. At $r \approx 233$, the three amplitudes $A_{233}, A_{144}, A_{89}$ sit in left-to-right order on top of the amplitude invariant curve shown in Figure 32 As $r$ decreases, first the amplitude $A_{233}$ slides down the left part of the curve while $A_{55}$ climbs up the right side. At $r \approx 144$, $A_{144}, A_{89}, A_{55}$ occupy the same positions that $A_{233}, A_{144}, A_{89}$ did at $r \approx 233$. The leftward march of the amplitudes $A_{233}, A_{144}, A_{89}, A_{55}, A_{34}, A_{21}, A_{13}, A_{8}, A_{5}$ continues as the radius decreases all the way to $r \approx 8$. We note that at every $r$, the property $A_{m}(r)=A_{n}(r \varphi)$ holds, where $m<n$ are successive members of a Fibonacci sequence and $\varphi$ is the golden number.

Result 2. When the maximum amplitudes of the modes which are sometimes active or always passive in the interval $8<r<89$ are plotted, we obtain exactly the same results as shown in Figure 22. In all cases, they too have self-similar behavior; $A_{\text {current }}(r)=A_{\text {next }}(r \varphi)$. For example, in the interval $R_{m}, R_{n}$, both $A_{m+2 n}(r)=A_{2 m+3 n}(r \varphi)$ and $A_{2 m+n}(r)=A_{m+3 n}(r \varphi)$.

Result 3. The invariant radial wavenumber curve and the plot of the solution path in the $\left(\mp \ell_{m_{j}+1}, \pm \ell_{m_{j}}\right)$ plane are shown in Figures 33 and 34 . The wavevectors follow the same paths as calculated directly from simulations and which have already been shown in Figure20. Again we have self-similarity; $\ell_{m}(r)=-\ell_{n}(r \varphi)$.

These are remarkable results. All amplitudes and radial wavenumbers, not just the ones which are active at some $r$, but also those which are never active at any $r$, satisfy the self-similar properties. They are found to hold in the simulations of the full pde with initial data for initial data that have two dominant integer circumferential wavenumbers and are analytic consequences of the amplitude equations and, in the gradient case, corresponding free energy. For any two generating circumferential wavenumbers $m, n$, the set of allowed circumferential wavenumbers are all those generated by $i m+j n, i, j$ any integers which, if $m, n$ are coprime, is the set of all integers. What is remarkable is that there is little a priori reason to believe that solutions of the pde with these, admittedly special, initiating states should display all of these self similar properties. No obvious scaling of the pde leads to such a conclusion.

Result 4. Just as in the direct simulations, illustrated in Figure 25, the amplitude energy minima form a continuous locus as $r$ is varied. If we start with a configuration having circumferential wavenumbers in 
the regular Fibonacci sequence, this locus will pass through all other circumferential wavenumbers in the sequence. For example, if we were to plot the locus of minima corresponding to the choice of wavevectors shown in Fig. 26 between $r=11.8$ and $r=19.1$ in the $\left(\ell_{5},-\ell_{8}\right)$ plane, the energy minimizing locus would lie below the line $\ell_{5}+\ell_{8}=0$ and cover the whole range $(11.8,19.1)$. On the other hand, there is also another locus of energy minima which lies above the diagonal $\ell_{8}+\ell_{5}=0$ and starts at an $r$ value in between these two radii. On this branch, the $\ell_{13}$ has the opposite sign, the wavevector $\boldsymbol{k}_{13}$ lies to the right of the vertical line through $\ell=0$ and now the quadratic interaction $\boldsymbol{k}_{5}+\boldsymbol{k}_{13}$ draws the wavevector $\boldsymbol{k}_{18}$ towards the preferred wavenumber circle and the interaction between $\boldsymbol{k}_{8}$ and $\boldsymbol{k}_{13}$ pushes the resolvent $\boldsymbol{k}_{21}$ away. This gives rise to the beginning of a new continuous locus, $\boldsymbol{k}_{5}, \boldsymbol{k}_{13}, \boldsymbol{k}_{18}, \boldsymbol{k}_{31}$ and so on. Of course, in order to switch from one branch to another requires a large perturbation: one that takes the $\boldsymbol{k}_{13}$ mode from tilting left as it does in Fig. 26 to tilting right. The new set of solutions are marked by green on Fig. 36. In both Figures 35 and 36, the results are again overlaid on the Van Iterson diagram of Figure 6.

Result 5. By inspection, the set of amplitude equations for the nongradient case with the radial wavenumbers chosen to match those of the gradient case or the free energy $E$ of the gradient case are invariant under the self-similar transformations $A_{m}(r) \rightarrow A_{n}(r \varphi), \ell_{m}(r) \rightarrow-\ell_{n}(r \varphi), m \rightarrow n \approx m \varphi$. For large enough $m, n$ the last is valid to very good approximation.

Result 6 . What is the nature of the center manifold for patterns following Fibonacci progressions? We have discussed that, since the active mode set changes with radius, the graph of the amplitudes of the passive modes $P(A)$ expressed as a function of the amplitudes of the currently active modes $A$ changes with the generative annulus radius $r$. But the self-similarity property now gives us a way of defining a center manifold. Consider the center manifold in the interval $R_{m}, R_{n}$ including and between two hexagon configurations in which the active mode amplitudes are $A_{n-m}, A_{m}, A_{n}$ and $A_{m+n}$. It is the graph $P(m, n)=$ $P\left(A_{n-m}, A_{m}, A_{n}, A_{m+n}\right)$ expressing all other amplitudes as algebraic functions of the active four. The center manifold graph in the neighboring interval will be the same and gotten by simply shifting the amplitudes along the Fibonacci sequence. Therefore we can define the center manifold of the Fibonacci pattern to be that family of center manifolds $P(m, n)=P\left(A_{n-m}, A_{m}, A_{n}, A_{m+n}\right)$ obtained by mapping one $\left(R_{m}, R_{n}\right)$ interval to another. In the amplitude coordinate space, it will simply look like a rotation of the same surface.

\subsection{Transitions and whorls}

To this point, we have concentrated on Fibonacci progressions, showing both that they are associated with a locus of energy minima which is continuous in the generating radius parameter and that, at least in the disk geometry, they are the solution of the model which is realized when the generating radius is decreasing. In this section, we want to explore both transitions and the role of whorls. As we have already confessed, we do not at the moment have a definitive and complete understanding of all possible transitions and whether they are smooth or discontinuous, nor do we have a clear picture of the interplay and possible transition between whorl structures and Fibonacci spirals. We have instead rules of thumb which we now illustrate with a set of five case studies. Each study will provide an example of the transition types that are defined in detail and illustrated in the appendix. As described in Section 1.4, we characterize transitions by two indices: The first indicates if the transition is of Type I (discontinuous) or Type II (continuous). The second counts the number of wavevectors in the field $u$ that are preserved in the transition. In each case, the transition parameter corresponds to the radius $r$ of the generating annulus and transitions occur as this parameter is increased or decreased. Studies are carried out along a frustrum, joining two cylinders of different radii in order that the initial and final states can be seen to be stable.

1. We first illustrate a (II,2) transition, which we means that the transition is smooth and that the initial and final states share two wavevectors. The case study chosen and the result shown in Figure 37 addresses what happens when we decrease $r$ so that the hexagonal state with circumferential wavenumbers $(3,5,8)$, subsequently referred to as the $(3,5,8)$ state, makes a transition to the $(2,3,5)$ state. The taper is sufficiently fast so that instabilities described in case 3 below do not have time to manifest. We note the following: The transition is smooth in that the dominant wave vectors which are normal to the lines of constant phase change continuously. In particular, the transition reveals that the phase contours simply bend.

2. We now repeat the experiment in reverse order, namely we start with the $(2,3,5)$ state and, using the exact same taper, make the transition to the $(3,5,8)$ state. This is illustrated in Figure 38 . The 
transition is $(\mathrm{I}, 0)$ since the chirality of the pattern changes. It is not reversible. What happens is that in order for the five maximum phase contours to make the change to eight, dislocations which introduce new lines of maximum constant phase are created. Because of the appearance of dislocations to facilitate the transition, the transition is discontinuous although that conclusion, the fact that dislocations rather than bending phase contours are required to achieve the transition and that the Fibonacci progression is uninterrupted, might be viewed by colleagues of a practical bent as being somewhat semantic.

3. The rate of the taper matters. We now illustrate what happens when the time taken for the front to travel between two hexagonal states of a Fibonacci progression (see the discussion in Section 3.3) is both less than and greater than the growth time for potential instabilities. In Figure 37, we have shown the smooth $(\mathrm{II}, 2)$ transition between the $(3,5,8)$ and $(2,3,5)$ states when the taper is fast. In Figure 39, we show what happens when the taper is too slow. Additional modes with the circumferential wavenumbers 3 and 6 have been excited. This is an example of a (I,1) transition.

4. In the final two case studies, we set up the simulations differently in order to understand the behavior of the wavevectors before and after the transition. What we have done is to initialize a pattern, in this case a $(1,2,3)$ hexagonal pattern, on a cylinder with a radius just above its stability boundary. Since the growth of the instability is slow compared to the front speed, the $(1,2,3)$ pattern propagates along the cylinder for some time before the transition occurs. We can then isolate the location of the transition and study its properties. Figure 40a illustrates this $(1,2,3)$ pattern undergoing a transition to a $(2,2,4)$ whorl pattern through the insertion of one dislocation. This is a $(\mathrm{I}, 1)$ transition. In Figures $41 \mathrm{a}$ and $41 \mathrm{~b}$ we observe the wavevectors corresponding to the active modes of the pattern before and after the transition respectively. Notice that the wavevector with circumferential wavenumber 2 remains unchanged. Also note that the wavevector with circumferential wavenumber 5 is initially far inside the energetically preferred wavelength. Thus, this is similar to Eckhaus instabilities which occur in pattern-forming which still posess sign reversal symmetry.

5. Our final case study is an example of a $(\mathrm{I}, 0)$ transition from the whorl state $(g, g, 2 g)$ to $(g+1, g+$ $1,2 g+2)$. In Figure $40 \mathrm{~b}$, we have $g=2$, and the transition occurs through two dislocations in rapid succession. Figures $42 \mathrm{a}$ and $42 \mathrm{~b}$ illustrate the initial and final wavevectors along with the intermediate spiral state. The first dislocation takes the $(2,2,4)$ pattern to a $(2,3,5)$ pattern with the $m=5$ mode having radial (axial) wavenumber 0 . This pattern is strongly unstable for our isotropic model, so it undergoes another dislocation taking the pattern to a $(3,3,6)$ whorl planform.

\section{Conclusions}

The main goal of this review has been to make the case that mechanistic-based models informed by plants' biochemical and mechanical properties near their shoot apical meristems are consistent with, and richer than, models based on teleological approaches. But the story is far from over, and much remains to be done in particular in the experimental domain. In ongoing research, many of the of the modern pioneers (such as Meyerowitz, Kuhlemeier, Jönsson, and Dumais) are gaining more and more understanding of such behaviors as the interplay between local stress/strain fields and cell-to-cell auxin transport. Their results should inspire theoreticians to build even better models. At the same time, the theoretical results given in this review should inspire focused experiments. We have particularly in mind our predictions on the front propagation characteristics of the sunflower. The finding that there is variation in velocity between regions dominated by hexagons and those dominated by rhombi should be testable and measurable.

Another intriguing question is the universality of Fibonacci patterns. The model equation (23) and variants thereof can arise in many pattern-forming systems. There may be many contexts in which patterns are formed annulus-by-annulus leading to competition between the hexagonal planform preferred in planar geometries in systems with broken up-down symmetry (reflected in the quadratic term in 23) and rhombic and striped planforms.

We end this review with some questions which were stimulated by our studies but which may have broader ramifications.

1. Can we use pattern forming pde's to study optimal packing challenges? Why, at all, should there be a connection? What hidden properties does a pattern forming pde have which gives rise to optimal 
packing of its configuration of maxima? What other examples (e.g. the eye of the fruit fly [36]) might there be where this connection may be exploited?

2. It turns out that near the onset of the instability which gives rise to the pattern, the flow is effectively gradient. What connection, if any, does the effective free energy for the pde have with the energy minimizing functionals used in the teleological approaches?

3. How universal are Fibonacci patterns?

4. How can one tell a priori if a given pde will have solutions which are dominated by structures with self similar properties?

5. The teleological approach is based on the premise that, however a plant might achieve the outcome, the positioning of the phylla are such that each might enjoy maximum access to light and nutrients. But, one might also argue for another possibility: Sex and beauty! Just as our human eye is attracted to the pleasing architecture of flowers on a meristem, so too might the eyes of those insects which pollenate plants be attracted to this architecture because the pattern textures they see resonate with those natural patterns which arise because of the architecture of their brains. We know, for example, that the human brain stores a library of favored patterns. Experiments on folk undergoing hallucinogenic experiences confirm this. Why shouldn't the architecture of plants which have survived evolutionary battles be chosen so as to attract pollenators?

6. In what other contexts may evolving pattern fronts and Fibonacci sequences be important? Examples may include fruit fly eyes [36, generation of cryptographically strong codes [38, objective structures in materials [26, and the design of solar panels [4].

\section{Acknowledgements}

Many thanks to Elliot Meyerowitz for providing helpful feedback. This work was supported by NSF grant DMS-1308862 and Air Force grant FA9550-10-1-0064 to Alan C. Newell and NSF grant DMS-1022635 to Patrick D. Shipman.

[1] I. Adler, D. Barabé, and R. V. Jean. A history of the study of phyllotaxis. Annals of Botany, 80:231-244, 1997.

[2] P. Atela, C. Golé, and S. Hotton. A dynamical system for plant pattern formation: A rigorous analysis. J. Nonlinear Sci., 12:641-676, 2002.

[3] E. Benková, M. Michniewicz, M. Sauer, T. Teichmann, et al. Local, efflux-dependent auxin gradients as a common module for plant organ formation. Cell, 115:991-602, 2003.

[4] D.W. Boeringer. Phased array including a logarithmic spiral lattice of uniformly spaced radiating and receiving elements, August 13 2002. US Patent 6,433,754.

[5] A. H. Church. On the Relation of Phyllotaxis to Mechanical Laws. Williams and Norgate, 1904.

[6] D. J. Cosgrove. Growth of the plant cell wall. Nat. Rev. Mol. Cell Biol., 6:850-861, 2005.

[7] P. Barbier de Reuille, I. Bohn-Courseau, K. Ljung, H. Morin, N. Carraro, C. Godin, and J. Traas. Computer simulations reveal properties of the cell-cell signaling network at the shoot apex in arabidopsis. Proc. Nat. Acad. Sci., 103:1627-1632, 2006.

[8] S. Douady. The selection of phyllotactic patterns. In R. V. Jean and D. Barabé, editors, Symmetry in Plants, chapter 14, pages 335-358. World Scientific, 1998.

[9] S. Douady and Y. Couder. Phyllotaxis as a dynamical self-organizing process, part i: The spiral modes resulting from time-periodic iterations. J. Theor. Biol., 178:255-274, 1996.

[10] S. Douady and Y. Couder. Phyllotaxis as a dynamical self-organizing process, part ii: The spontaneous formation of a periodicity and the coexistance of spiral and whorled patterns. J. Theor. Biol., 178:295312, 1996. 
[11] S. Douady and Y. Couder. Phyllotaxis as a dynamical self-organizing process, part iii: The simulation of the transient regimes of ontogeny. J. Theor. Biol., 178:295-312, 1996.

[12] J. Dumais. Can mechanics control pattern formation in plants? Current Opinion in Plant Biology, 10:58-62, 2007.

[13] J. Dumais and D. Kwiatkowska. Analysis of surface growth in shoot apices. The Plant Journal, 31:229$241,2001$.

[14] D. Furihata and T. Matsuo. Discrete Variational Derivative Method: A Structure-Preserving Numerical Method for Partial Differential Equations. CRC Press, 2011.

[15] C. R. Goodall and P. B. Green. Quantitiative analysis of surface growth. Botanical Gazette, 147:1-15, 1986.

[16] P. B. Green. Expression of pattern in plants: Combining molecular and calculus-based biophysical paradigms. Am. J. Botany, 86:1059-1076, 1999.

[17] P. B. Green, C. R. Steele, and S. C. Rennich. Phyllotactic patterns: a biophysical mechanism for their origin. Ann. Bot., 77:515-528, 1996.

[18] O. Hamant, M. G. Heisler, H. Jönsson, et al. Developmental patterning by mechanical signals in Arabidopsis. Science, 322:1650-1655, 2008.

[19] M. G. Heisler, O. Hamant, P. Krupinski, M. Uttewaal, C. Ohno, H. Jönsson, J. Traas, and E. M. Meyerowitz. Alignment between pin1 polarity and microtubule orientation in the shoot apical meristem reveals a tight coupling between morphogenesis and auxin transport. PLOS Biology, 2011.

[20] M. G. Heisler, C. Ohno, P. Das, P. Sieber, et al. Patterns of auxin transport and gene expression during primoridum development revealed by live imaging of the Arabidopsis inflorescence meristerm. Current Biol., 15:1899-1911, 2005.

[21] L. Hernández and P. B. Green. Transductions for the expression of structural pattern: analysis in sunflower. The Plant Cell, 5:1725-1738, 1993.

[22] L. H. Hernández, A. Havelange, G. Bernier, and P. B. Green. Growth behavior of single epidermal cells during flower formation: sequential scanning electron micrographs provide kinematic patterns for Anagallis. Planta, 185:139-147, 1991.

[23] W. Hofmeister. Allegmeine morphologie der gewachse. In Handbuch der Physiologischen Botanik. Engelmann, 1868.

[24] S. Hotton. PhD thesis, University of California, Santa Cruz, 1999.

[25] S. Hotton, V. Johnson, J. Wilbarger, K. Zwieniecki, P. Atela, C. Golé, and J. Dumais. The possible and the actual in phyllotaxis: Bridging the gap between emperical observations and iterative models. J. Plant Growth Reg., 25:313-323, 2006.

[26] R. D. James. Objective structures. J. of the Mechanics and Physics of Solids, 54:2354-2390, 2006.

[27] R. V. Jean. Phyllotaxis: A Systematic Study in Plant Morphogenesis. Cambridge University Press, 1994.

[28] H. Jönsson, M. Heisler, B. E. Shapiro, E. M. Meyerowitz, and E. Mjolsness. An auxin-driven polarized transport model for phyllotaxis. Proc. Nat'l Acad. Sci., 103, 2006.

[29] M. Kuecken and A. C. Newell. Fingerprint formation. J. Theor. Biol., 235:71-83, 2005.

[30] D. K. Kwiatkowski. Ontogenetic changes of phyllotaxis in Anagallis Arvensis. L. Acta. Soc. Bot. Pol., 64:319-325, 1995. 
[31] D. K. Kwiatkowski and J. Dumais. Growth and morphogenesis at the vegetative shoot apex of Anagallis arvensis L. J. Exp. Bot., 54:1585-1595, 2003.

[32] L. S. Levitov. Energetic approach to phyllotaxis. Europhys. Lett., 14:533-539, 1991.

[33] L. S. Levitov. Phyllotaxis of flux lattices in layered superconductors. Phys. Rev. Lett., 66:224-227, 1991.

[34] D. J. B. Lloyd, B. Sandstede, D. Avitabile, and A. R. Champneys. Localized hexagon patterns of the planar swift-hohenberg equation. SIAM J. Appl. Dyn. Syst., 7:1049-1100, 2008.

[35] J. A. Lockhart. An analysis of irreversible plant cell elongation. J. Theor. Biol., 8:264-275, 1965.

[36] D. K. Lubensky, M. W. Pennington, B. I. Shraiman, and N. E. Baker. A dynamical model of ommatidial crystal formation. Proc. Nat'l Acad. Sci., 108:11145-11150, 2011.

[37] P. Martin and J. Lewis. Actin cables and epidermal movement in emybryonic wound healing. Nature, 360:179-183, 1992.

[38] F. C. Motta, P. D. Shipman, and B. D. Springer. Optimally topologically transitive orbits in discretetime dynamical systems. Submitted 2014.

[39] A. C. Newell and M. Pennybacker. Fibonacci patterns: Common or rare? Procedia IUTAM, 9:86-109, 2013.

[40] A. C. Newell and P. D. Shipman. Plants and Fibonacci. J. Stat. Phys., 121:5-6, 2005.

[41] A. C. Newell and P. D. Shipman. A new invariant in plant phyllotaxis. Analysis and Applications, 6:383-399, 2008.

[42] A. C. Newell, P. D. Shipman, and T. J. Cooke. Desert Plants Journal, 2013.

[43] A. C. Newell, P. D. Shipman, and Z. Sun. Phyllotaxis as an example of the symbiosis of mechanical forces and biochemical processes in living tissue. Plant Signal. Behav., 3:586-589, 2008.

[44] A. C. Newell, P. D. Shipman, and Z. Sun. Phyllotaxis: Cooperation and competition between mechanical and biochemical processes. J. Theor. Biol., 251:421-439, 2008.

[45] K. Okada, J. Ueda, M. K. Komaki, C. J. Bell, and Y. Shimura. Requirement of the auxin polar transport system in early stages of Arabidopsis floral bud formation. Plant Cell, 3:677-684, 1991.

[46] M. Pennybacker. PhD thesis, University of Arizona, 2013.

[47] D. Reinhardt, T. Mandel, and C. Kuhlemeier. Auxin regulates the initiation and radial position of plant lateral organs. Plant Cell, 12:507-518, 2000.

[48] D. Reinhardt, E.-R. Pesce, P. Stieger, T. Mandel, K. Baltensperger, M. Bennett, J. Traas, J. Friml, and C. Kuhlemeier. Regulation of phyllotaxis by polar auxin transport. Nature, 426:255-260, 2003.

[49] T. S. Sachs. Pattern formation in plant tissues. Cambridge University Press, 1991.

[50] S. Schwendener. Theorie der blattstellungen. Sitzungsber. Akad. Wiss. Berl., pages 741-773, 1909.

[51] P. D. Shipman. Discrete and continuous invariance in phyllotactic tilings. Phys. Rev. E, 81, 2010.

[52] P. D. Shipman and A. C. Newell. Phyllotactic patterns on plants. Phys. Rev. Lett., 92, 2004.

[53] P. D. Shipman and A. C. Newell. Polygonal planforms and phyllotaxis on plants. J. Theor. Biol., 236:154-197, 2005.

[54] R. S. Smith, S. Guyomarc'h, T. Mandel, D. Reinhardt, C. Kuhlemeier, and P. Prusinkiewicz. A plausible model for phyllotaxis. Proc. Nat'l Acad. Sci., 103:1301-1306, 2006.

[55] M. Snow and R. Snow. On the presence of tissue tension in apices. New Phytol., 50:484-485, 1951. 
[56] M. Snow and R. Snow. Minimum areas and leaf determination. Proc. R. Soc. B, 139:545-566, 1952.

[57] A. M. Turing. Morphogen theory of phyllotaxis. In P. T. Saunders, editor, Collected Works of A. M. Turing: Morphogenesis. North-Holland, 1952.

[58] G. van Iterson. Mathematische und mikroskopisch-anatomische Studien ueber Blattstellungen. Gustav Fischer, 1907.

[59] W. van Sarloos. Front propagation into unstable states. Phys. Rep., 386:29-222, 2003.

[60] R. F. Williams. The Shoot Apex and Leaf Growth. Cambridge University Press, 1975. 


\section{List of Figures}

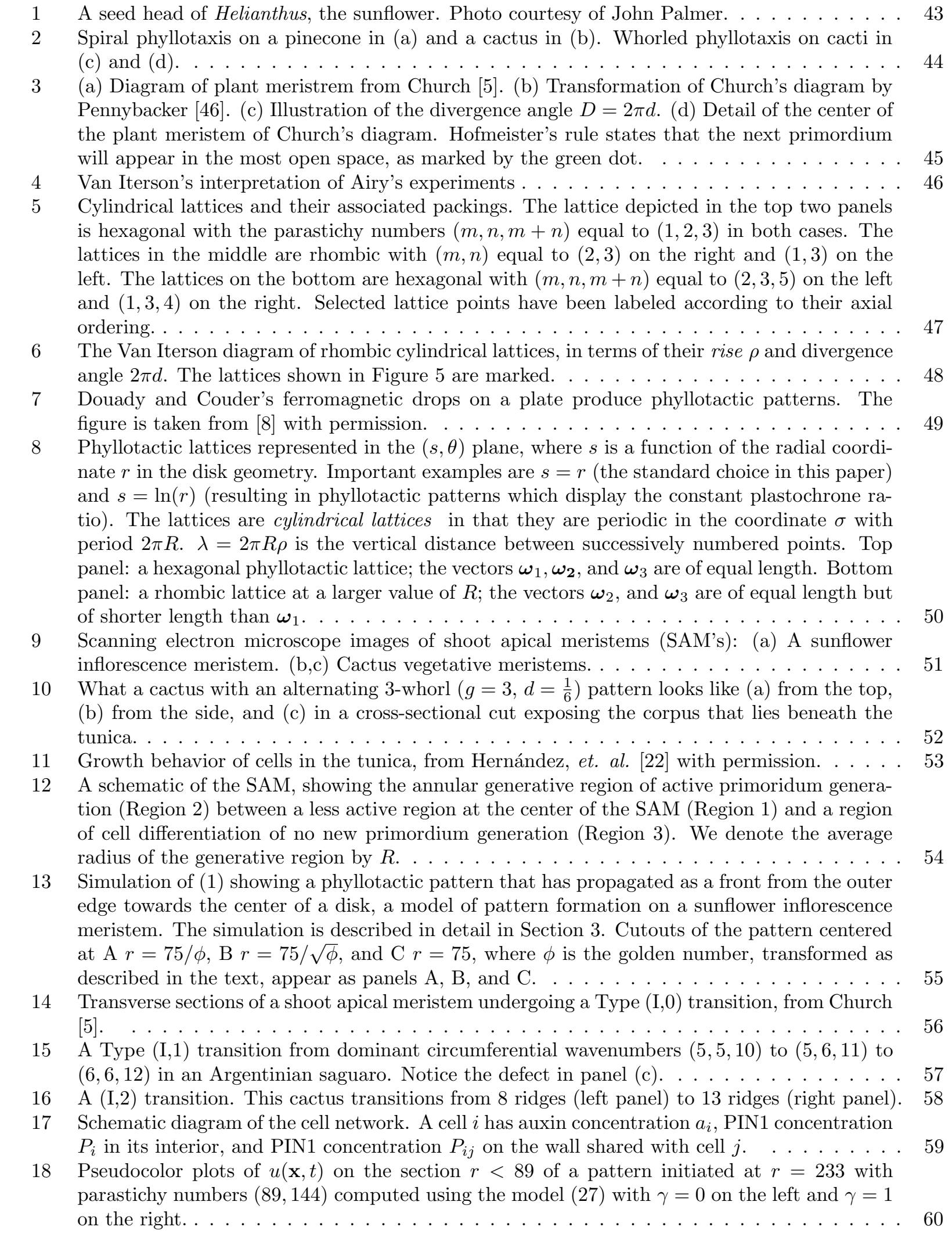


19 The invariant amplitude curve for amplitudes $a_{m_{j}}$ with $m_{j}$ from the regular Fibonacci sequence starting with $(1,2)$. The horizontal scaling emphasizes the self-similar property $a_{m_{j}}(r)=$ $a_{m_{j+1}}(\varphi r)$. From a solution of (27) with $\gamma=0$ on the left and $\gamma=1$ on the right. $\ldots \ldots .61$

20 Inside, the locus of wavevectors for modes with amplitude greater than $10 \%$ of the maximum value. Outside, the locus of wavevectors for irregular Fibonacci modes. The dashed semicircle indicates the preferred wavenumber $k_{0}$. From a solution of (27) with $\gamma=0$ on the left and $\gamma=1$ on the right.

21 A pseudocolor plot of $u(\mathbf{x}, t)$ on the section $r<89$ of a pattern initiated at $r=233$ with parastichy numbers $(89,144)$ computed using the nongradient model $(23)$.

22 The maximum values of the amplitude of all integer modes $8 \leq m \leq 89$, with a subset of circumferential wavenumbers indicated. The top figure corresponds to the $(1,2)$ sunflower and the bottom to the $(1,3)$ sunflower. $\ldots \ldots \ldots \ldots \ldots \ldots \ldots \ldots$

23 The invariant amplitude curve for amplitudes $a_{m_{j}}$ with $m_{j}$ from the Fibonacci-like sequences starting with $(1,2)$ and $(1,3)$. The horizontal scaling emphasizes the self-similar property $a_{m_{j}}(r)=a_{m_{j+1}}(\varphi r)$. .

24 The invariant curve in the $\left(\ell_{m}, \ell_{n}\right)$ wavevector space with $m$ and $n$ consecutive members of the appropriate Fibonacci-like sequence. The data have been reflected so that they all lie in the same quadrant. The shaded lines are the locus of rhombic lattices with the preferred wavelength along any single branch of the Van Iterson diagram. From a solution of (27) with $\gamma=0$ on the left and $\gamma=1$ on the right. $\ldots \ldots \ldots \ldots \ldots \ldots \ldots$

25 The rise $\rho$ and divergence angle $2 \pi d$ given by the local lattice structure at each radius on the $(1,2)$ sunflower, left, and $(1,3)$ sunflower, right. The shaded lines correspond exactly to Figure 6, with selected parastichy pairs indicated. Inset is detail of the data for small $\rho . \quad \ldots 66$

26 The wavevectors associated with active modes at four different radii. The upper-left figure illustrates a $(8,13,21)$ hexagonal pattern, and the lower-right figure illustrates the $(5,8,13)$ hexagonal pattern following the Fibonacci transition.

27 The front speed $\nu$ for the $(1,2)$ and $(1,3)$ sunflowers. Note that the horizontal axis has $\quad 67$

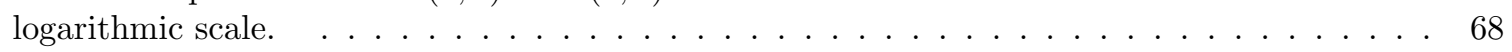

28 The local packing efficiency $\nu$ for the $(1,2)$ and $(1,3)$ sunflowers. Note that the horizontal axis has a logarithmic scale. $\ldots \ldots \ldots \ldots \ldots \ldots \ldots \ldots$

29 The local energy $\epsilon$ for the $(1,2)$ and $(1,3)$ sunflowers. Note that the horizontal axis has a

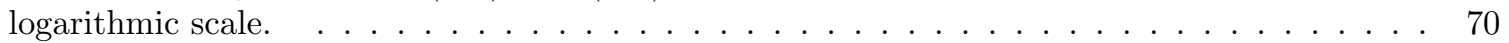

$30 \quad$ A contour plot of the average front speed of a $(1,2,3)$ hexagonal pattern on a cylinder which has its radius adjusted according to the nonlinear correction of the energetically preferred wavenumber. On the right half of the plot, it is clear that the front is pulled, since the speed does not depend significantly on $\beta$. On the left of the dashed line, the front is pushed, and on the right of the dashed line, the front is pulled. $\ldots \ldots \ldots \ldots \ldots \ldots$

31 The long-time dissipation rate on the annulus $34<r<55$. The initially large value is due to the propagation of the pattern-forming front into the annulus, and the later peaks are due to

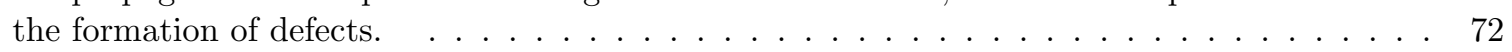

32 An invariant curve for the energy minimizing amplitudes $A_{m_{j}}$ with $\left\{m_{j}\right\}=\{1,2,3,5,8,13,21,34,55,89,144,233\}$. This has been computed by minimizing the energy over all amplitudes and wavenumbers on the locus of minima corresponding to the regular Fibonacci progression. The horizontal scaling emphasizes the self-similar property $A_{m_{j}}(r)=A_{m_{j+1}}(\varphi r)$.

33 An invariant curve for the energy minimizing wavenumbers $\ell_{m_{j}}$ with $\left\{m_{j}\right\}=\{1,2,3,5,8,13,21,34,5$ 吊, 89, 144,233$\}$. This has been computed by minimizing the energy over all amplitudes and wavenumbers on the locus of minima corresponding to the regular Fibonacci progression. The horizontal scaling emphasizes the self-similar property $\ell_{m_{j}}(r)=-\ell_{m_{j+1}}(\varphi r)$. . . . . . . . . . . . . . . 74

34 An invariant curve for the energy minimizing wavenumbers $\ell_{m_{j}}$ with $\left\{m_{j}\right\}=\{1,2,3,5,8,13,21,34,5 \$, 89,144,233\}$. This has been computed by minimizing the energy over all amplitudes and wavenumbers on the locus of minima corresponding to the regular Fibonacci progression. $\ldots \ldots \ldots \ldots$. . . 75

35 The divergence angle $2 \pi d$ and internodal spacing $\rho$ given by the wavevectors corresponding to the locus of energy minima of the amplitude equations along the main Fibonacci branches. Selected branches have been labeled with their parastichy numbers. $\ldots \ldots \ldots \ldots$ 
36 Again, the divergence angle $2 \pi d$ and internodal spacing $\rho$ given by the wavevectors corresponding to the locus of energy minima of the amplitude equations along the main Fibonacci branches. We also show an irregular Fibonacci branch, a new energy minimum corresponding to the selection of parastichy numbers $(5,13)$ rather than $(8,13)$ appearing at the end of the irregular locus. While $\rho$ decreases monotonically as $r$ increases on both branches, values of $r$ do not correspond to the same value of $\rho$ between branches. . . . . . . . . . . . . . . . . 77

37 The pattern laid down by a front propagating on a frustrum, which has been cut and unrolled, starting at $r \approx 7.5262$ and decreasing to $r \approx 4.6866$ along a distance of 110 space units. It has been initiated as a $(3,5,8)$ hexagonal pattern and ends as a $(2,3,5)$ hexagonal pattern. This is an example of a type (II,2) transition. $\quad$. . . . . . . . . . . . . . . . . . . . . . 78

38 The pattern laid down by a front propagating on a frustrum, which has been cut and unrolled, starting at $r \approx 4.6866$ and increasing to $r \approx 7.5262$ along a distance of 110 space units. It has been initiated as a $(2,3,5)$ hexagonal pattern and ends as a $(3,5,8)$ hexagonal pattern, although it shares none of its wavevectors with the initial pattern. This is an example of a type $(\mathrm{I}, 0)$ transition. $\quad \ldots \ldots \ldots \ldots \ldots \ldots$

39 The pattern laid down by a front propagating on a frustrum, which has been cut and unrolled, starting at $r \approx 7.5262$ and decreasing to $r \approx 4.6866$ along a distance of 200 space units. It has been initiated as a $(3,5,8)$ hexagonal pattern and ends as a $(3,3,6)$ hexagonal pattern.

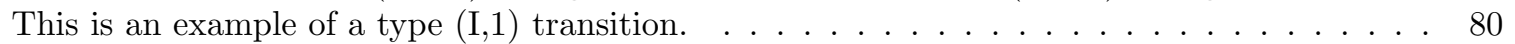

$40 \quad$ Illustrated here are two Type I, or discontinuous, transitions. . . . . . . . . . . . . . . . . . . 81

41 Shown above are the active wavevectors before and after the transition illustrated in Figure 40a $\quad$ There are two important features to note: (1) It is clear that $\boldsymbol{k}_{2}=\boldsymbol{k}_{2}^{-}$. The $m=2$ wavevector remains unchanged through the transition. (2) There is a four-mode resonance

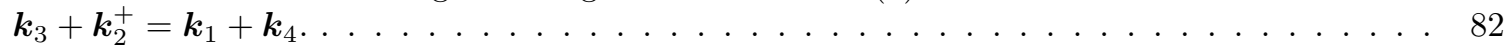

42 Shown above are the active wavevectors before and after each intermediate transition illustrated in Figure $40 \mathrm{~b}$. Overall, the transition begins with a $(2,2,4)$ whorl planform and ends with a $(3,3,6)$ whorl planform. $\ldots \ldots \ldots \ldots \ldots$ 


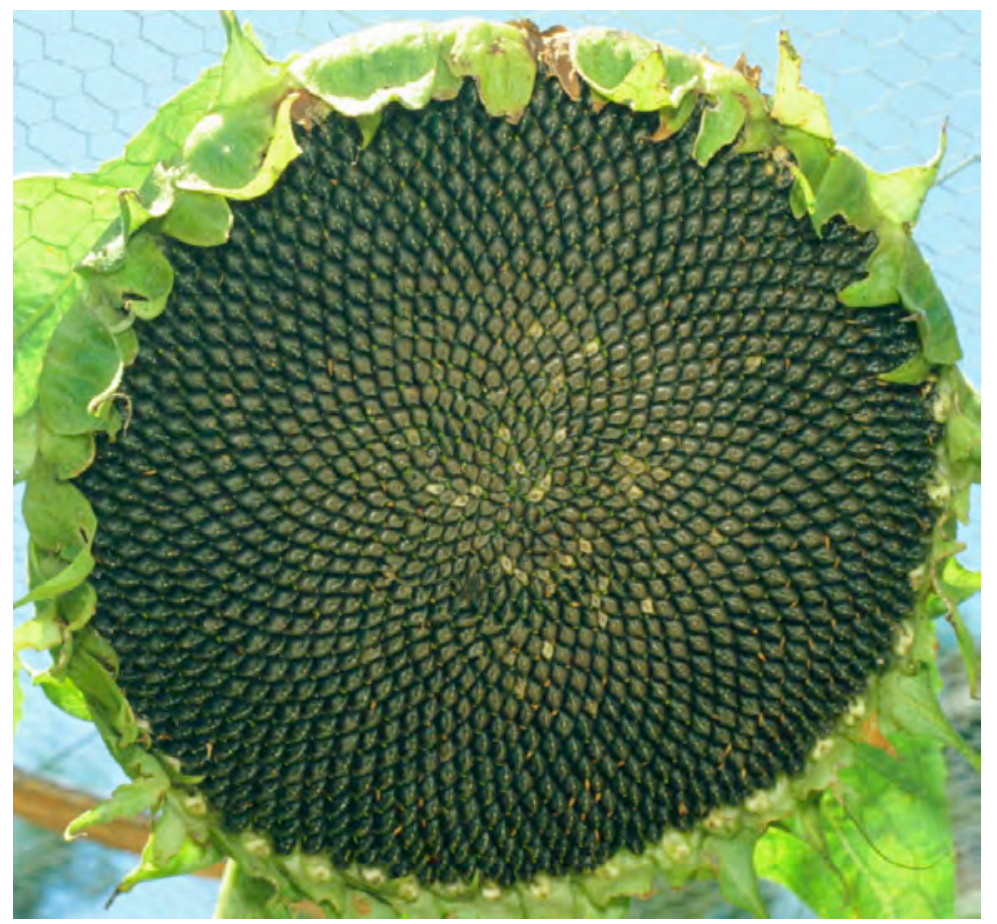

Figure 1: A seed head of Helianthus, the sunflower. Photo courtesy of John Palmer. 

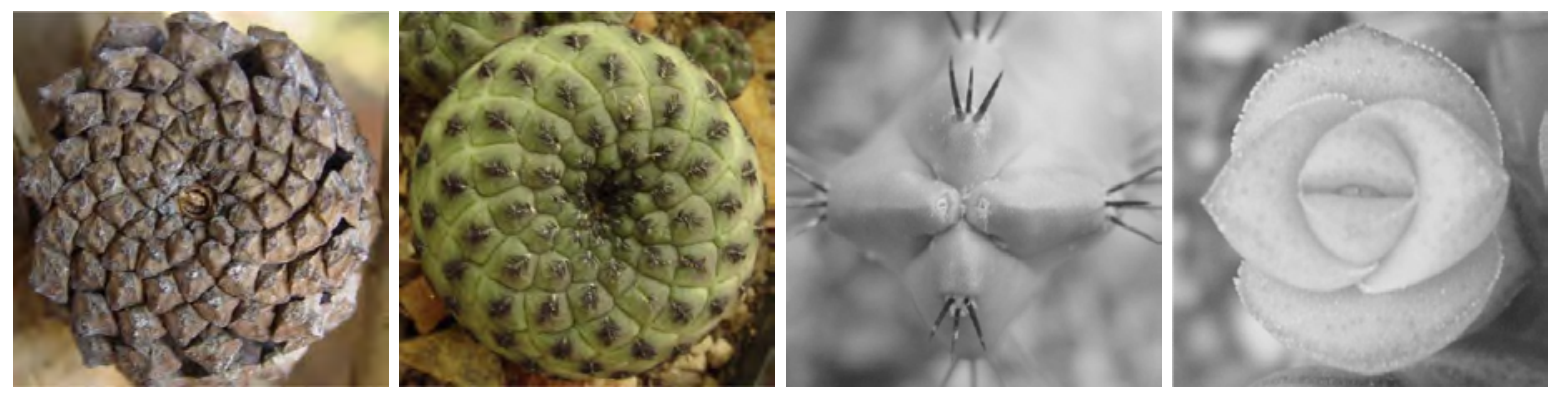

Figure 2: Spiral phyllotaxis on a pinecone in a and a cactus in b. Whorled phyllotaxis on cacti in (c) and d d . 

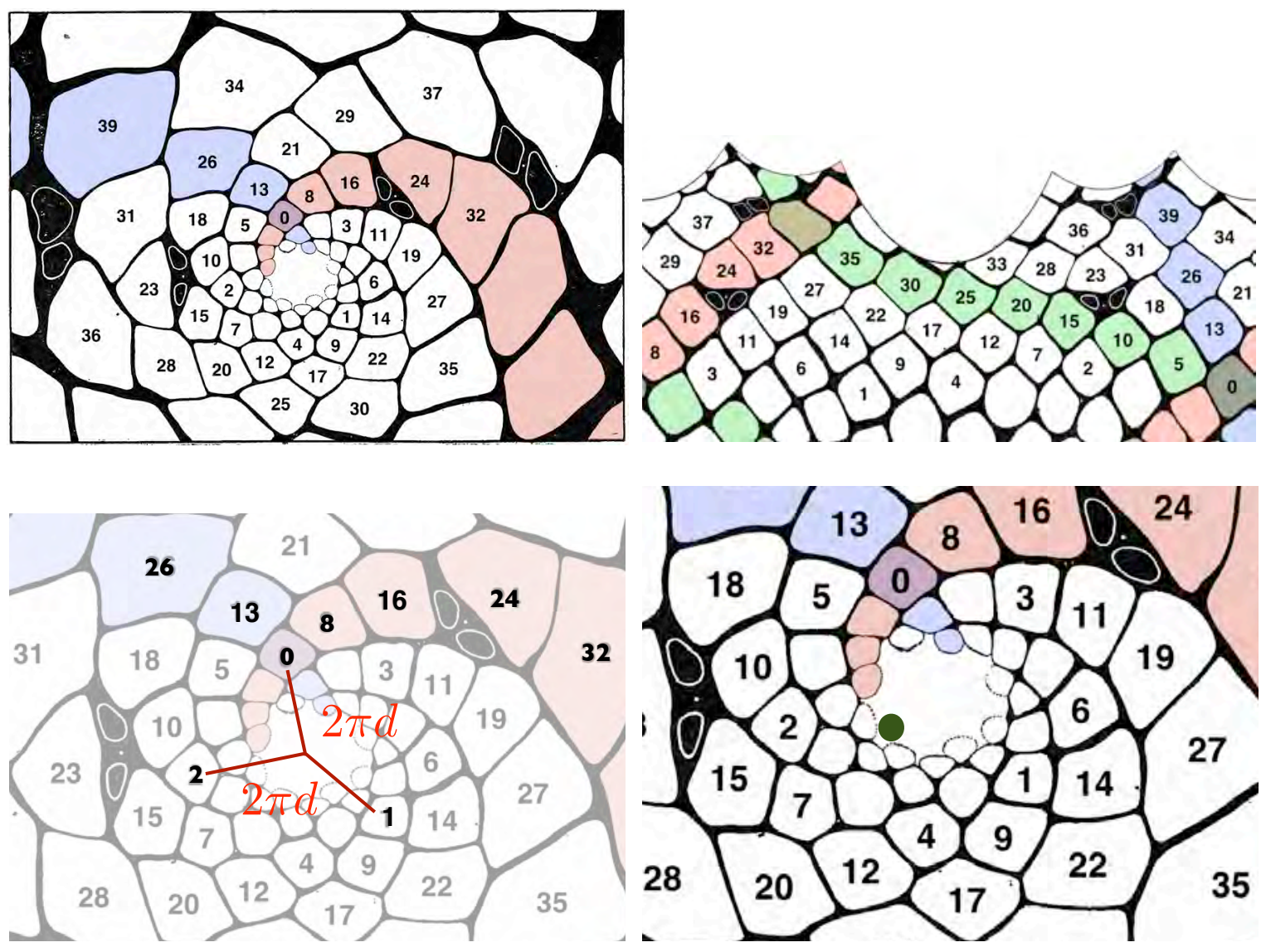

Figure 3: [a Diagram of plant meristrem from Church [5]. (b) Transformation of Church's diagram by Pennybacker 46]. [c] Illustration of the divergence angle $D=2 \pi d$. (d) Detail of the center of the plant meristem of Church's diagram. Hofmeister's rule states that the next primordium will appear in the most open space, as marked by the green dot. 


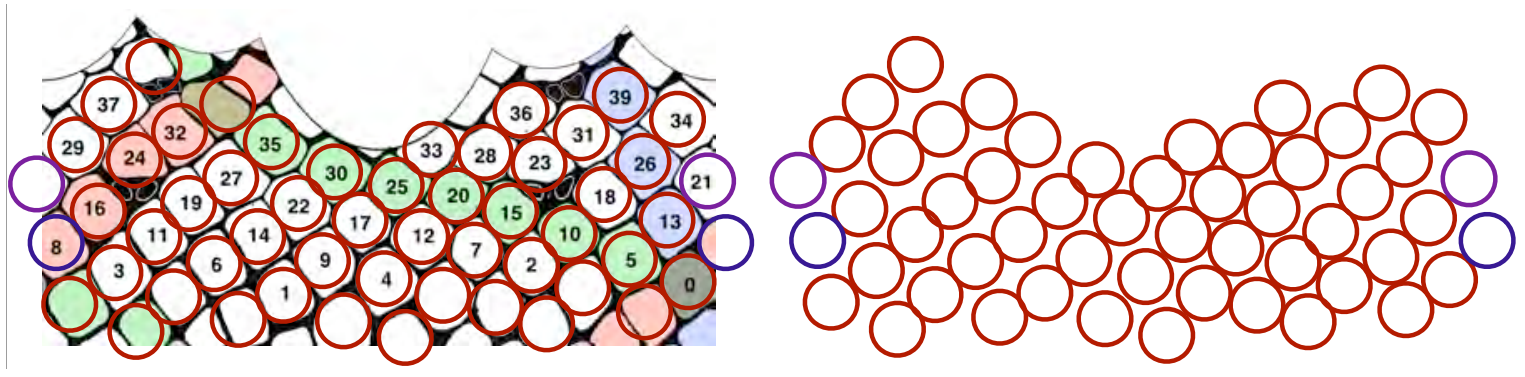

Figure 4: Van Iterson's interpretation of Airy's experiments 

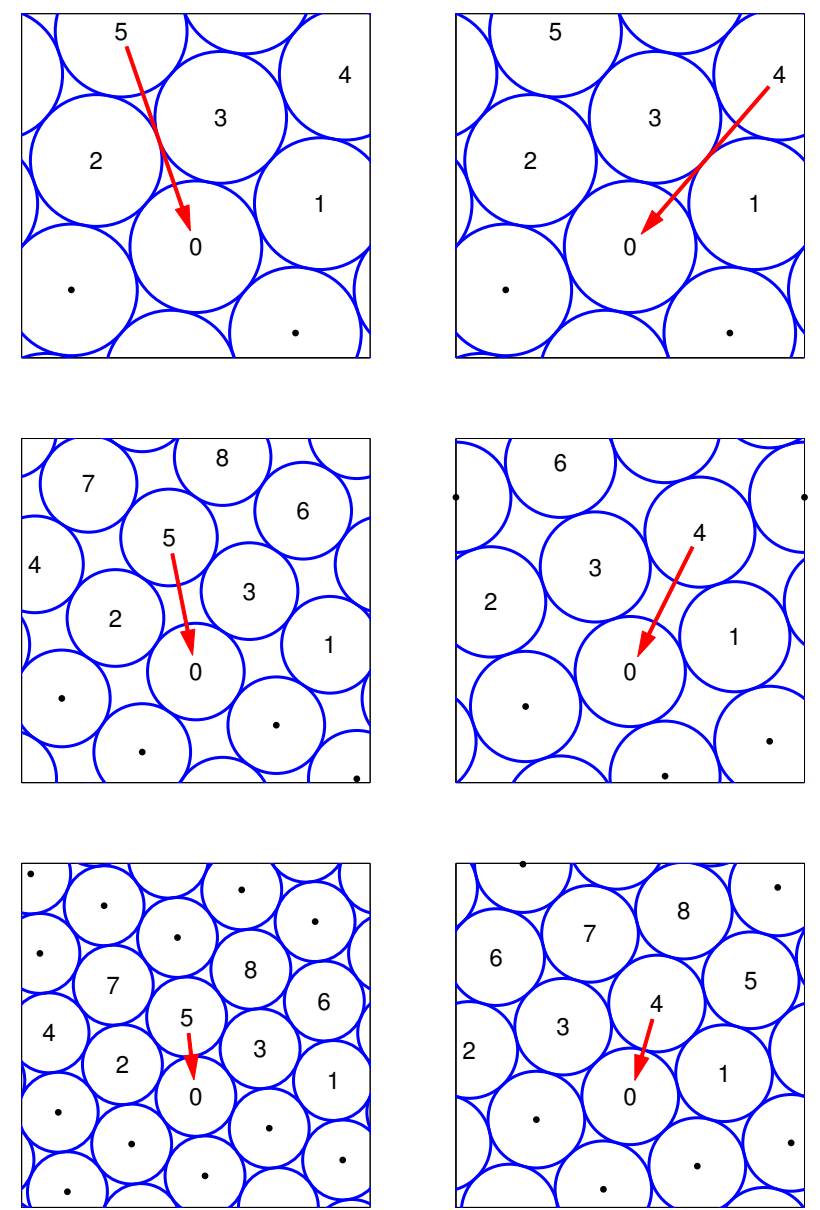

Figure 5: Cylindrical lattices and their associated packings. The lattice depicted in the top two panels is hexagonal with the parastichy numbers $(m, n, m+n)$ equal to $(1,2,3)$ in both cases. The lattices in the middle are rhombic with $(m, n)$ equal to $(2,3)$ on the right and $(1,3)$ on the left. The lattices on the bottom are hexagonal with $(m, n, m+n)$ equal to $(2,3,5)$ on the left and $(1,3,4)$ on the right. Selected lattice points have been labeled according to their axial ordering. 


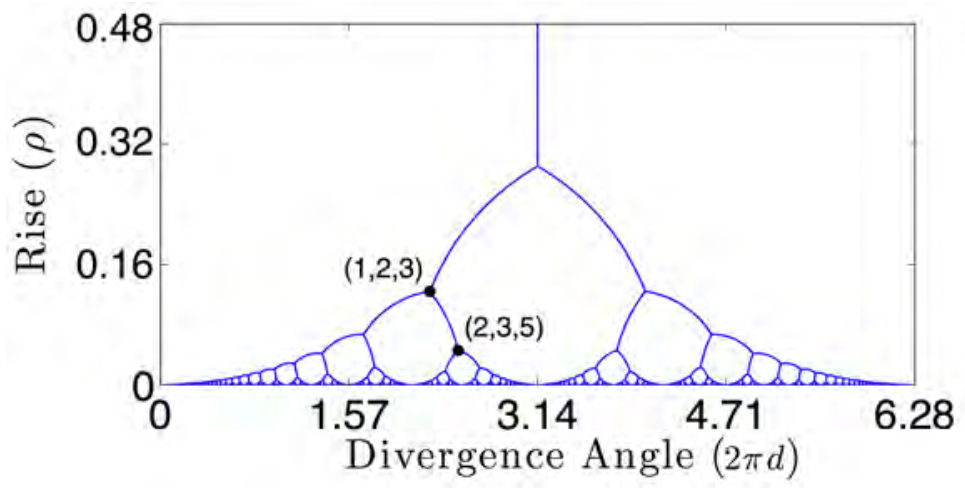

Figure 6: The Van Iterson diagram of rhombic cylindrical lattices, in terms of their rise $\rho$ and divergence angle $2 \pi d$. The lattices shown in Figure 5 are marked. 


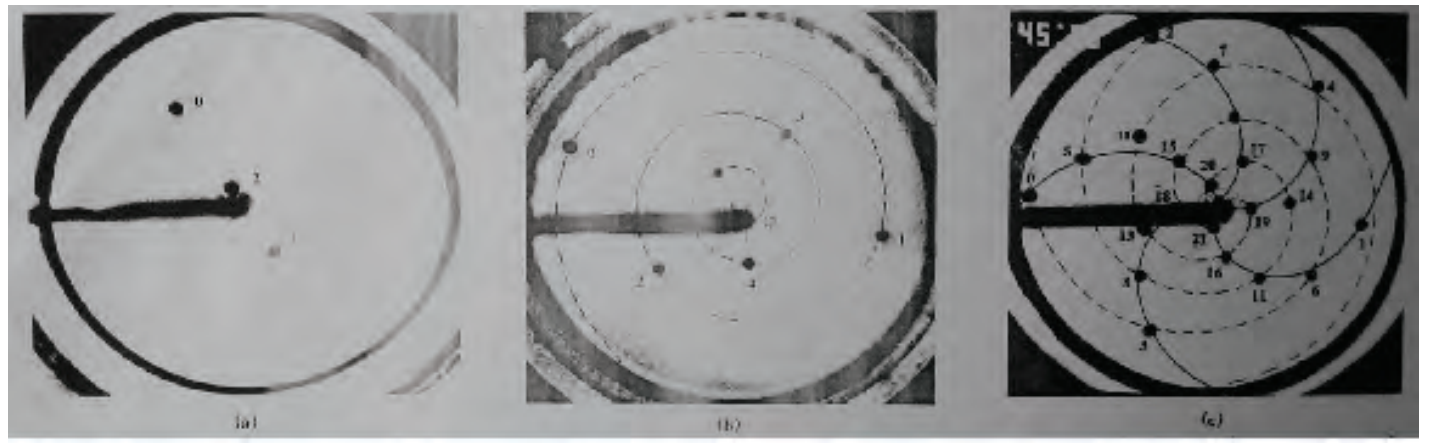

Figure 7: Douady and Couder's ferromagnetic drops on a plate produce phyllotactic patterns. The figure is taken from 8 with permission. 

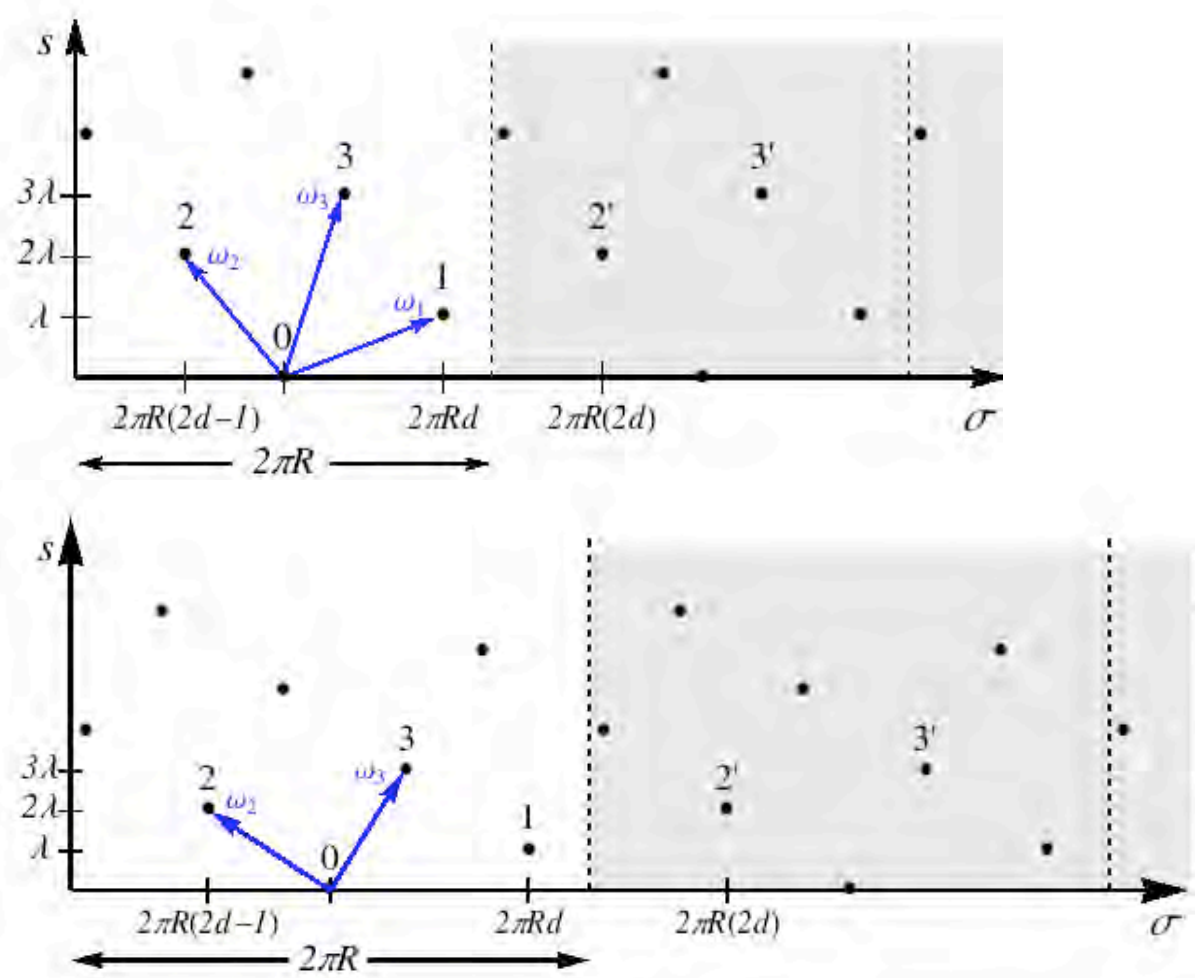

Figure 8: Phyllotactic lattices represented in the $(s, \theta)$ plane, where $s$ is a function of the radial coordinate $r$ in the disk geometry. Important examples are $s=r$ (the standard choice in this paper) and $s=\ln (r)$ (resulting in phyllotactic patterns which display the constant plastochrone ratio). The lattices are cylindrical lattices in that they are periodic in the coordinate $\sigma$ with period $2 \pi R . \lambda=2 \pi R \rho$ is the vertical distance between successively numbered points. Top panel: a hexagonal phyllotactic lattice; the vectors $\boldsymbol{\omega}_{1}, \boldsymbol{\omega}_{\mathbf{2}}$, and $\boldsymbol{\omega}_{3}$ are of equal length. Bottom panel: a rhombic lattice at a larger value of $R$; the vectors $\boldsymbol{\omega}_{2}$, and $\boldsymbol{\omega}_{3}$ are of equal length but of shorter length than $\boldsymbol{\omega}_{1}$ 

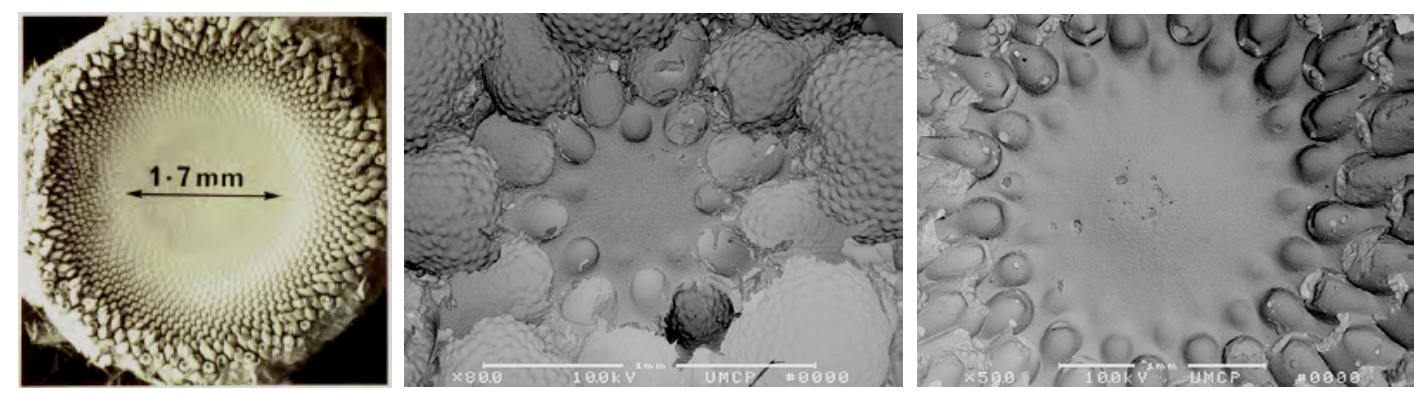

Figure 9: Scanning electron microscope images of shoot apical meristems (SAM's): (a) A sunflower inflorescence meristem. $(\mathrm{b}, \mathrm{c})$ Cactus vegetative meristems. 

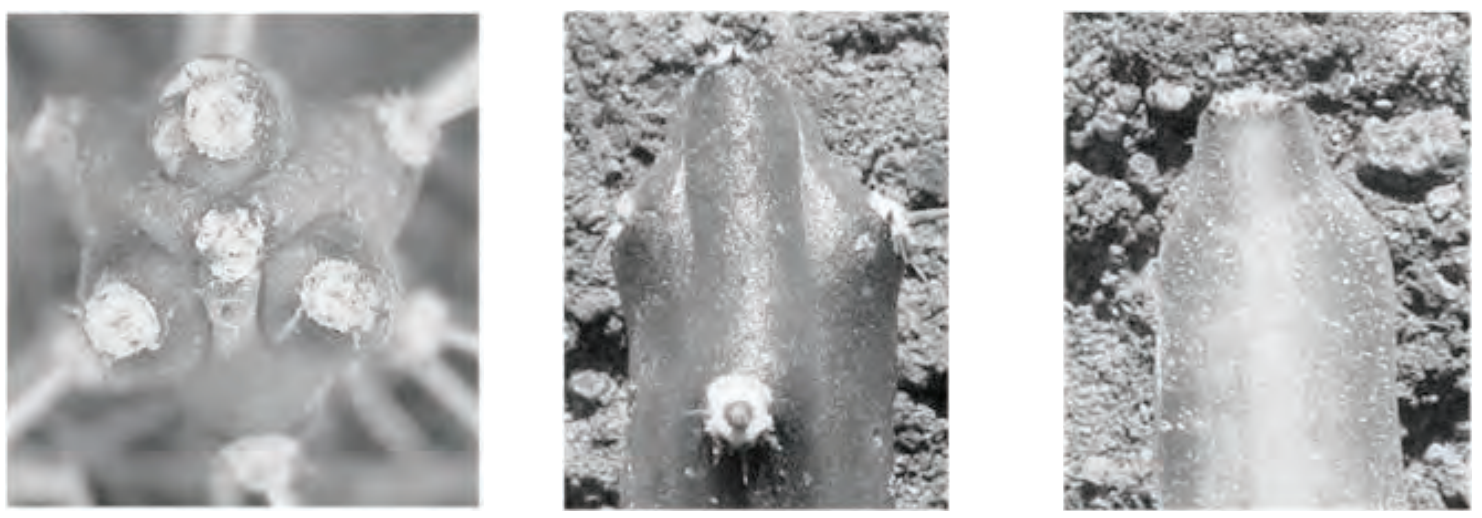

Figure 10: What a cactus with an alternating 3-whorl $\left(g=3, d=\frac{1}{6}\right)$ pattern looks like (a) from the top, b from the side, and (c) in a cross-sectional cut exposing the corpus that lies beneath the tunica. 

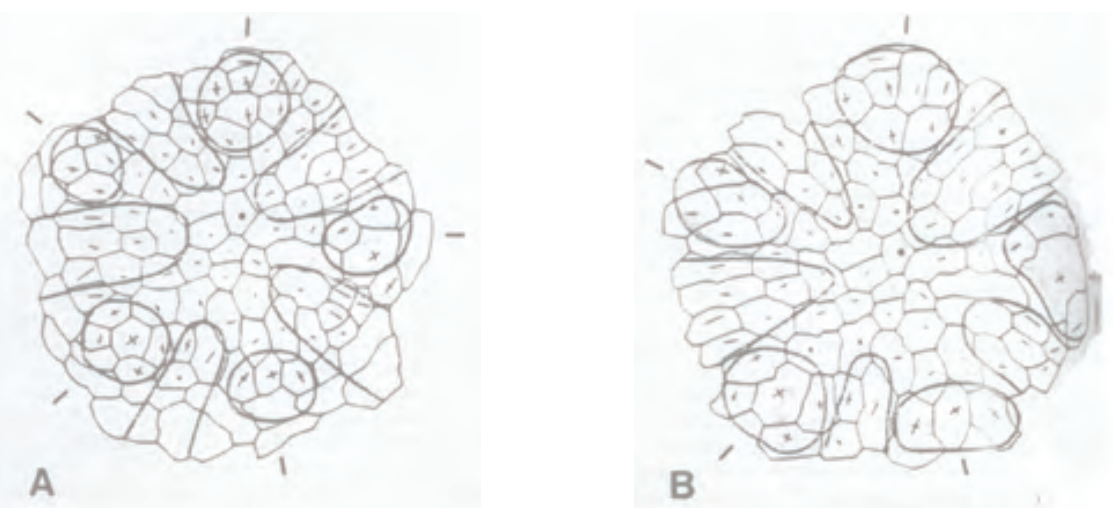

Figure 11: Growth behavior of cells in the tunica, from Hernández, et. al. 22] with permission. 


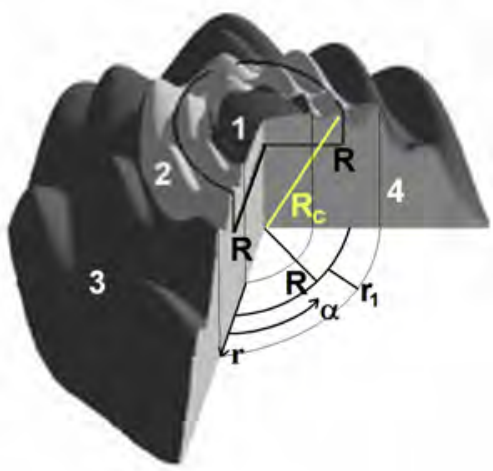

Figure 12: A schematic of the SAM, showing the annular generative region of active primoridum generation (Region 2) between a less active region at the center of the SAM (Region 1) and a region of cell differentiation of no new primordium generation (Region 3 ). We denote the average radius of the generative region by $R$. 

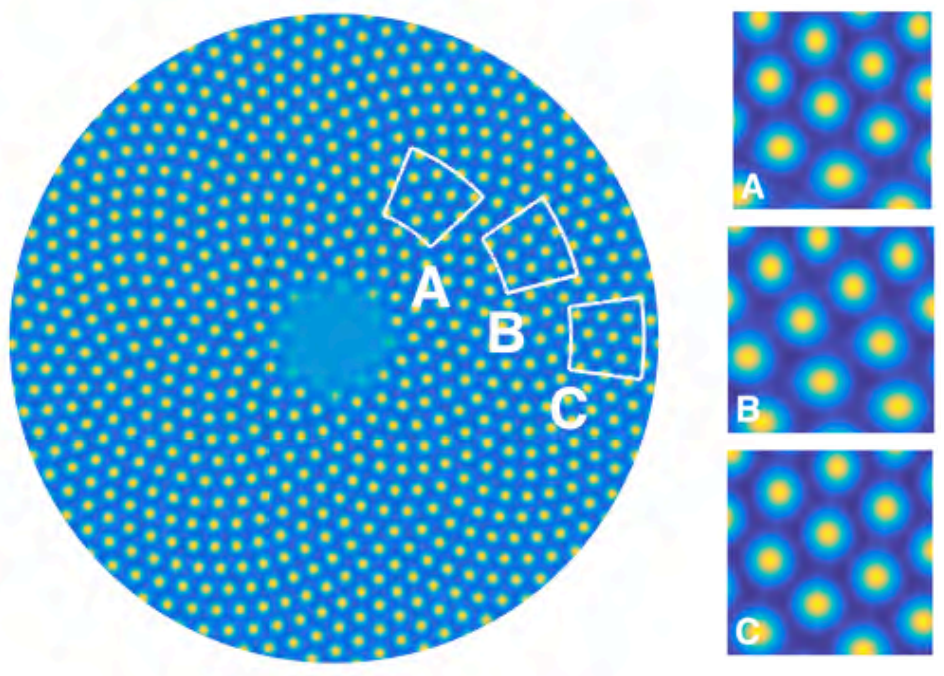

Figure 13: Simulation of 1 showing a phyllotactic pattern that has propagated as a front from the outer edge towards the center of a disk, a model of pattern formation on a sunflower inflorescence meristem. The simulation is described in detail in Section 3 Cutouts of the pattern centered at A $r=75 / \phi, \mathrm{B} r=75 / \sqrt{\phi}$, and $\mathrm{C} r=75$, where $\phi$ is the golden number, transformed as described in the text, appear as panels A, B, and C. 


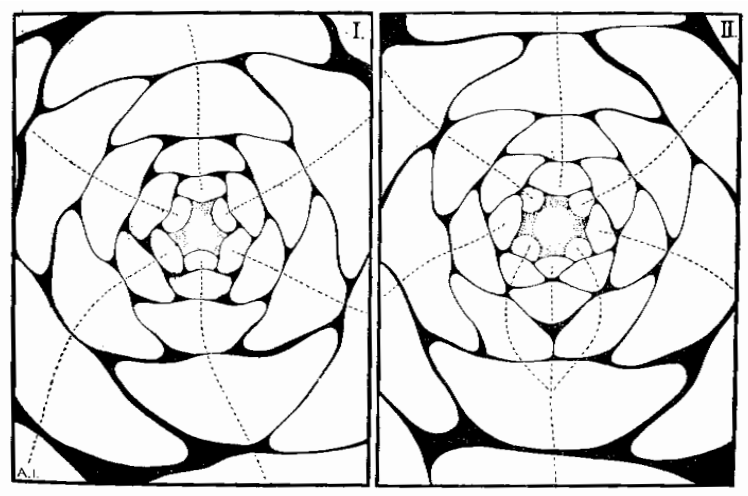

Figure 14: Transverse sections of a shoot apical meristem undergoing a Type $(\mathrm{I}, 0)$ transition, from Church [5]. 

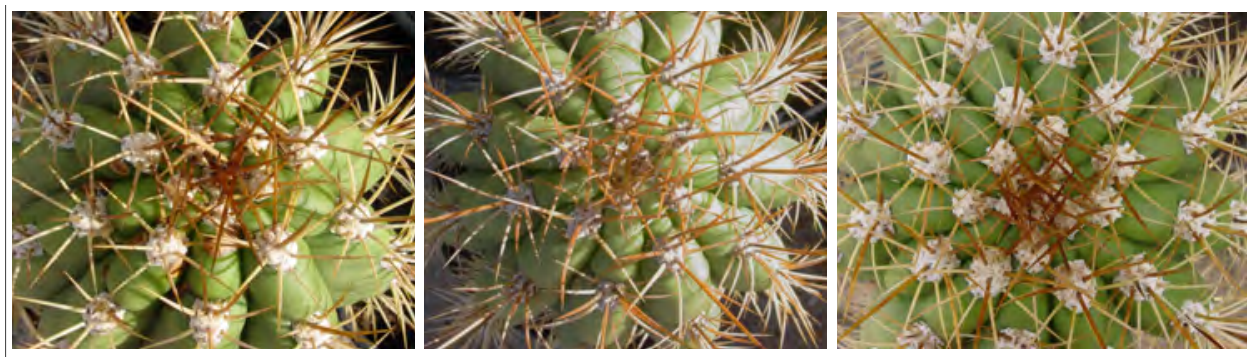

Figure 15: A Type (I,1) transition from dominant circumferential wavenumbers $(5,5,10)$ to $(5,6,11)$ to $(6,6,12)$ in an Argentinian saguaro. Notice the defect in panel (c). 

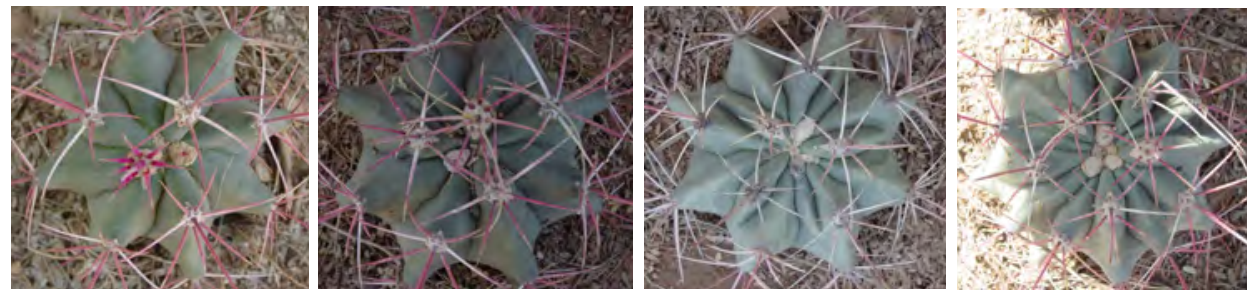

Figure 16: A (I,2) transition. This cactus transitions from 8 ridges (left panel) to 13 ridges (right panel). 


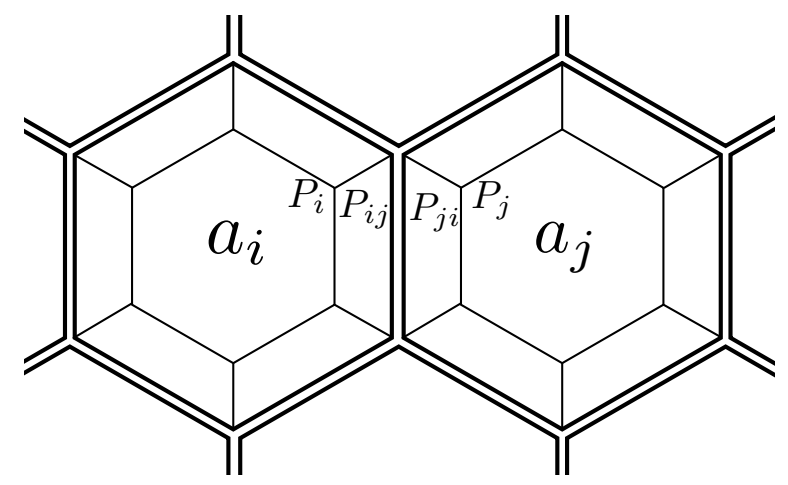

Figure 17: Schematic diagram of the cell network. A cell $i$ has auxin concentration $a_{i}$, PIN1 concentration $P_{i}$ in its interior, and PIN1 concentration $P_{i j}$ on the wall shared with cell $j$. 

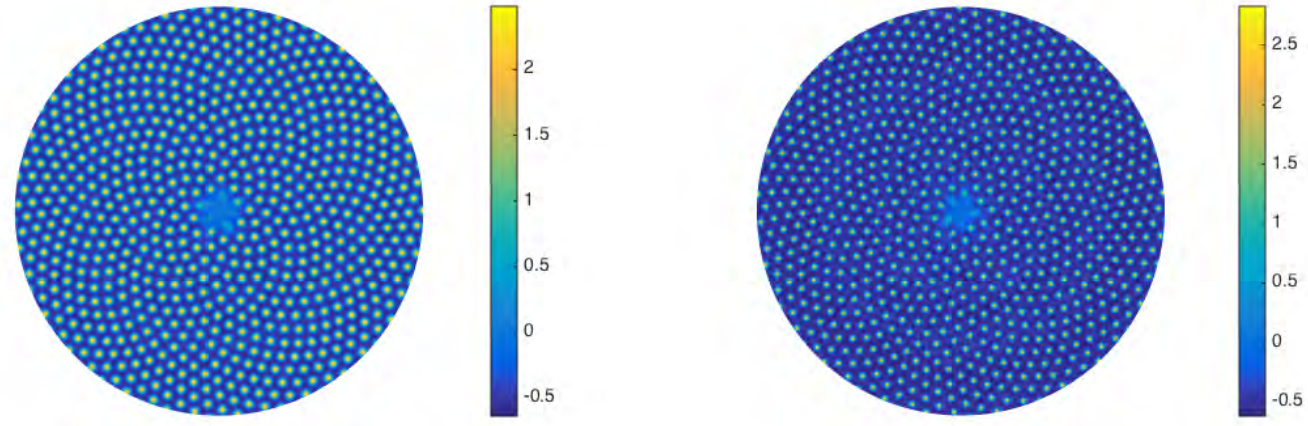

Figure 18: Pseudocolor plots of $u(\mathbf{x}, t)$ on the section $r<89$ of a pattern initiated at $r=233$ with parastichy numbers $(89,144)$ computed using the model 27] with $\gamma=0$ on the left and $\gamma=1$ on the right. 

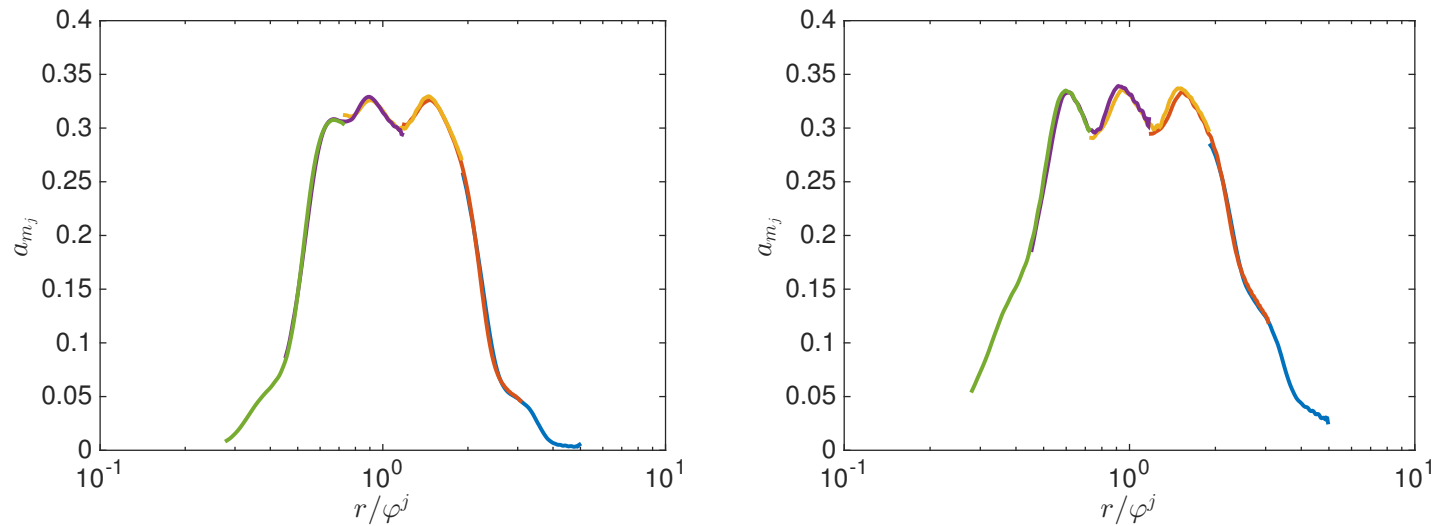

Figure 19: The invariant amplitude curve for amplitudes $a_{m_{j}}$ with $m_{j}$ from the regular Fibonacci sequence starting with (1,2). The horizontal scaling emphasizes the self-similar property $a_{m_{j}}(r)=a_{m_{j+1}}(\varphi r)$. From a solution of 27) with $\gamma=0$ on the left and $\gamma=1$ on the right. 

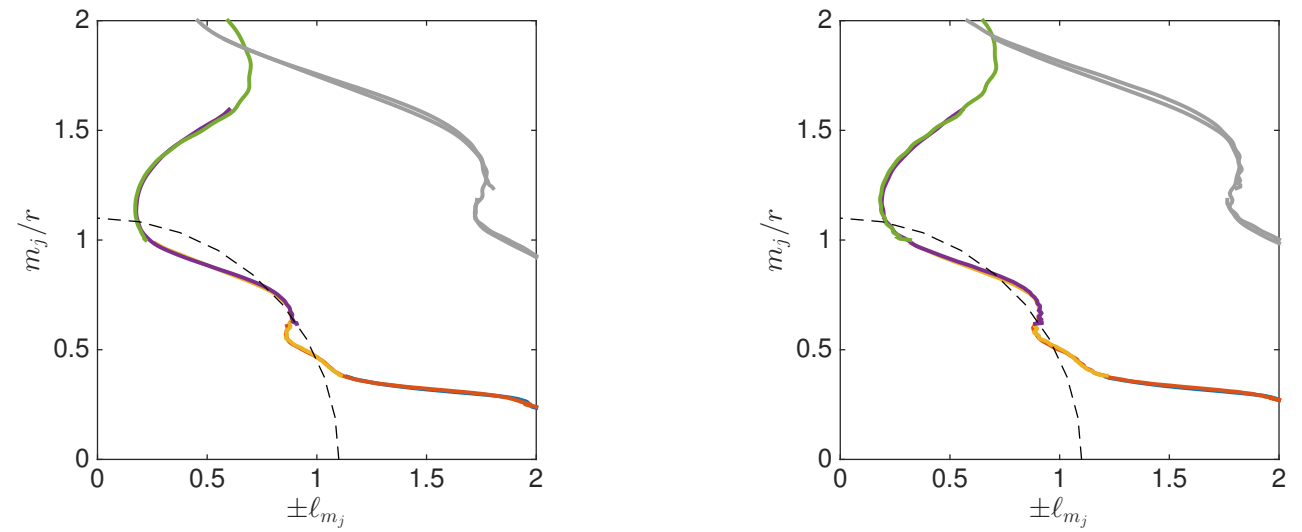

Figure 20: Inside, the locus of wavevectors for modes with amplitude greater than $10 \%$ of the maximum value. Outside, the locus of wavevectors for irregular Fibonacci modes. The dashed semicircle indicates the preferred wavenumber $k_{0}$. From a solution of 27] with $\gamma=0$ on the left and $\gamma=1$ on the right 


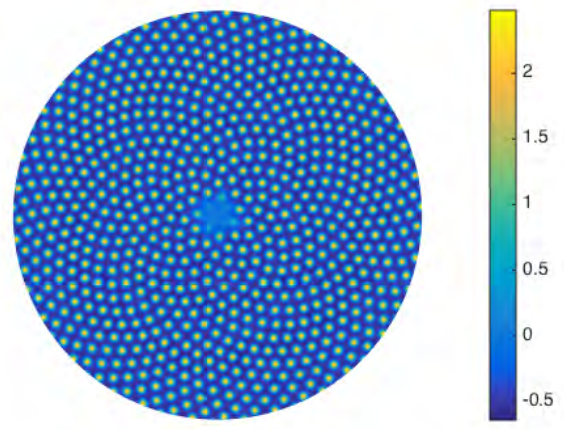

Figure 21: A pseudocolor plot of $u(\mathbf{x}, t)$ on the section $r<89$ of a pattern initiated at $r=233$ with parastichy numbers $(89,144)$ computed using the nongradient model 23 . 

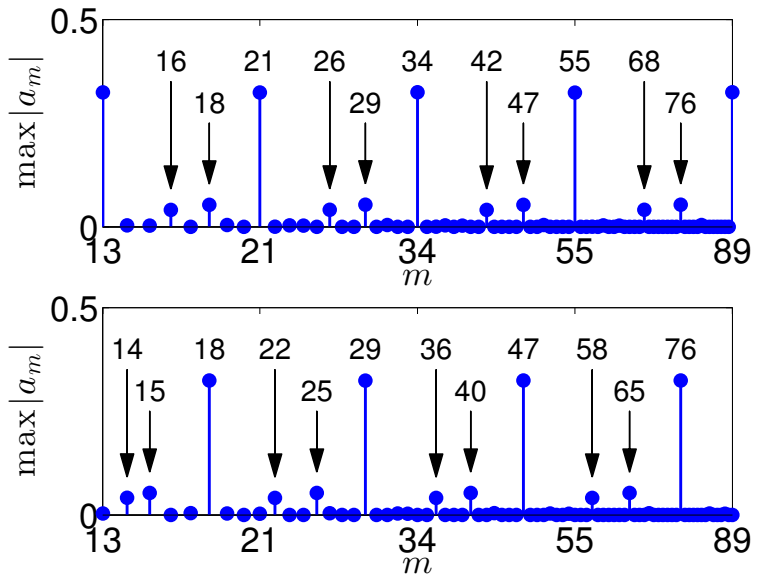

Figure 22: The maximum values of the amplitude of all integer modes $8 \leq m \leq 89$, with a subset of circumferential wavenumbers indicated. The top figure corresponds to the $(1,2)$ sunflower and the bottom to the $(1,3)$ sunflower.

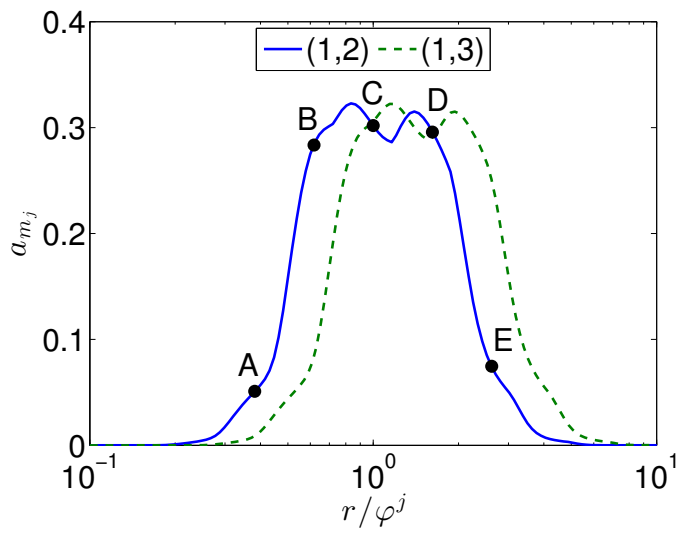

Figure 23: The invariant amplitude curve for amplitudes $a_{m_{j}}$ with $m_{j}$ from the Fibonacci-like sequences starting with $(1,2)$ and $(1,3)$. The horizontal scaling emphasizes the self-similar property $a_{m_{j}}(r)=a_{m_{j+1}}(\varphi r)$. 

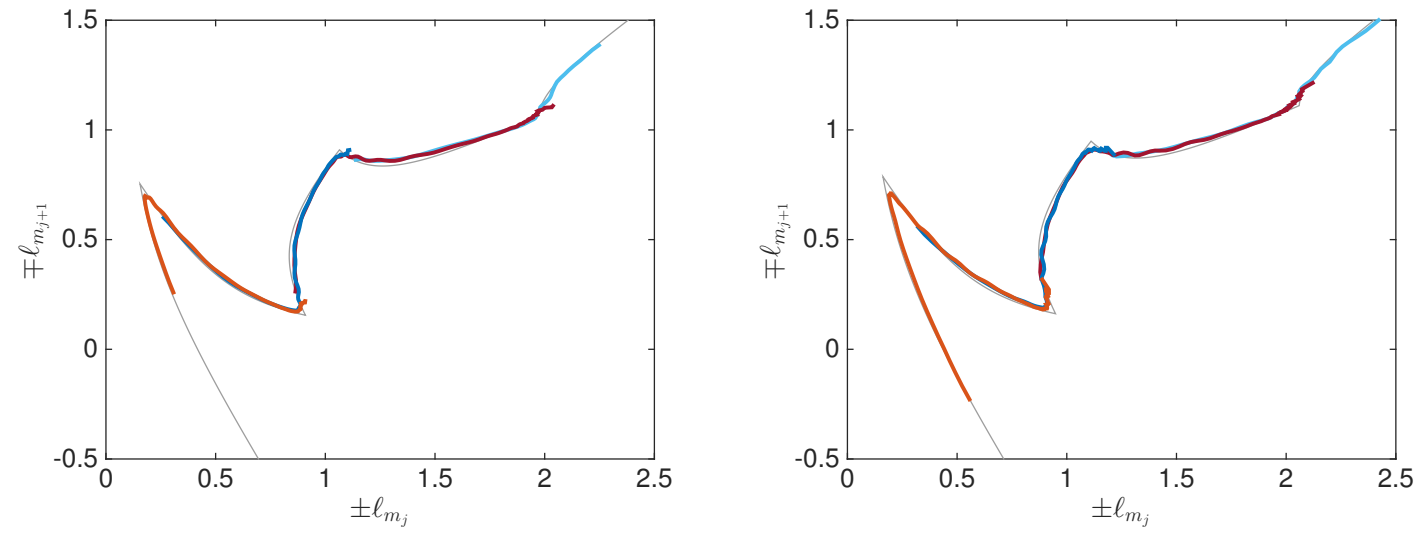

Figure 24: The invariant curve in the $\left(\ell_{m}, \ell_{n}\right)$ wavevector space with $m$ and $n$ consecutive members of the appropriate Fibonaccilike sequence. The data have been reflected so that they all lie in the same quadrant. The shaded lines are the locus of rhombic lattices with the preferred wavelength along any single branch of the Van Iterson diagram. From a solution of 27] with $\gamma=0$ on the left and $\gamma=1$ on the right. 

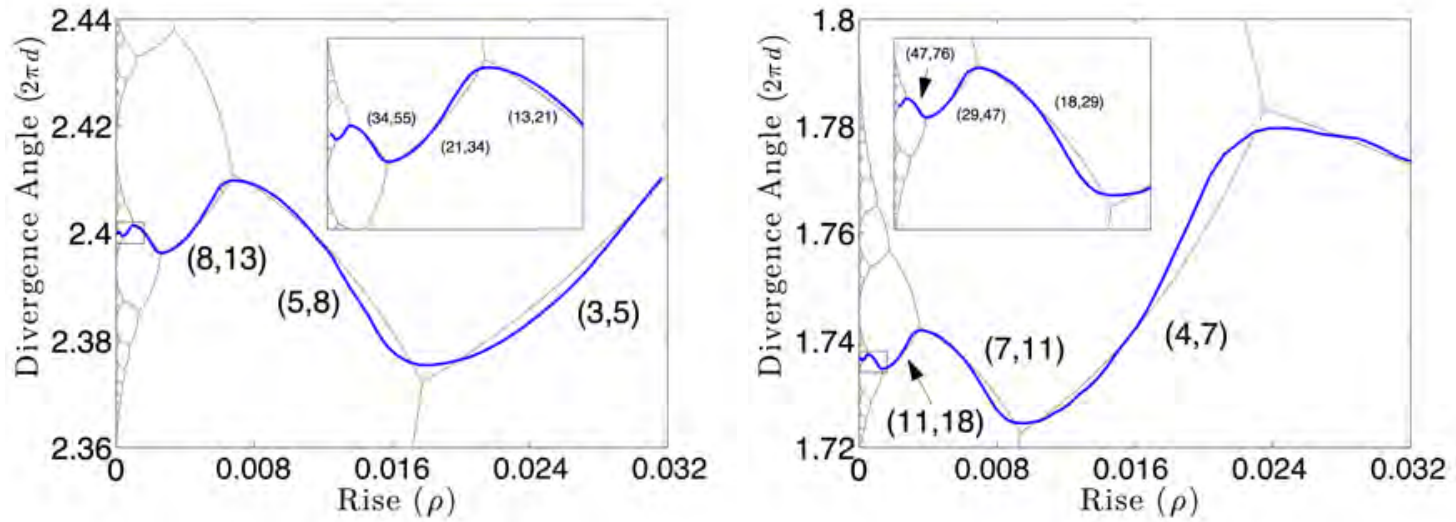

Figure 25: The rise $\rho$ and divergence angle $2 \pi d$ given by the local lattice structure at each radius on the $(1,2)$ sunflower, left, and $(1,3)$ sunflower, right. The shaded lines correspond exactly to Figure 6 , with selected parastichy pairs indicated. Inset is detail of the data for small $\rho$. 

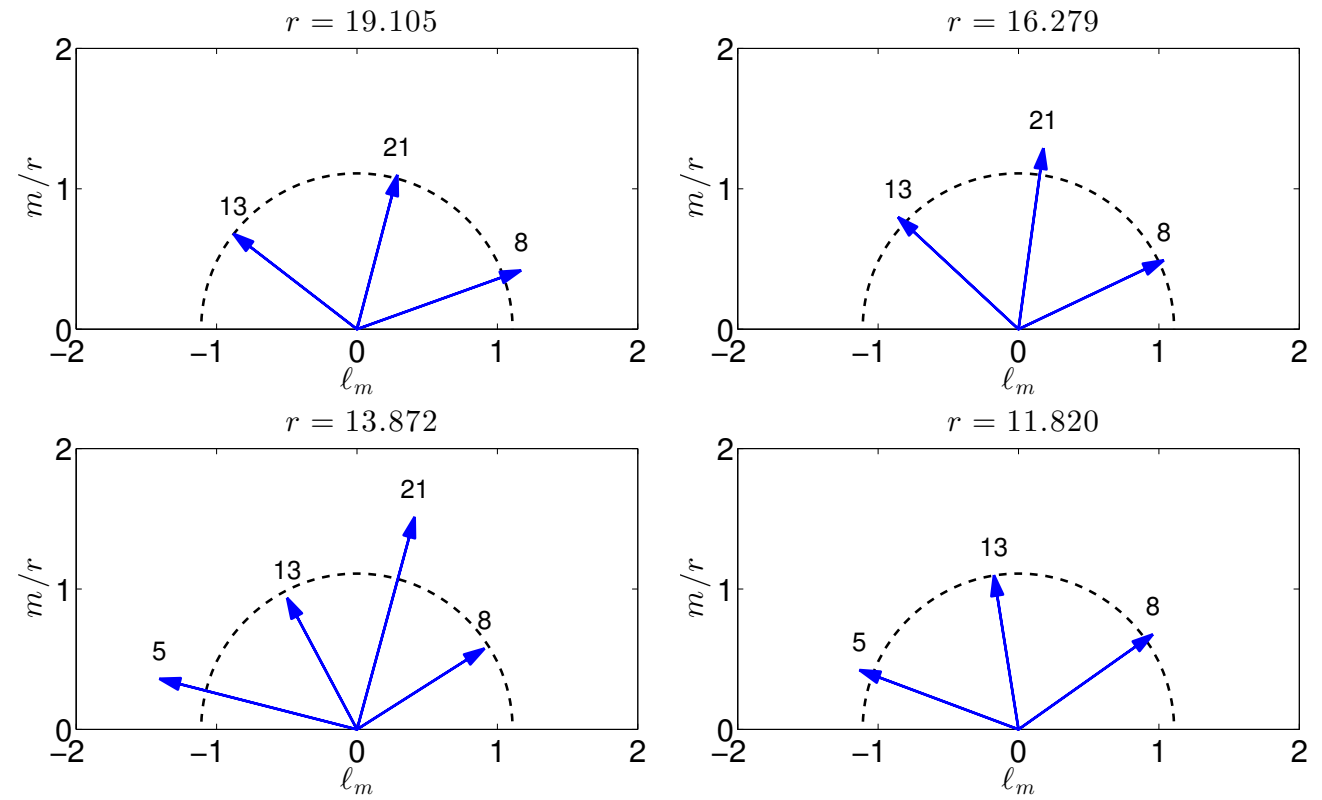

Figure 26: The wavevectors associated with active modes at four different radii. The upper-left figure illustrates a $(8,13,21)$ hexagonal pattern, and the lower-right figure illustrates the $(5,8,13)$ hexagonal pattern following the Fibonacci transition. 


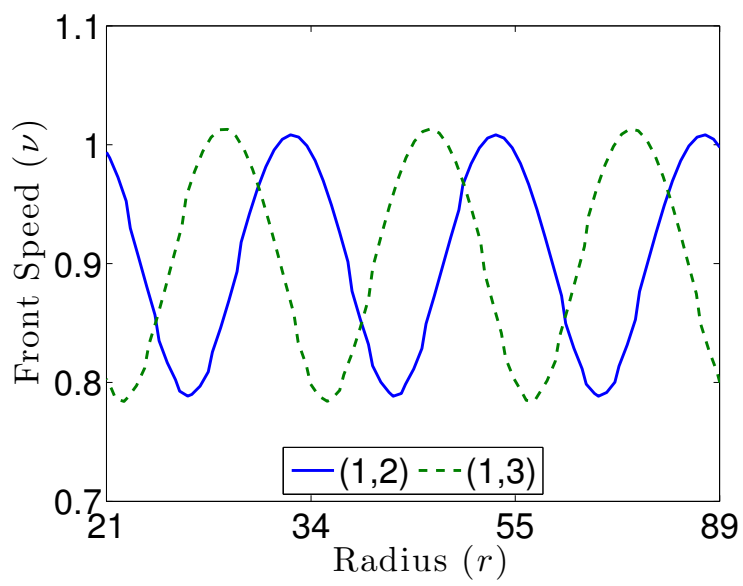

Figure 27: The front speed $\nu$ for the $(1,2)$ and $(1,3)$ sunflowers. Note that the horizontal axis has a logarithmic scale. 


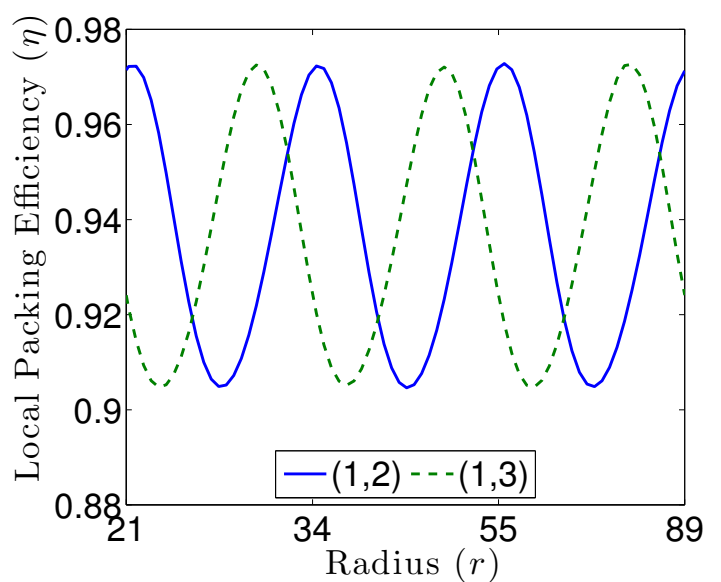

Figure 28: The local packing efficiency $\nu$ for the $(1,2)$ and $(1,3)$ sunflowers. Note that the horizontal axis has a logarithmic scale. 


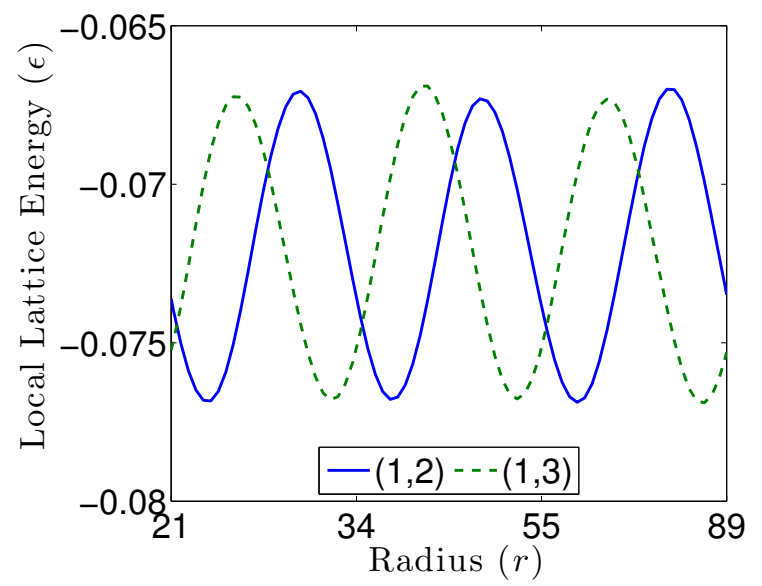

Figure 29: The local energy $\epsilon$ for the $(1,2)$ and $(1,3)$ sunflowers. Note that the horizontal axis has a logarithmic scale. 


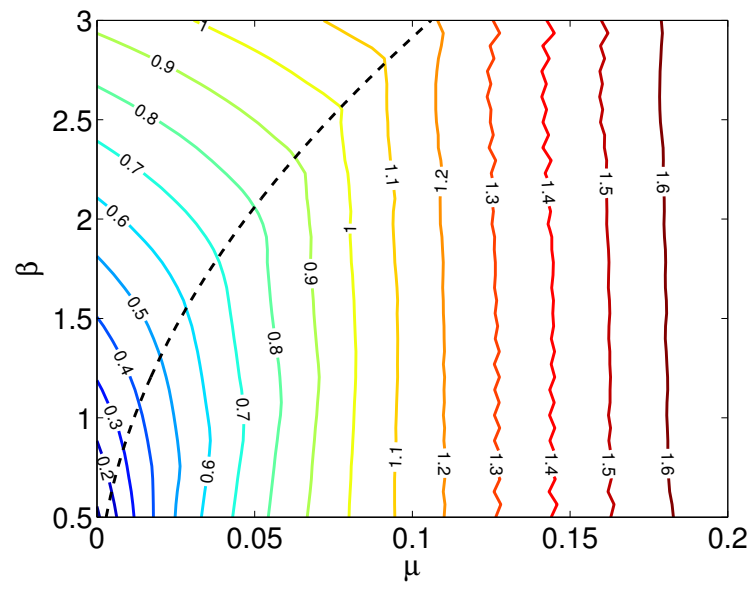

Figure 30: A contour plot of the average front speed of a $(1,2,3)$ hexagonal pattern on a cylinder which has its radius adjusted according to the nonlinear correction of the energetically preferred wavenumber. On the right half of the plot, it is clear that the front is pulled, since the speed does not depend significantly on $\beta$. On the left of the dashed line, the front is pushed, and on the right of the dashed line, the front is pulled. 


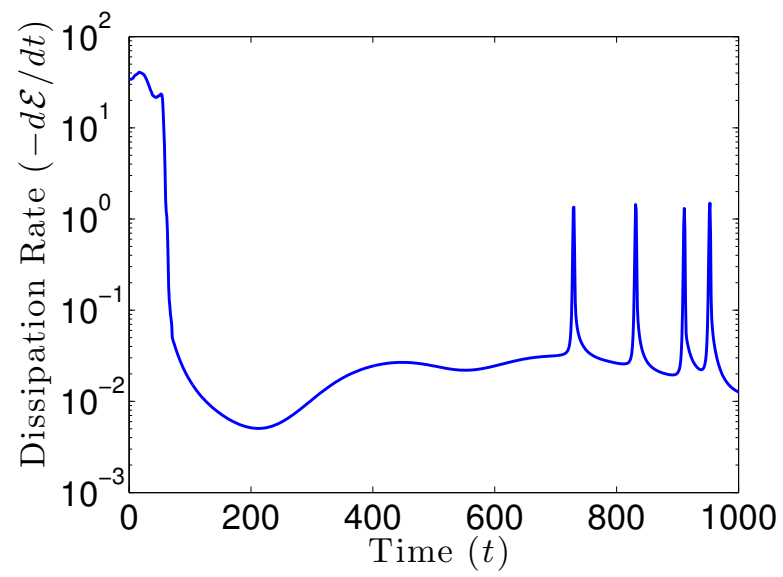

Figure 31: The long-time dissipation rate on the annulus $34<r<55$. The initially large value is due to the propagation of the pattern-forming front into the annulus, and the later peaks are due to the formation of defects. 


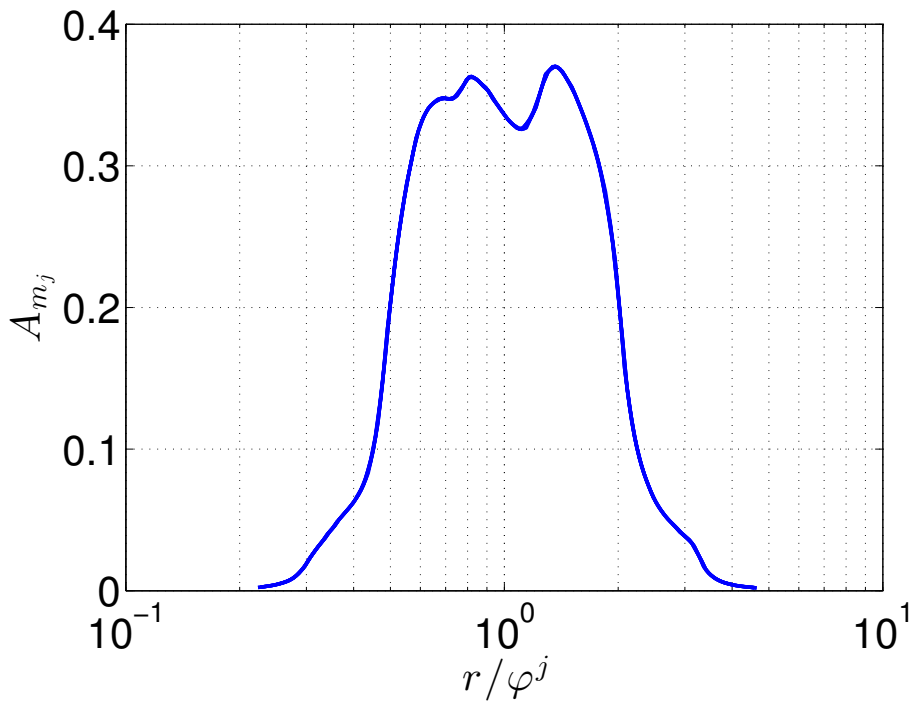

Figure 32: An invariant curve for the energy minimizing amplitudes $A_{m_{j}}$ with $\left\{m_{j}\right\}=\{1,2,3,5,8,13,21,34,55,89,144,233\}$. This has been computed by minimizing the energy over all amplitudes and wavenumbers on the locus of minima corresponding to the regular Fibonacci progression. The horizontal scaling emphasizes the self-similar property $A_{m_{j}}(r)=A_{m_{j+1}}(\varphi r)$. 


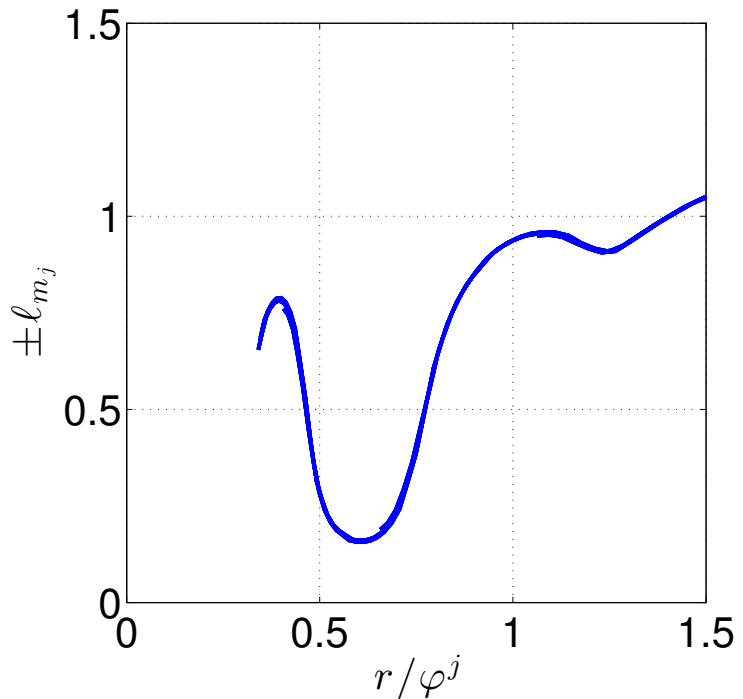

Figure 33: An invariant curve for the energy minimizing wavenumbers $\ell_{m_{j}}$ with $\left\{m_{j}\right\}=\{1,2,3,5,8,13,21,34,55,89,144,233\}$. This has been computed by minimizing the energy over all amplitudes and wavenumbers on the locus of minima corresponding to the regular Fibonacci progression. The horizontal scaling emphasizes the self-similar property $\ell_{m_{j}}(r)=-\ell_{m_{j+1}}(\varphi r)$. 


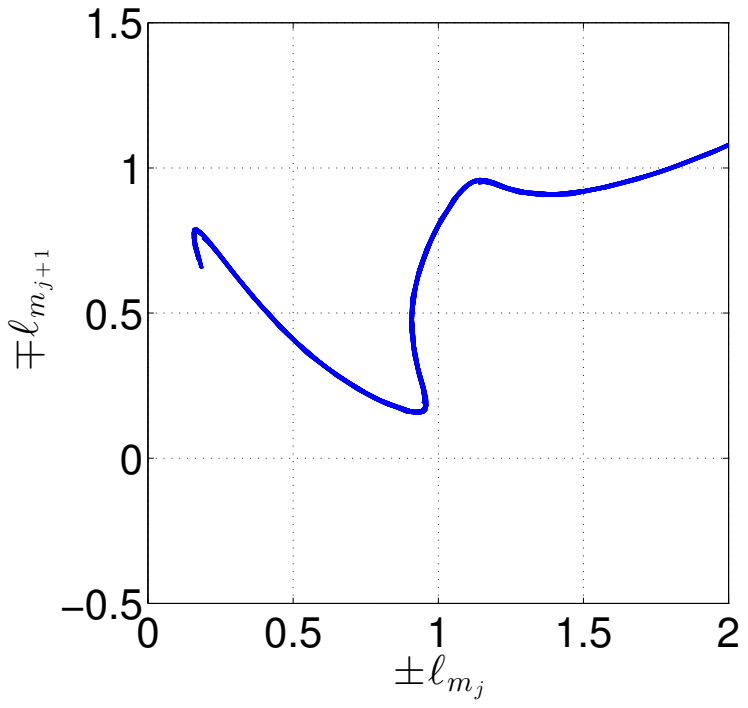

Figure 34: An invariant curve for the energy minimizing wavenumbers $\ell_{m_{j}}$ with $\left\{m_{j}\right\}=\{1,2,3,5,8,13,21,34,55,89,144,233\}$. This has been computed by minimizing the energy over all amplitudes and wavenumbers on the locus of minima corresponding to the regular Fibonacci progression. 


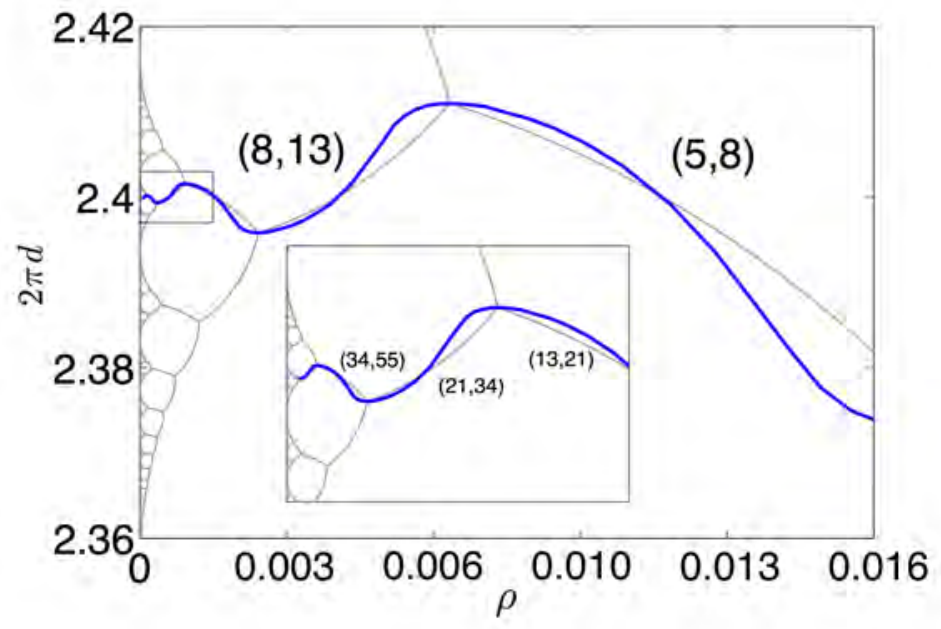

Figure 35: The divergence angle $2 \pi d$ and internodal spacing $\rho$ given by the wavevectors corresponding to the locus of energy minima of the amplitude equations along the main Fibonacci branches. Selected branches have been labeled with their parastichy numbers. 


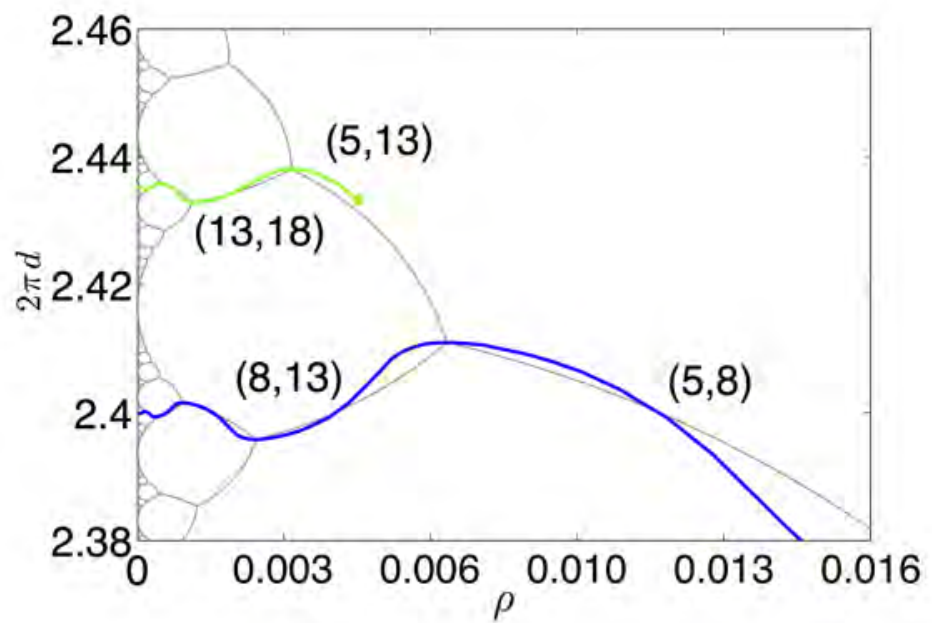

Figure 36: Again, the divergence angle $2 \pi d$ and internodal spacing $\rho$ given by the wavevectors corresponding to the locus of energy minima of the amplitude equations along the main Fibonacci branches. We also show an irregular Fibonacci branch, a new energy minimum corresponding to the selection of parastichy numbers $(5,13)$ rather than $(8,13)$ appearing at the end of the irregular locus. While $\rho$ decreases monotonically as $r$ increases on both branches, values of $r$ do not correspond to the same value of $\rho$ between branches. 


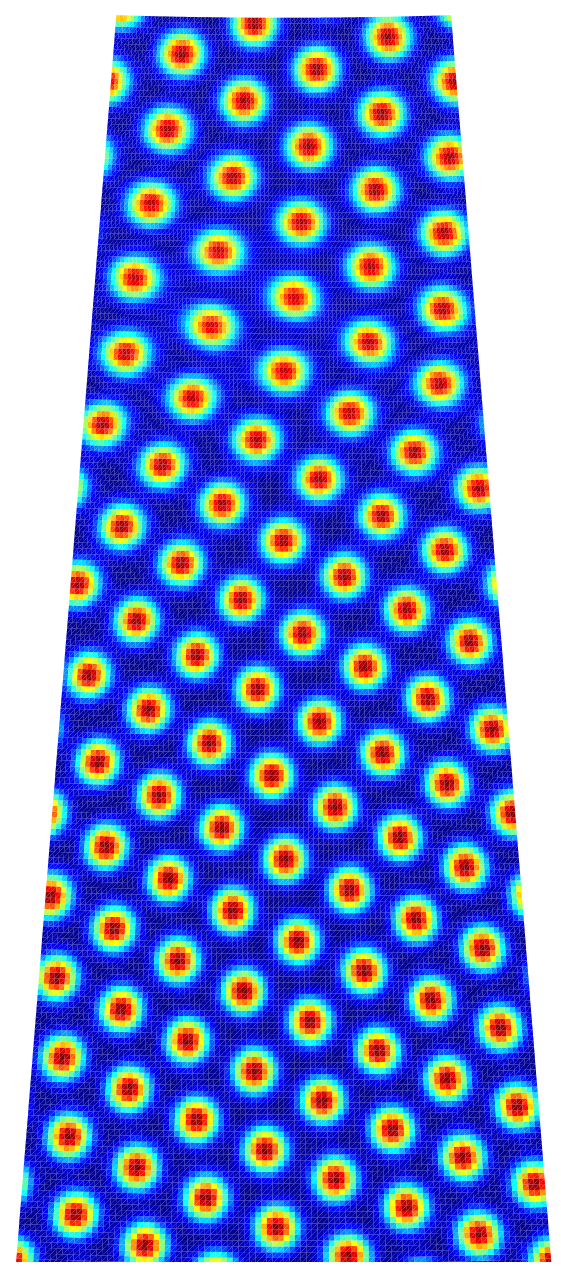

Figure 37: The pattern laid down by a front propagating on a frustrum, which has been cut and unrolled, starting at $r \approx 7.5262$ and decreasing to $r \approx 4.6866$ along a distance of 110 space units. It has been initiated as a $(3,5,8)$ hexagonal pattern and ends as a $(2,3,5)$ hexagonal pattern. This is an example of a type (II,2) transition. 


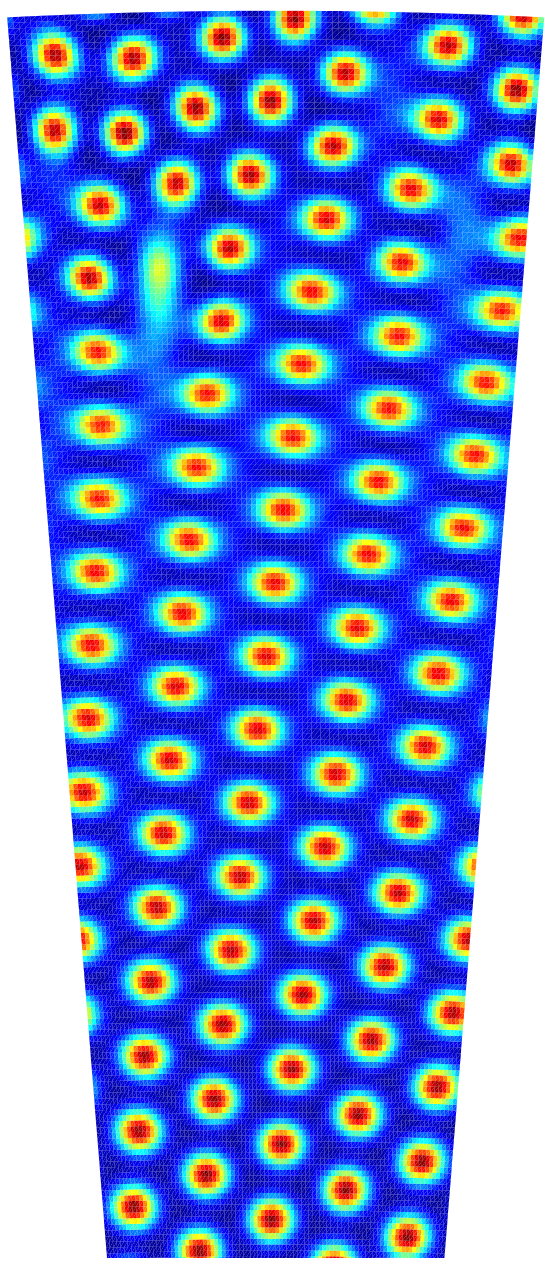

Figure 38: The pattern laid down by a front propagating on a frustrum, which has been cut and unrolled, starting at $r \approx 4.6866$ and increasing to $r \approx 7.5262$ along a distance of 110 space units. It has been initiated as a $(2,3,5)$ hexagonal pattern and ends as a $(3,5,8)$ hexagonal pattern, although it shares none of its wavevectors with the initial pattern. This is an example of a type $(\mathrm{I}, 0)$ transition. 


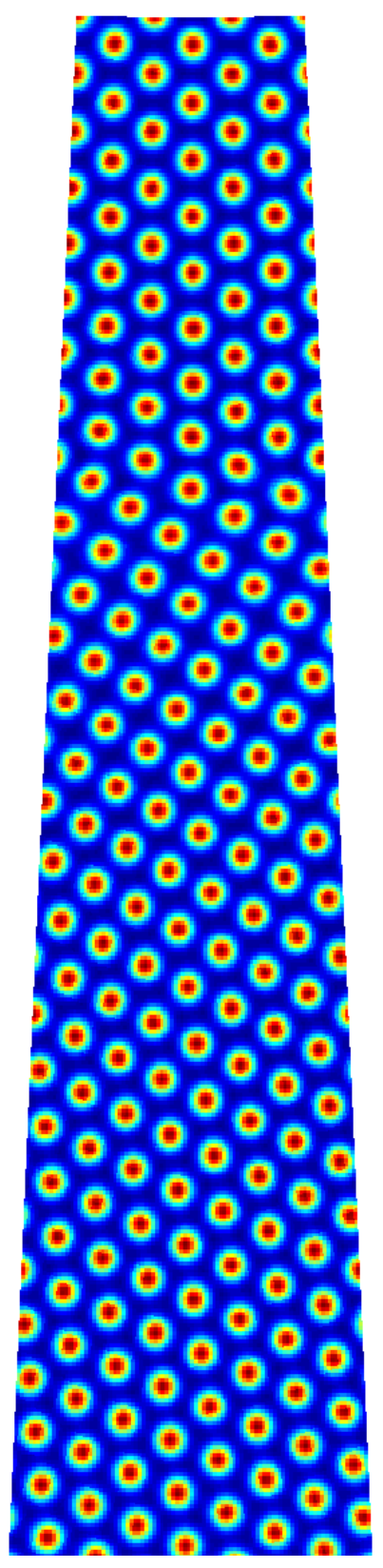

Figure 39: The pattern laid down by a front propagating on a frustrum, which has been cut and unrolled, starting at $r \approx 7.5262$ and decreasing to $r \approx 4.6866$ along a distance of 200 space units. It has been initiated as a $(3,5,8)$ hexagonal pattern and ends as a $(3,3,6)$ hexagonal pattern. This is an example of a type (I,1) transition. 


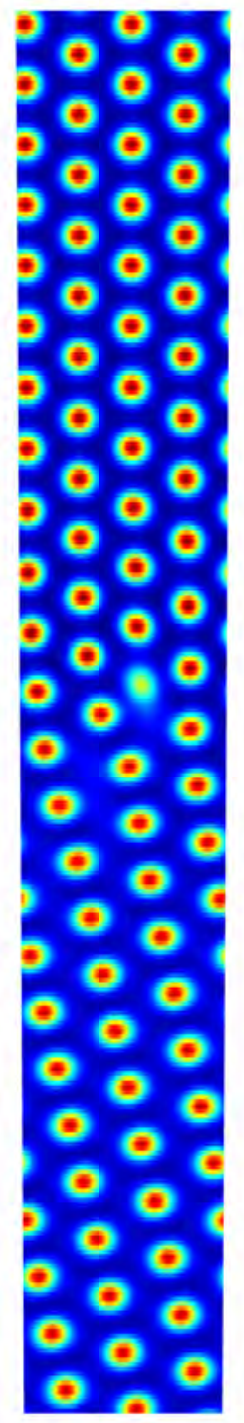

(a) A transition from a $(1,2,3)$ spiral planform at the bottom to a $(2,2,4)$ whorl planform at the top. This cylinder has $r \approx 3.54$.

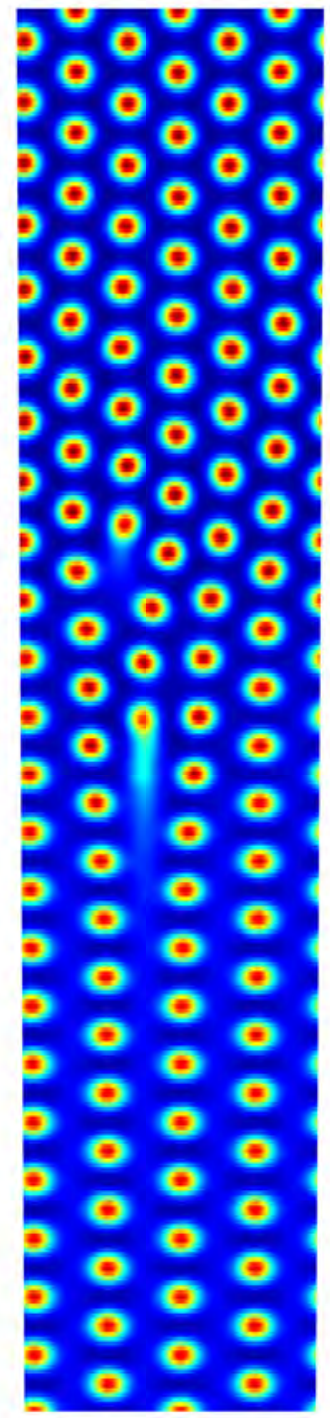

(b) A transition from a $(2,2,4)$ whorl planform at the bottom to a $(3,3,6)$ whorl planform at the top. Note the intermediate $(2,3,5)$ spiral planform. This cylinder has $r \approx 5.13$.

Figure 40: Illustrated here are two Type I, or discontinuous, transitions. 


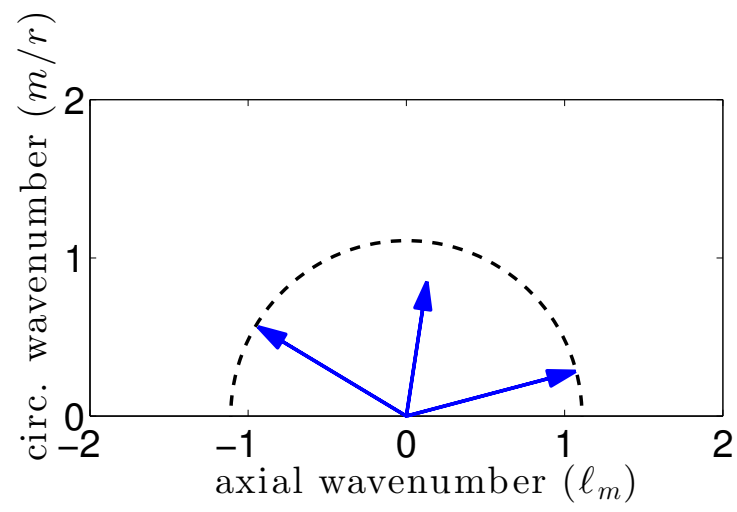

(a) The active wavevectors of the $(1,2,3)$ spiral planform. We denote these $\boldsymbol{k}_{2}, \boldsymbol{k}_{3}$, and $\boldsymbol{k}_{5}$ from left to right.

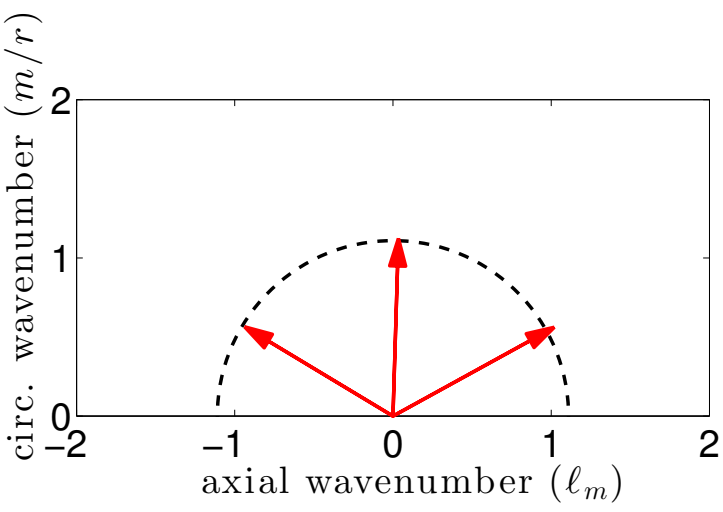

(b) The active wavevectors of the $(2,2,4)$ whorl planform. We denote these $\boldsymbol{k}_{2}^{-}, \boldsymbol{k}_{4}$, and $\boldsymbol{k}_{2}^{+}$from left to right.

Figure 41: Shown above are the active wavevectors before and after the transition illustrated in Figure 40a There are two important features to note: (1) It is clear that $\boldsymbol{k}_{2}=\boldsymbol{k}_{2}^{-}$. The $m=2$ wavevector remains unchanged through the transition. (2) There is a four-mode resonance $\boldsymbol{k}_{3}+\boldsymbol{k}_{2}^{+}=\boldsymbol{k}_{1}+\boldsymbol{k}_{4}$. 


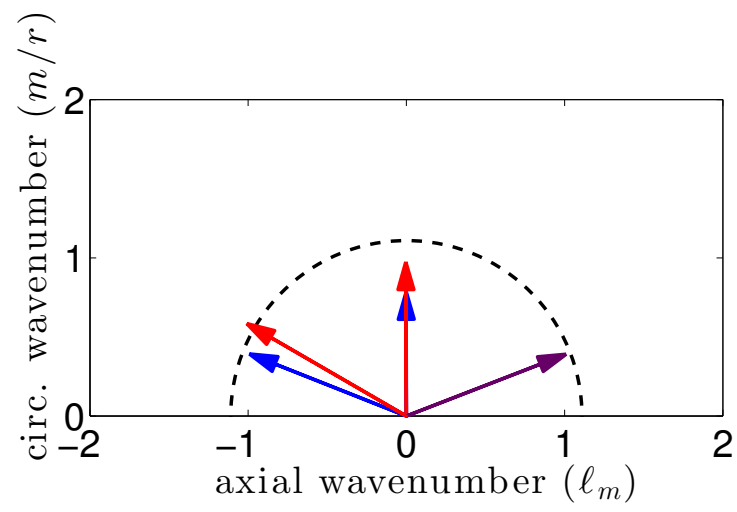

(a) The active wavevectors before and after the first intermediate transition from a $(2,2,4)$ whorl planform to a $(2,3,5)$ spiral planform. The rightmost wavevector is shared between the two planforms.

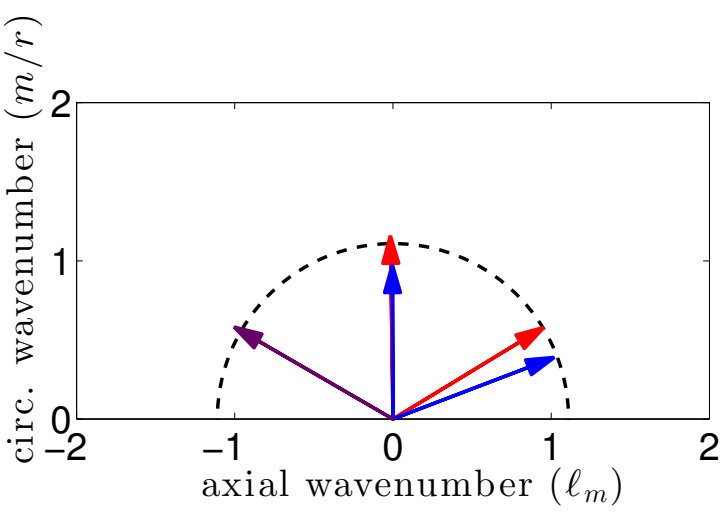

(b) The active wavevectors before and after the second intermediate transition from a $(2,3,5)$ spiral planform to a $(3,3,6)$ whorl planform. The leftmost wavevector is shared between the two planforms.

Figure 42: Shown above are the active wavevectors before and after each intermediate transition illustrated in Figure $40 \mathrm{~b}$ Overall, the transition begins with a $(2,2,4)$ whorl planform and ends with a $(3,3,6)$ whorl planform. 